EXPERIMENTAL TESTBED FOR ROBOT SKIN CHARACTERIZATION AND INTERACTION CONTROL

\author{
by
}

\author{
KYLE SHOOK \\ Presented to the Faculty of the Graduate School of \\ The University of Texas at Arlington in Partial Fulfillment \\ of the Requirements \\ for the Degree of
}

MASTER OF SCIENCE IN MECHANICAL ENGINEERING

THE UNIVERSITY OF TEXAS AT ARLINGTON

May 2014 
Copyright (C) by Kyle Shook 2014

All Rights Reserved 


\section{Acknowledgements}

First of all, thank you to my supervising professor Dr. Dan O. Popa for supporting me, challenging me and believing in me. The opportunity to work with him and the Next Generations Systems Group has been invaluable. I would also like to thank Dr. Woo Ho Lee for providing insight into my research along the way. I am grateful for Dr. Kamesh Subbarao and Dr. Haiying Huang for serving on my defense committee.

I would like to thank all of the NGS team for their support, especially Ahsan Habib and Cody Lundberg - without them I could not have completed this work. Thank you to General Lynch, Dr. Eileen Moss Clements, Dr. Muthu Wijesundara, Susan Craig, Priscila Walsh, Norm Spayd, Caleb Nothnagle, Stephen Savoie and everyone else at UTARI who has helped me along the way.

My utmost thanks and gratitude goes to my family. I could not write enough about how much they mean to me or how much they have all done for me throughout the years, so I won't even try. To my friends and other loved ones, thanks for sticking with me. To my brother Cole - let's roll!

This work was supported in part by National Science Foundation NRI Grant \#IIS1208623.

April 21, 2014 


\title{
Abstract \\ EXPERIMENTAL TESTBED FOR ROBOT SKIN CHARACTERIZATION AND INTERACTION CONTROL
}

\author{
Kyle Shook, M.S
}

The University of Texas at Arlington, 2014

\section{Supervising Professor: Dan O. Popa}

This thesis presents the research and work we conducted with an experimental testbed designed for testing and characterizing various types of robot skin with tactile sensing. The main goal of this research is to develop reduced-order models of robot skin for use in physical human-robot interaction simulations. The reduced-order models are developed from data collected by the testbed.

The experimental testbed presented is designed around a National Instruments compactRIO (cRIO). The cRIO controls and reads from a linear actuator and can collect synchronous measurements from multiple force sensors. The software developed for different test scenarios is also presented.

Experiments were conducted on force sensors, force sensors embedded in robot skin, and different materials for robot skin. Models built from these experiments are presented and validated through further testing. 
Table of Contents

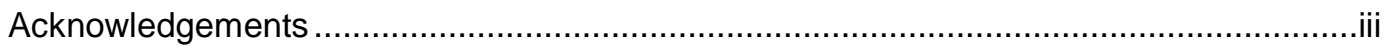

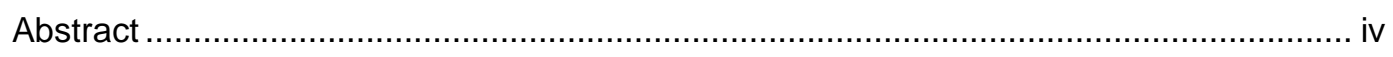

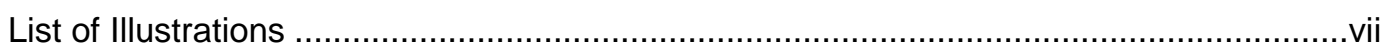

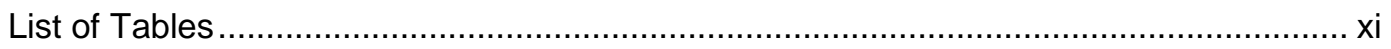

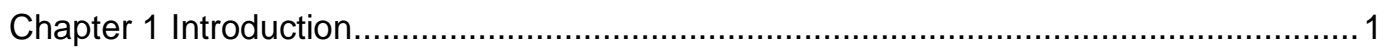

1.1 Motivation for Robot Skin Characterization .................................................. 1

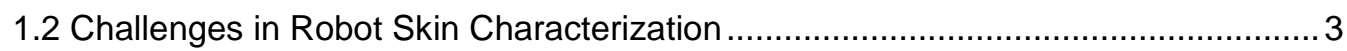

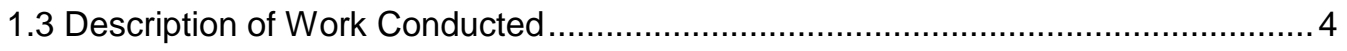

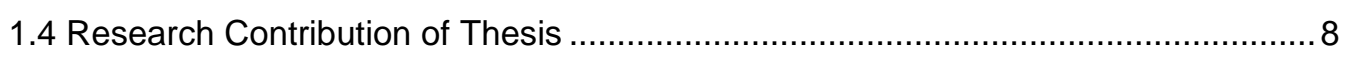

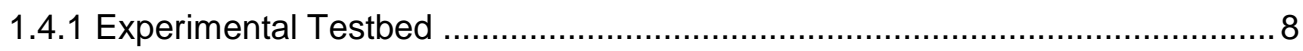

1.4.2 Modeling \& Characterization of Robot Skin \& Tactile Sensors ....................... 8

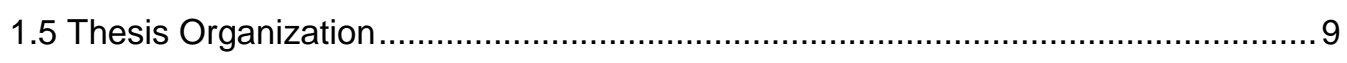

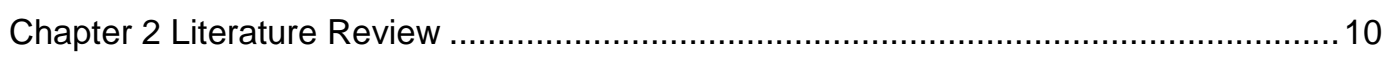

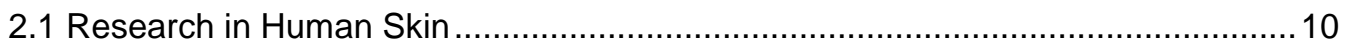

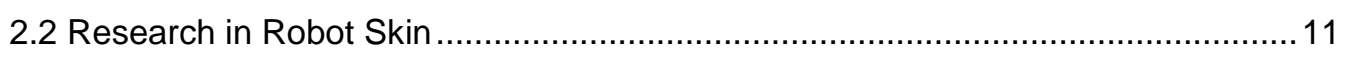

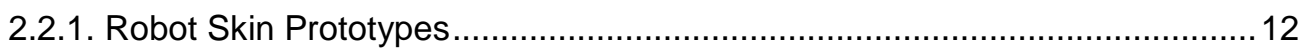

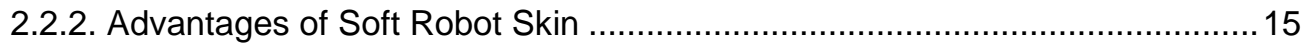

2.2.3. Robot Skin Manufacturing Technology ................................................ 16

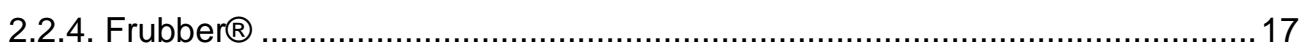

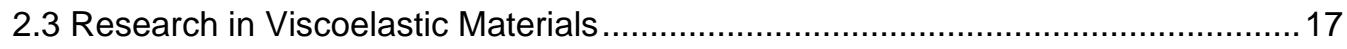

2.3.1. Linear Constitutive Models of Viscoelastic Behavior.................................. 18

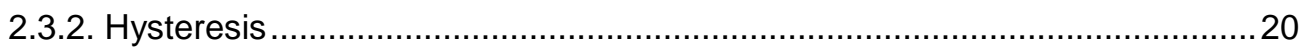

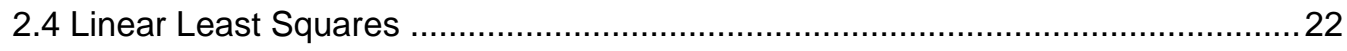

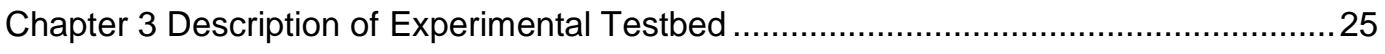




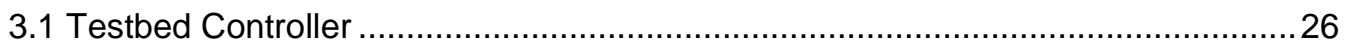

3.2 LabVIEW Motion Control \& Measurement Collection...........................................28

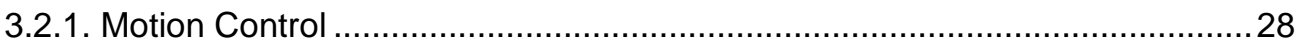

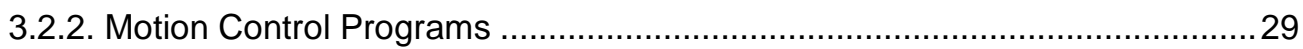

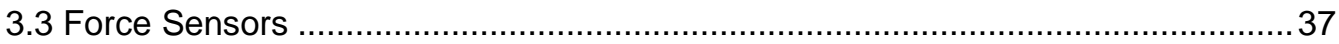

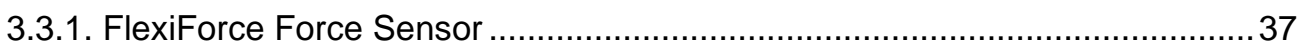

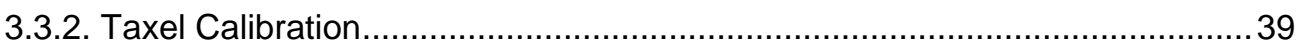

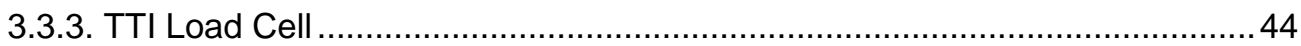

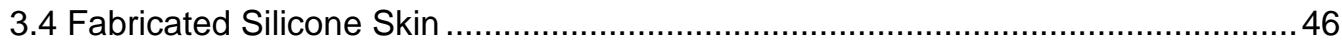

Chapter 4 Robot Skin Experiments \& Models ........................................................... 48

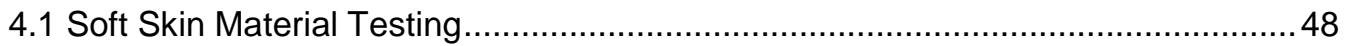

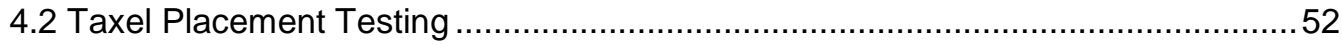

4.3 Robot Skin with Embedded Taxels Testing ................................................... 53

4.3.1 Parameter ID for Reduced-Order Models ...................................................53

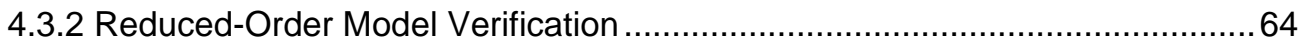

4.3.3 Effect of Soft Skin on Taxel Array ........................................................ 73

4.3.4 Taxel Loading Profile with Soft Skin............................................................ 75

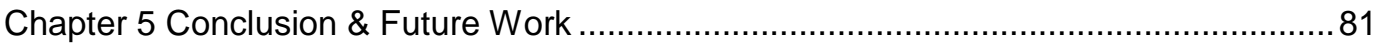

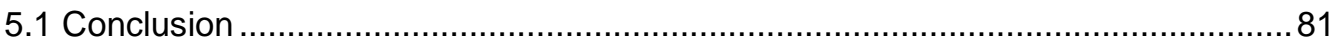

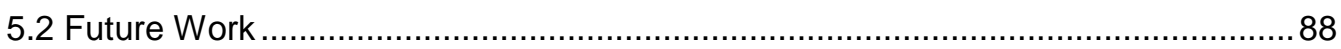

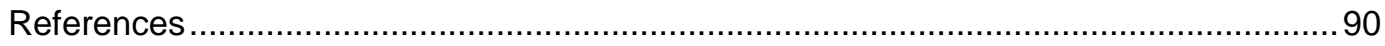

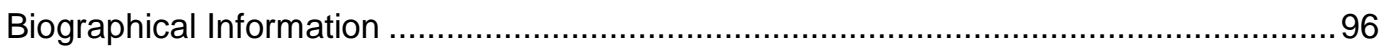


List of Illustrations

Figure 1-1 SkinSim user interface and sensor array on PR2 arm .............................. 2

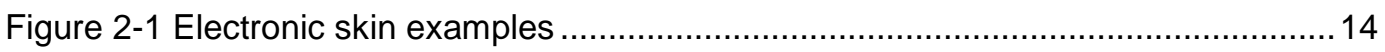

Figure 2-2 Behaviors of viscoelastic material ...................................................... 18

Figure 2-3 Rheological models of viscoelastic behavior ............................................ 19

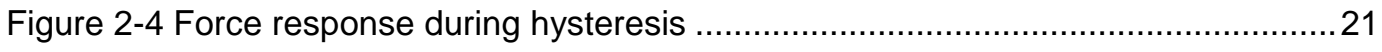

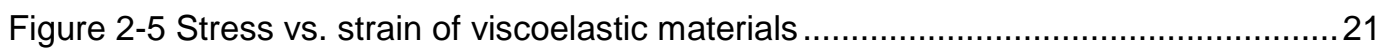

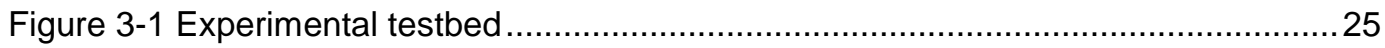

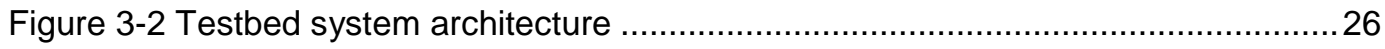

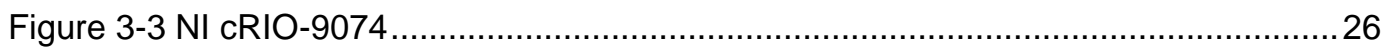

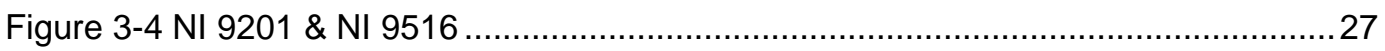

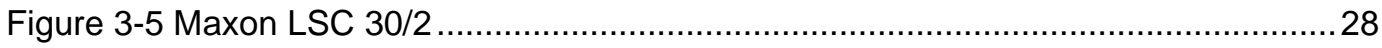

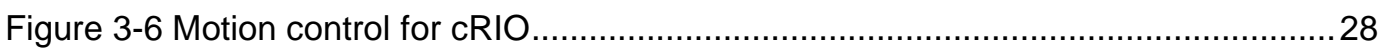

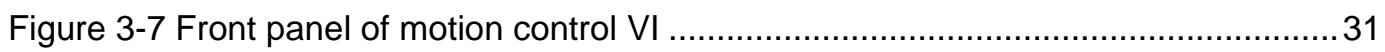

Figure 3-8 Timed-loop for measurement collection/storage ….......................................

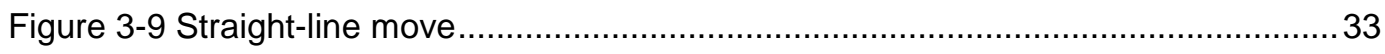

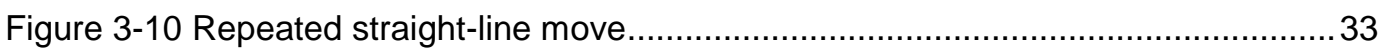

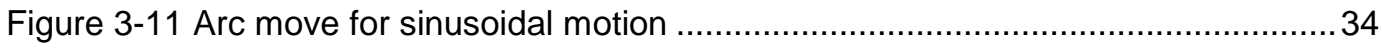

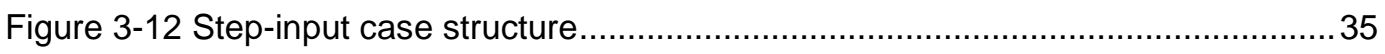

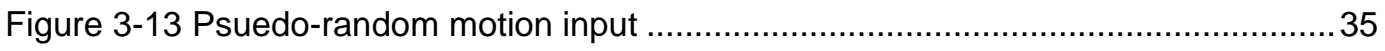

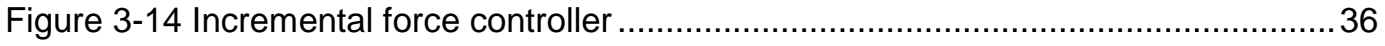

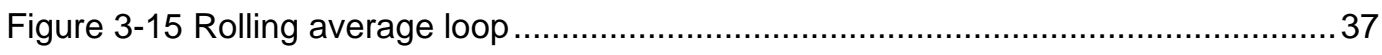

Figure 3-16 Tekscan FlexiForce A201 force sensor ................................................. 38

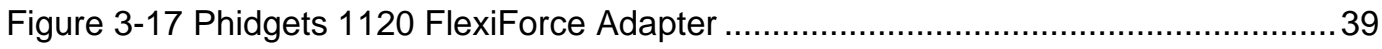

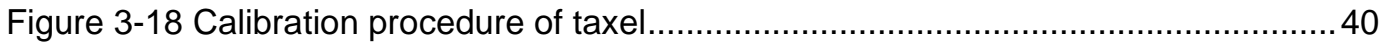


Figure 3-19 Taxel calibration fit

Figure 3-20 Calibration curve with hysteresis .......................................................... 42

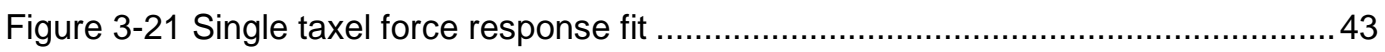

Figure 3-22 Single taxel force response hysteresis ............................................... 44

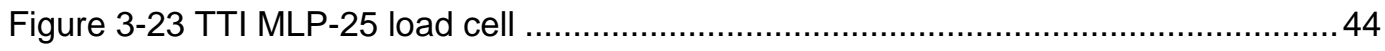

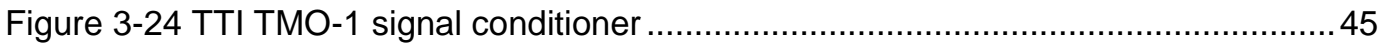

Figure 3-25 Mounting plate and load applicator ........................................................ 46

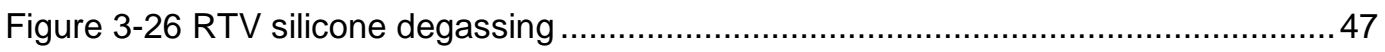

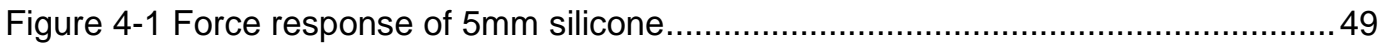

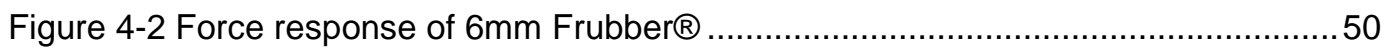

Figure 4-3 Stress relaxation of $5 \mathrm{~mm}$ silicone $\& 6 \mathrm{~mm}$

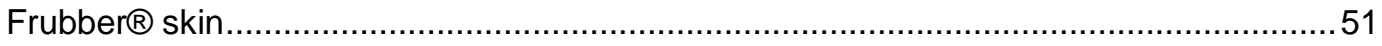

Figure 4-4 Force response of load cell \& taxel

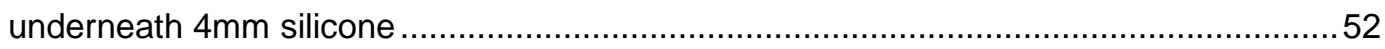

Figure 4-5 Force response of load cell \& taxel

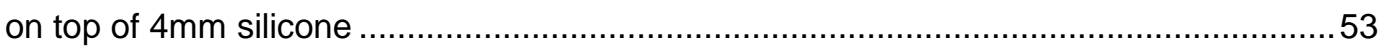

Figure 4-6 Cutout view of robot skin with embedded

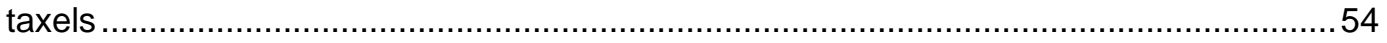

Figure 4-7 Force response of load cell \& taxel with

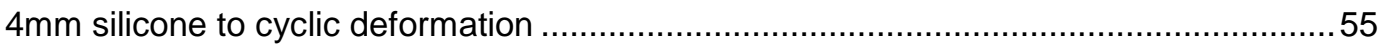

Figure 4-8 Force response of load cell \& taxel with

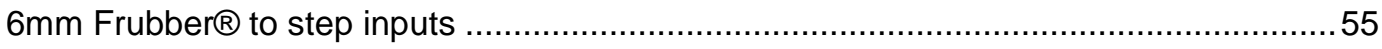

Figure 4-9 Reduced-order "dispersion" model relating applied force

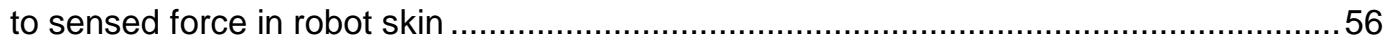


Figure 4-10 Sinusoidal motion measurements verses

calculated measurement model

Figure 4-11 Position error between measurements \&

model for $6 \mathrm{~mm}$ skin sample

Figure 4-12 Estimated values of $b_{1} \& b_{2}$ for adjusted

“dispersion" model.

Figure 4-13 Estimated values of $k_{1} \& k_{2}$ for adjusted

"dispersion" model.

Figure 4-14 Comparison of sensed load between measurements

\& "dispersion" model on $6 \mathrm{~mm}$ silicone skin

Figure 4-15 Taxel sensed force vs. "dispersion" model,

validation on $2 \mathrm{~mm}$ silicone skin

Figure 4-16 Taxel sensed force vs. "dispersion" model,

validation on $4 \mathrm{~mm}$ silicone skin

Figure 4-17 Taxel sensed force vs. "dispersion" model,

validation on $5 \mathrm{~mm}$ silicone skin

Figure 4-18 Taxel sensed force vs. LS identified "dispersion" model,

4mm Frubber® skin.

Figure 4-19 Load cell \& taxel response to sinusoidal deformation,

4mm Frubber® skin.

Figure 4-20 Comparison of taxel sensed force \& modeled force

using MATLAB's System Identification, 4mm Frubber ${ }^{\circledR}$ skin

Figure 4-21 Taxel sensed force vs. "dispersion" model, identified

with System Identification, validation on $4 \mathrm{~mm}$ Frubber ${ }^{\circledR}$ skin 73

Figure 4-22 Top view of 3x1 taxel array 74 
Figure 4-23 Overlapping sensing fields in 3x1 array

Figure 4-24 4mm silicone skin loading profile ........................................................ 79

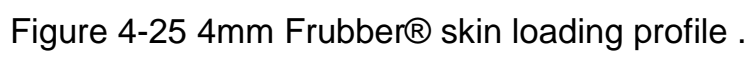
80 
Table 3-1 Slope values calculated from calibration curves of taxel voltage output vs. applied force.

Table 3-2 Slope values calculated from curves of taxel

deformation vs. applied force

Table 4-1 Average values of identified parameters of "dispersion"

model for $2 \mathrm{~mm}$ silicone skin samples

Table 4-2 Average values of identified parameters of "dispersion"

model for $4 \mathrm{~mm}$ silicone skin samples

Table 4-3 Average values of identified parameters of "dispersion"

model for $6 \mathrm{~mm}$ silicone skin samples

60

Table 4-4 Average values of identified parameters of "dispersion"

model for each thickness of silicone skin

Table 4-5 Average values of identified parameters of adjusted

"dispersion" model for each thickness of silicone skin

Table 4-6 Average values of identified parameters of adjusted

"dispersion" model for 4mm Frubber ${ }^{\circledR}$ skin

Table 4-7 Identified parameters for "dipersion" model using

MATLAB's System Identification, 4mm Frubber $®$ skin

Table 4-8 Estimated values of general Gaussian model with $4 \mathrm{~mm}$

silcone skin, also $\mathrm{R}^{2}$ value

Table 4-9 Estimated values of general Gaussian model with 4mm

Frubber ${ }^{\circledR}$ skin, also $\mathrm{R}^{2}$ value

Table 4-10 Taxel response $(\mathrm{N})$ for varying deformation with $4 \mathrm{~mm}$

silicone skin 
Table 4-11 Taxel response $(\mathrm{N})$ for varying deformation with $4 \mathrm{~mm}$

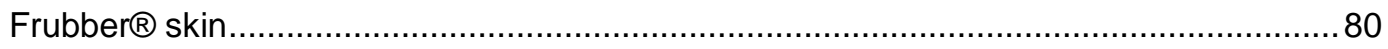


Chapter 1

Introduction

\subsection{Motivation for Robot Skin Characterization}

Generally speaking, robots are able to reliably perform repeated, specific tasks when their movements are predefined and reside in structured environments, for instance in manufacturing. Servo technology and control enables manufacturing robots to far exceed any human when comparing the two in precision, accuracy and repeatability of movements, yet place the most advanced robot in a random kitchen and it would be much more difficult for it to fetch a glass of water [1]. One of the reasons for this is an increasing need for robots to perceive their surroundings to the extent that humans can.

Robot skin with embedded multi-modal sensors, such as temperature, pressure, and proximity is one direction that has been pursued for several decades. For our purposes we will classify such robot skin as a "heteroceptive sensor inspired by nature that could eventually enable social robots to share their workspace with humans" [2]. This defines robot skin as transmitting information through multiple modalities, with human-like qualities (aesthetically and in transmission, control, etc.) and enabling a situation where humans can safely go about their business in proximity to the robot without the need for structured barriers (real or imaginary).

Electronic sensors are the primary method for robots to gather information about their surroundings. There are many types of sensors that have been exploited in robotics already, including vision, pressure, vibration, temperature, proximity, touch and infrared, and have been routinely used to reduce uncertainty for robots in an unknown environment [1]. Pressure, vibration, temperature and touch fall in the category of tactile sensing, or sensing through physical interaction, which allows the robot to discern the 
physical nature of the objects or humans in near proximity. In a sense, tactile perception allows humans to differentiate between what is "me" and what is "not me" [3].
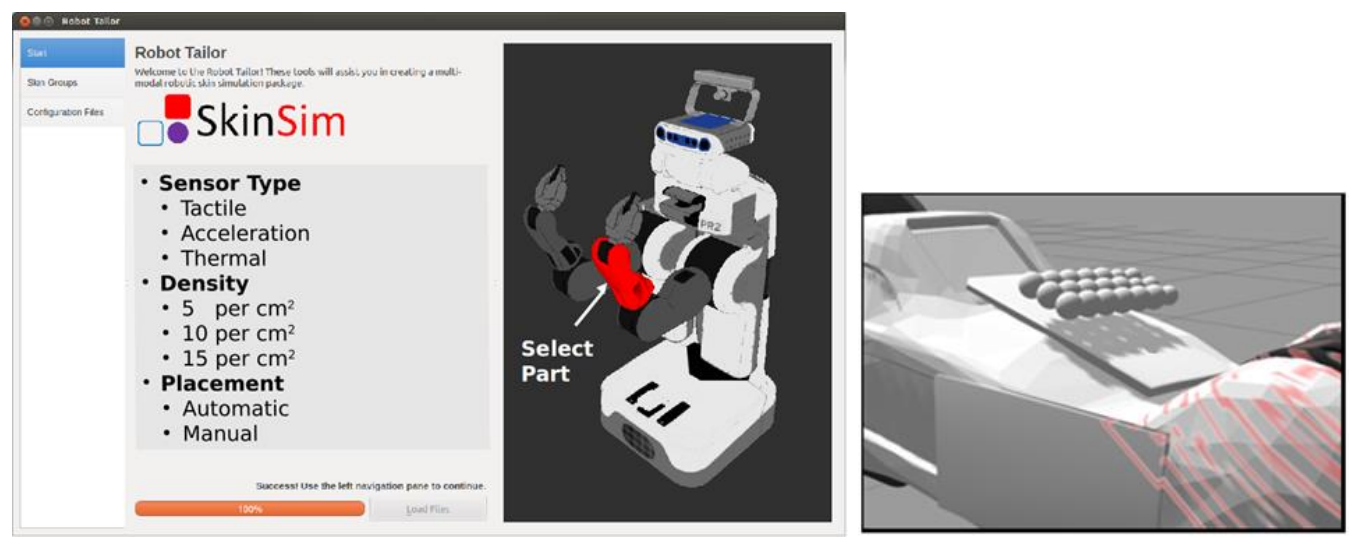

Figure 1-1 SkinSim user interface and sensor array on PR2 forearm [4]

Tactile sensing and tactile transduction methods have been studied extensively as discussed in chapter 2. However, there is limited prior research on design tools for tactile skin that considers questions of optimal sensor placement, density, resolution, bandwidth, even modality [3]. Realistic robot skin system models can be very helpful in reducing trial and error, and avoiding costly and time consuming design iterations for robot skin. Toward this aim, a skin simulation environment - SkinSim - is currently under development at the University of Texas at Arlington (UTA) in the Next Generation Systems Group (NGS). SkinSim is a simulator framework for multi-modal robot skin as a physical interface between robots and humans [4]. SkinSim will be used for design optimization of robot skin parameters using real time feedback and can test methods for optimal placement, data management and types of sensors. In SkinSim realistic reducedorder models of robot skin must be implemented to relate external forces applied to the skin to force measurements gathered on taxels embedded in the skin. Reduced-order models of robot skin will also be used in simulated force control experiments. This includes but is not limited to creating models of individual sensors, sensor arrays, 
sensors embedded in robot skin material and the robot skin material itself. This work discusses methods to develop reduced-order models that can and will be used in SkinSim.

\subsection{Challenges in Robot Skin Characterization}

Biologically inspired technology looks to the oldest form of optimization for clues on how to improve our present day technology. If we look to human skin in this manner, we will find that tactile sensing for humans is "[hysteretic], nonlinear, time varying, and slow" [3]. None of those qualities sound like ways we would like to describe our latest expensive robot skin. Fortunately for humans, we possess a central nervous system that can derive useful information from our own "technologically poor" sensors [3].

Unfortunately, robots have nothing analogous in scope with that yet. Biological inspiration aside, many other challenges are present in robot skin characterization.

Two of the main forms of tactile transduction, piezoresistive and capacitive, involve deforming materials to measure a change in resistance or capacitance, respectively. All deformable materials experience some degree of hysteresis. Hysteresis is a nonlinear phenomenon where the relationship between a system's input and output

differ between loading and unloading [5]. Another issue piezoresistive and capacitive sensors experience is drift. Drift, like hysteresis, is a nonlinear behavior where input stimuli on materials require a recovery time to produce an equivalent output. Model parameters for both hysteretic and drift behavior can be identified for individual materials/sensors for use in a simulation environment. The challenge can be in selecting what model to use for what behavior and material. Outside of parameter identification, there can be challenges implementing these models because of their computation requirement and memory storage of previous states. 
Tactile sensors experience unit-to-unit variation between sensors of the same type and brand. This can be remedied through calibration of each individual sensor unit. Calibration procedures must be consistent for each unit to compare signal output from multiple sensors.

With many types of tactile sensors, a dynamic testbed must be designed to accommodate them. The testbed must record synchronous measurements from multiple instruments including tactile sensors, motion control devices and other sensors used for characterization.

Model identification requires prior wisdom on the system in question. Fitting experimental data to randomly selected models is not an efficient method of model identification. Model components need to be based off of the physical phenomena they are describing. Randomly selected model components with no meaning should not increase the soundness of a model. An example is viscoelastic material; the elasticity of the material can be modeled by a spring, the viscous behavior by a dashpot.

\subsection{Description of Work Conducted}

In this thesis we present the experimental testbed used to perform experiments on tactile sensors. We also present the methods used to analyze the measurements gathered from these experiments that help characterize different setups of robot tactile skin. Reduced-order models were created for three different thicknesses of robot skin using piezoresistive pressure sensors.

The experimental setup was created with equipment from the UTA Research Institute (UTARI) and NGS labs. A linear actuator with encoder feedback was mounted vertically on an optical table above a horizontal $x-y$ stage. The linear actuator was controlled by a National Instruments (NI) compactRIO (cRIO). The cRIO was 
interfaced with a NI servo-drive module and a Maxon servo amplifier through a terminal block and custom cabling to communicate with the linear actuator.

The purpose of the linear actuator was to control the movement of a cylindrical plunger as it depressed into different types of robot skin and tactile sensors. Force and pressure data from the plunger were recorded through an attached in-line load cell. We designed and printed, with a rapid prototyping machine, a mounting plate to attach the load cell to the linear actuator. The mounting plate was designed so that forces from the plunger would produce minimal torqueing on the linear actuator axis.

LabVIEW (LV) programs were created that controlled the motion of the plunger mounted on the linear actuator and recorded time-stamped measurements from the load cell, tactile sensors and encoder. The programs were run from the cRIO using Scan Interface mode. The cRIO Scan Interface mode is optimal for sampling rates up to $300 \mathrm{~Hz}$ [6]. Sampling rates above $300 \mathrm{~Hz}$ should utilize FPGA control. The sampling rate was $100 \mathrm{~Hz}$ during all of our testing. Programs were written for the following experiments:

- $\quad$ Single or repeated step input loading

- $\quad$ Stair-step loading/unloading

- $\quad$ Random step input loading/unloading

- $\quad$ Sinusoidal loading/unloading

- Incremental force control

Program parameters were set on the LV user interface (UI) and then compiled and ran from the $\mathrm{cRIO}$ internal processor. Live measurements were visible on the UI but were set to update at a slower rate than the measurement collection rate of $100 \mathrm{~Hz}$. Inside the LV programs priority levels can be set for separate timing loops; so for our purposes, the measurement collection and motion control loops were highest priority, followed by the UI update loop at a significantly lower priority. This ensures the measurement 
collection and motion control takes precedence over updating the UI. Once the experiment was completed the measurement data was extracted from the $\mathrm{cRIO}$ memory. Measurements from the load cell and tactile sensors were amplified through separate signal conditioners and sent to an analog input module on the cRIO. The piezoresistive tactile sensors we chose, FlexiForce taxels from Tekscan, were tested for drift and individually calibrated. The force applied was the load measured by the load cell; the force sensed was the load measured by the calibrated taxel. A total of four FlexiForce sensors were tested.

Silicone skin samples were fabricated from molds in thicknesses of $2 \mathrm{~mm}, 4 \mathrm{~mm}$ and $6 \mathrm{~mm}$. This required printing of square molds in a 3D rapid prototyping machine. To improve the removal of the silicone from the mold cavities all of the molds were coated with an ultrathin layer of parylene by a SCS parylene deposition system. The silicone used for the skin samples were room temperature vulcanizing (RTV) silicone rubber mixed in a 10:1, base to curing-agent ratio. Once the mixture is poured into the mold cavities they undergo degassing to remove air bubbles. After degassing the samples are oven cured and removed from the molds. Three different samples were fabricated at each thickness. All of the samples were not mixed at the same time and not all air bubbles were removed so each sample is not homogeneous. Instead, each sample has slightly different properties.

Taxels were fixed to the $x-y$ stage and centered under the plunger. Once centered, the skin samples were taped to the taxel surface and pressed down on to ensure the bottom of the samples were flat against the taxel and stage surface. To identify parameters for reduced-order models representing robot skin, a sinusoidal input was provided as stimulus to the skin surface. We tested three thicknesses of silicone skin $(2 \mathrm{~mm}, 4 \mathrm{~mm}$ and $6 \mathrm{~mm})$ and one sample of Frubber ${ }^{\circledR}$ skin. The silicone skin deformation 
was fitted to a sinusoidal curve that was then used, along with the taxel and load cell force measurements, in a least squares algorithm to identify the parameters for the reduced-order model. With the model it is possible to relate the applied load on robot skin to the sensed load of a taxel embedded in the skin. Three verification tests were performed on silicone skin verifying the effectiveness of the reduced-order model to mimic the output of the taxel. The same reduced-order model was used with the $4 \mathrm{~mm}$ Frubber ${ }^{\circledR}$, however, parameter identification for the model was performed using a transfer function identification method from MATLAB's System Identification Toolbox instead of a LS algorithm.

A larger mold of $4 \mathrm{~mm}$ thickness was fabricated for use in experiments with a taxel array. Three taxels were fixed side by side to the $x-y$ stage forming a $3 \times 1$ array. The taxel array was then covered with the skin sample. The load applicator was depressed into the skin multiple times along the length of the taxel array in defined increments. The test was designed to understand the transmission of force that occurs through robot skin to adjacent taxels that are not experiencing a direct perpendicular application of force. A loading profile was created from the sensor measurements from this test for each skin sample. A similar experiment was performed on a $4 \mathrm{~mm}$ silicone and $4 \mathrm{~mm}$ Frubber ${ }^{\circledR}$ sample to model the load distribution at distances a certain distance from the taxel center. From the measurements a Gaussian distribution was fit for both silicone and Frubber® skin.

Experiments were conducted to highlight the viscoelastic behavior of two types of soft robot skin. First, repeated step loading and unloading was applied to silicone rubber and Frubber®. Force measurements collected from the load cell show varying degrees of hysteresis, a nonlinear behavior, for each sample. Second, a constant strain input was applied to each sample. Measurements from the load cell make it clear that drift is 
another nonlinear problem encountered when modeling robot skin. In the future, nonlinear models to mimic the hysteresis and drift behavior will be implemented for improved interaction control in SkinSim.

An experiment was conducted testing the difference between a taxel placed on top of robot skin versus underneath. The same step loading and unloading was applied to a $4 \mathrm{~mm}$ skin sample with the taxel fixed to the bottom of the $x-y$ stage and the taxel taped to the top of the skin sample.

\subsection{Research Contributions of Thesis}

\subsubsection{Experimental Testbed}

A primary contribution of this thesis is the experimental testbed. The testbed is designed for multipurpose motion experiments with a focus on robot skin testing and characterization. Experiments on the testbed are capable of applying predefined motion profiles on robot skin and tactile sensors while sending and recording signals from an analog input module, an analog output module and a digital I/O module. Multiple LV programs were written to control the motion of the linear actuator as well as an incremental force controller.

\subsubsection{Modeling \& Characterization of Robot Skin \& Tactile Sensors}

Experiments were conducted to better understand how the thickness of robot skin affects the transmission of applied force to a taxel directly underneath the contact point and to taxels adjacent to the point of contact. The placement of taxels on top of or underneath robot skin was also looked at.

Reduced order models were identified for varying thicknesses of silicone and Frubber ${ }^{\circledR}$ robot skin for use in the sensor optimization program SkinSim. These scalable models will allow further tests to be performed in a simulation environment to determine optimal parameters for robot sensing like taxel placement, taxel density, etc. 
In addition to the reduced-order models, Gaussian fits of the load distribution through silicone and Frubber ${ }^{\circledR}$ skin were identified. The Gaussian fits can be used in defining load profiles throughout a large array of sensorized skin in SkinSim.

\subsection{Thesis Organization}

Chapter 2 is a literature review in the following: 1) research in human skin properties and tactile signal processing in humans; 2) successful implantation of wholebody robot skin over the last two decades and advanced technology in robot skin; 3) research in viscoelastic materials; 4) parameter estimation with linear least squares.

Chapter 3 begins with a detailed description of the experimental testbed. The cRIO and LV programs are discussed along with the sensors and accompanying components used in our experiments. Results from the calibration of four FlexiForce taxels are presented. Additionally, fabrication of silicone skin is discussed.

Chapter 4 outlines the data collection and insights of our experiments on robot skin. First we look at drift and hysteresis of silicone skin and Frubber® and the challenges they present. Then we describe the effects of taxel placement and the transmission of force picked up by a sensor array. Finally we present a reduced-order model for robot skin relating the applied force on the skin to the sensed force by a taxel in the skin. We describe the methods for gathering data for parameter identification and present three validation cases.

Chapter 5 concludes the thesis by discussing the results presented and provides an outline for future research that could build upon our work. 
Chapter 2

Literature Review

\subsection{Research in Human Skin}

Along with sensing technology, another facet of robot skin that is in constant development is the skin-like covering used to house and protect sensors on social robots. The Uncanny Valley has been studied at length and has proven to be an important factor in the acceptance of social robots [7]. While it may be unnecessary for robot skin to exactly mimic human skin, it could be important for robot skin to be "skin-like" at the least. To better develop human-like robot skin, it is first important to understand human skin.

Human skin exhibits a nonlinear stress-strain relationship and ultimate elongation of $74-96 \%$ on the legs and arms [8]. It is "multilayered..., nonhomogeneous, and viscoelastic" [3]. Fingertip skin conforms to objects during grasping, spreading out the detection to multiple mechanoreceptors, and contains intermediate and papillary ridges that improve grip [3].

Human skin consists of multiple sensing modalities including temperature, pressure, vibration and pain. These "sensors" in human skin have varying density over different parts of the human body. For example, mechanoreceptors in human hands range in density from 241 per square centimeter in the fingertips to 58 per square centimeter in the palms [9]. Receptor density relates to spatial acuity. Two point discrimination acuity in the fingers is less than $1 \mathrm{~mm}$ and greater than $30 \mathrm{~mm}$ in the forearm, back and calf [10]. The mean pressure sensitivity threshold in female and male hands is approximately $2.5 \mathrm{mg}$ and $3 \mathrm{mg}$, respectively; mean temperature thresholds for both sexes of warm and cold is approximately 38degC and 32degC, respectively [11]. Human skin can detect vibrations up to a frequency of $700 \mathrm{~Hz}$ and can distinguish 
between consecutive collisions in the fingertips at different locations down to $30-50 \mathrm{~ms}$ [12] [13].

Research in [3] details "hints" for designing robot skin that is modeled after human skin. They call for multi-modal, distributed skin with a resolution mimicking that of human skin, e.g., higher resolution in areas used for grasping and lower for areas that will receive less contact. They also call for sensors with high sensitivity, dynamic range and quick response.

In addition to physical properties of robot skin and sensors, we can also look to the human body for influence and inspiration on the control and processing of information from sensors. In humans, signals from mechanoreceptors are transferred through nerves in the form of action potentials which is "similar to digitizing and coding analog signals by an analog-to-digital convertor" [3] [14]. The signals from mechanoreceptors then travel through multiple paths and at different speeds depending on the level of importance and also undergo preprocessing before the signal reaches the central nervous system [3]. This helps streamline information processing and reduces the "load" on the bodies' main "processor". This can be replicated in robot skin through sensing units with local processing.

\subsection{Research in Robot Skin}

Tactile sensing technology in general has been widely reviewed [15] including tactile sensing for dexterous manipulation by robots [16]. While specific components behind robot skin technology have been researched for half a century, only in the last two decades has whole-body robot sensing been fully investigated. In 2001, Lumelsky, a pioneer in robot skin, proposed flexible, large-area, "sensitive skin" for robots composed of multi-modal sensors to allow robots to interact with humans in an unstructured environment [17]. Much progress has occurred in robot skin technology since Lumelsky's 
proposal: from improvements in manufacturing flexible printed circuit boards (PCB) [18] [19] to multi-sensing units deployed on functioning social robots [20]. In this section we will discuss research in robot skin, from Lumelsky's proposal to today's achievements.

\subsubsection{Robot Skin Prototypes}

Lumelsky demonstrated the possible elegance of humans and robots interacting with a ballerina safely dancing with a sensorized robot arm [17]. The arm's surface was covered with infrared sensing taxels that detected the nearby presence of humans and other objects. The sensors prevented the arm from colliding with and damaging its "partner", an absolute requirement for any pHRI. Unique to Lumelsky's research was the idea that the entire surface of the robot must be covered in "sensitive" skin. "Sensitive", in this case, refers to the ability to detect one's local surroundings through multiple modalities: proximity, temperature, pressure, touch and others. Lumelsky also discussed the massive amount of processing power that would be required to handle the data from the number of sensors it would require to sensorize an entire robot. He foresaw this problem, the immense density of data and sensors, as the main restriction for robot sensing moving forward. However, he also discussed that once this hurdle of massive parallel information processing is tackled, the knowledge learned from the technology would be applied in countless fields in new and currently unknowable ways.

HEX-O-SKIN, a project from Germany, is a self-organizing, multi-modal sensing module that has been deployed on a KUKA arm and HRP-2 robot [20] [21]. The module has four sensing modalities: proximity, acceleration, temperature and normal force. The proximity, acceleration and temperature sensors are commercial off-the-shelf (COTS) while the normal force sensor is custom made. The module is a hexagon with sides $1.4 \mathrm{~cm}$ in length and has a 3D printed elastomer cover. Each module has local intelligence and a unique ID that allows it to determine where it is in relation with the 
other interconnected modules. This ability, along with the accelerometer, allows the HEXO-SKIN modules to create a map of their location relative to the robot host. To add robustness to the sensing system it incorporates a "highly redundant network structure". Of the four ports located on each module, it is only necessary that one is working at any given time. This is possible because the modules can reroute information through other adjacent modules if they detect a broken connection. Grasping experiments of large objects, a trashcan, were performed with the modules incorporated on a HRP-2 robot. The HRP-2 used feedback from "tactile driven motion primitives" to adjust its arms to grasping the object.

Another example of a fully-developed robot skin employed on iCub, KASPER and NAO robots is an Italian project called ROBOSKIN [22]. Like HEX-O-SKIN, ROBOSKIN consists of small, interconnected PCB sensing modules. Unlike, HEX-OSKIN, ROBOSKIN limits its sensing to one modality, capacitive pressure sensing, and the modules are flexible. Each triangular module, sides $3 \mathrm{~cm}$ in length, is composed of twelve individual capacitive taxels, with up to sixteen triangles able to connect and communicate. Each PCB module incorporates local processing with a COTS analog-todigital convertor. Custom mounting components were created to incorporate ROBOSKIN on each robot. Once embedded on the robot surface, the modules were covered in silicone foam. After experiencing problems with relevant drift, two of the twelve taxels were made to respond to changes in temperature [23]. These two taxels were then used to compensate for temperature variation in the other ten taxels. On the iCub, modules were implanted in the forearms, palm and fingertips. Grasping experiments were performed demonstrating the improved ability of the iCub to pick up deformable objects with tactile feedback versus without. 
(a)

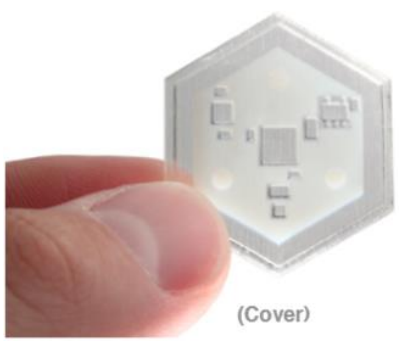

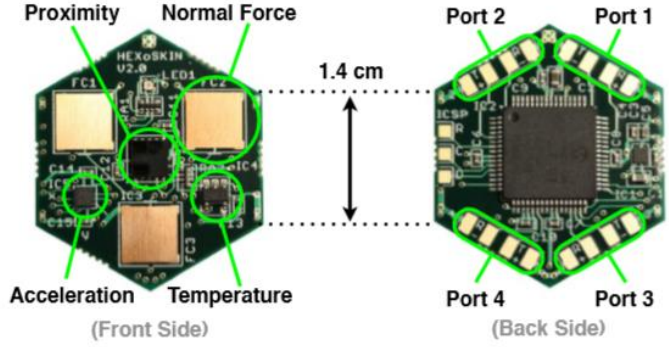

(b)
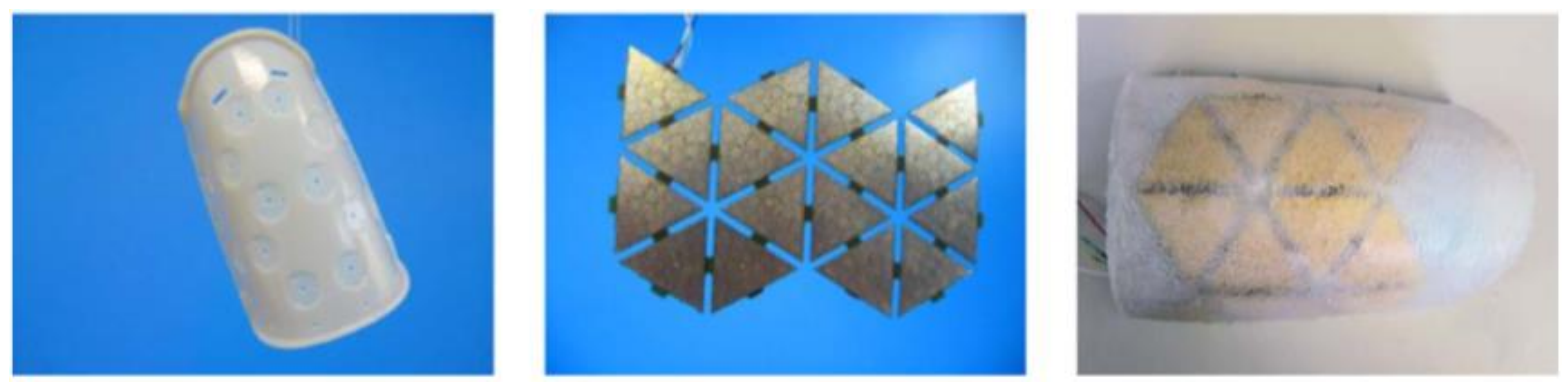

(c)

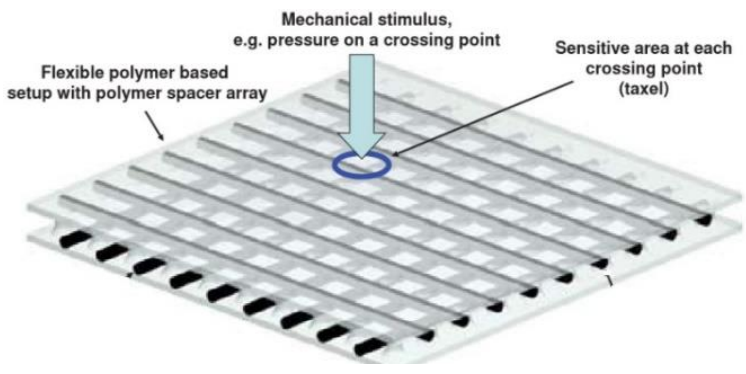

(d)

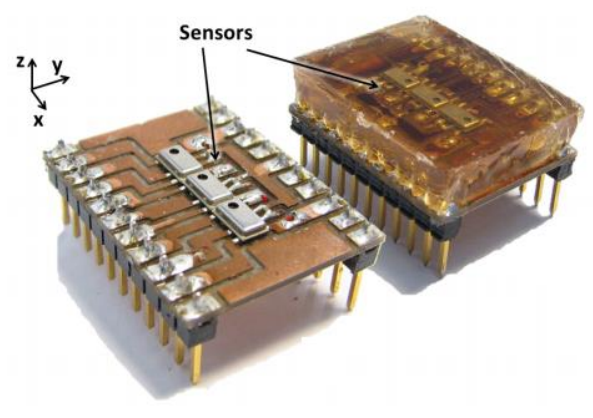

Figure 2-1 Electronic skin examples: (a) HEX-O-SKIN, (b) ROBOSKIN, (c) DLR Artificial

Skin and (d) TakkTile tactile arrays 
DLR artificial skin, developed in Germany, is a stretchable robot skin designed for sensitivity to minute pressures and the ability to withstand high impact collisions [24]. DLR skin utilizes orthogonally layered electrically conductive polymer based circuit tracks (PBCT) for its tactile sensing modality. Compressive deformation on the skin causes the contact area between two cross-linked PCBT to increase resulting in a measureable resistance change at the node. During experiment the DLR skin withstood impact forces up to $50 \mathrm{~N}$ while collecting tactile data at a rate of 409 FPS. The second incarnation of the DLR artificial skin focuses on scalability concerns, specifically related to size, spatial resolution, measuring range and readout frequency [25]. The PCBT are altered to have triangular cross sections to optimize the scalable measuring range and "multiplexers are replaced by analog switches for both the supply and... readout electrodes of the... sensor array". The analog switches enable "dynamic connection of individual row and column electrodes" resulting in "variable receptive fields." This means the resolution of an $n \times n$ array can be set during different tasks and according to the desired readout frequency.

TakkTile are low cost tactile array sensors utilizing barometric pressure sensors on PCB [26]. The PCB sensors incorporates instrumentation amplifiers, analog-to-digital convertors, pressure and temperature sensors and control circuitry encapsulated in a soft polymer [26]. TakkTile sensor arrays are currently available for purchase and can be customized for different array sizes and rubber skin thicknesses.

\subsubsection{Advantages of Soft Robot Skin}

Compliant, or soft, robot skin has many advantages to the hard outer surface of a typical robot. Soft skin better mimics the pliable, almost fluid behavior of human skin and might be better accepted in a social environment with pHRI. Soft skin also can absorb impacts experienced by a robot as it travels through cluttered, unstructured 
environments. Another advantage is the increased sensitivity taxels embedded in soft skin experience. This is highlighted by the phenomena of hyperacuity.

In [27] hyperacuity in robot sensing is researched. Hyperacuity is the ability to detect spatial differences at a distance smaller than the length between adjacent sensors. This ability results from overlapping sensor fields and is naturally present in animals. A given example is braille reading, where users are able to perceive "spatial patterns of a finer resolution than the spacing between touch receptors in the fingertips." This ability was tested on an iCub palm and fingertip implanted with ROBOSKIN tactile sensing modules [22]. Experiments conducted on the iCub demonstrated a 10:1 ratio between position acuity and taxel resolution, e.g., position acuity for the fingertip reached approximately $0.3 \mathrm{~mm}$ while taxel spacing was $4 \mathrm{~mm}$. This demonstrates the advantage of covering tactile sensors with a soft, deformable material in that responses to mechanical input will be spread out across multiple sensors.

Another example of this can be seen in [28] with an optical three-axis tactile sensor. The sensor surface is covered in rubber skin which serves both to protect the sensitive light-emitting diodes (LED) and eliminates insensible areas. Computational finite element method (FEM) was performed on the design and showed that sensitivity to three-axis forces are not decreased.

\subsubsection{Robot Skin Manufacturing Technology}

As much as from improved implementation of current sensing technology, robot skin will also benefit from advances in micro- and nano-scale manufacturing of flexible electronics.

Research in [18] details high-performance, $2.5 \mu \mathrm{m}$ silicone wires and microstructures realized on ultra-thin $(10 \mu \mathrm{m})$ polyimide which can be used for developing integrated circuits (IC) on flexible substrates. The performance of organic and inorganic 
electronics on bendable substrates and plastic foils are discussed in both [29] and [30]. In these studies electronic performance was observed to decrease at a bending radius of $5 \mathrm{~mm}$; resistivity at the same bending radius only changed by $5 \%$ [29]. Piezoelectric flexible tactile sensors printed on ultrathin polyimide were constructed in [31]. The sensors showed a linear response to both sinusoidal stimuli up to 2 Newtons $(\mathrm{N})$ and a $1 \mathrm{~N}$ stimuli from $200-1200 \mathrm{~Hz}$.

\subsubsection{Frubber ${ }^{\circledR}$}

Frubber $\AA^{\circledR}$, a patent of Hanson Robotics Inc., is an artificial skin material used to enhance the realism of social robots. It has been used on multiple robots including Zeno, an interactive robot designed to treat and diagnose children with autism, and an android portrait of Philip K. Dick (PKD), designed for lifelike emotions and expressions during pHRI. Frubber is a porous elastomer with high elongation properties and low tear strength that better mimics the aesthetics of human skin than solid elastomers [32].

\subsection{Research in Viscoelastic Materials}

Any elastic polymer, whether foam or solid, that is used to cover or embed tactile sensors will experience viscoelastic behavior. To accurately model and interpret the signals from these sensors viscoelastic behavior must be understood. In this section we take a look at the different behaviors of viscoelastic material and how they can be modeled.

When a material undergoes small strains, the response is assumed to be elastic. Larger deformations require the consideration of viscoelastic behavior. Viscoelastic materials are materials whose stress-strain relationship depends on time [33]. When deformed, some of the behaviors viscoelastic materials exhibit include creep, relaxation, recovery and other combinations of elastic and inelastic responses (figure 2-2). 
(a)

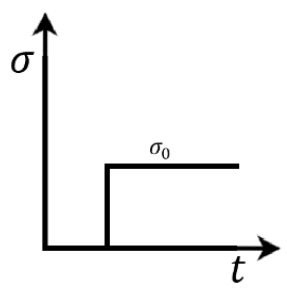

(c)

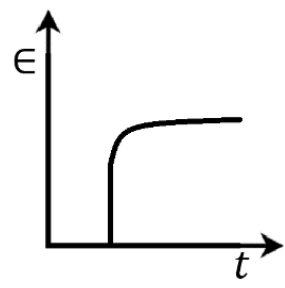

(b)
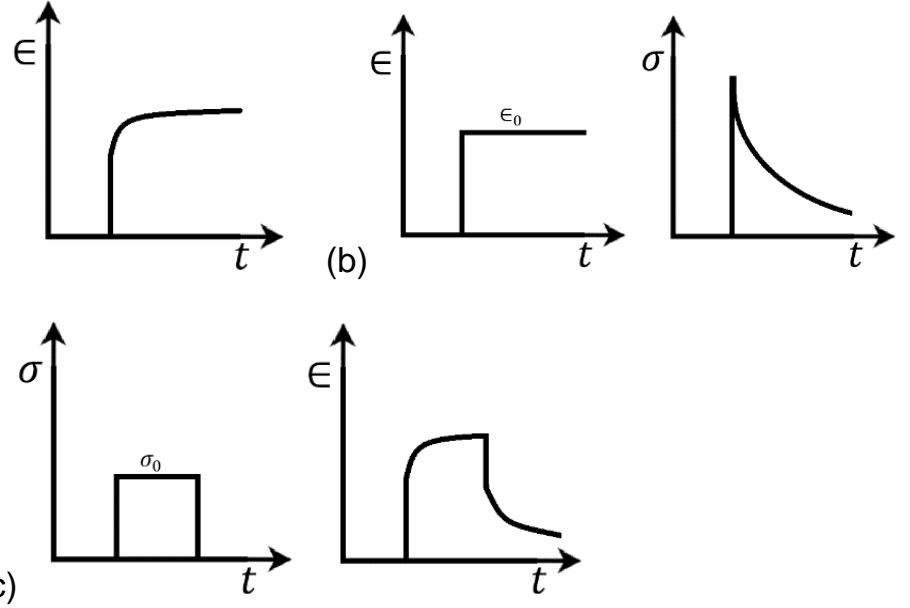

Figure 2-2 Behaviors of viscoelastic material: (a) creep under constant stress, (b) stress relaxation under constant strain, (c) instantaneous and delayed recovery from stress input (figure adapted from [34])

Viscoelasticity has multiple causes. One of the causes in polymers is friction during deformation between entangled and neighboring molecular chains [33]. Under small strains porous elastomers, like Frubber ${ }^{\circledR}$, deform due to the buckling of the cell structure. Straining further causes the expulsion of air from the pores until the cell structures are fully collapsed. During this region the material experiences an increase in density and the stress-strain curve rises significantly.

\subsubsection{Linear Constitutive Models of Viscoelastic Behavior}

Linear constitutive models for viscoelastic materials relate the stress and strain at instant $t$ by linear differential equations with constant coefficients [35]. Elements in the differential model consist of springs and dashpots which represent elastic and viscous elements, respectively. These elements are combined in both parallel and series to model different behavior.

Linear springs follow Hooke's law, 


$$
\sigma=E \in
$$

where $\sigma, E$ and $\epsilon$ represent stress, Young's modulus and strain, respectively.

Linear dashpots follow Newton's law,

$$
\sigma=\eta \frac{d \epsilon}{d t}
$$

where $\eta$ represents Newtonian viscosity.

The Kelvin-Voigt model combines a spring and a dashpot connected in parallel. The elements experience the same strain while the total stress is the sum of the stress from each element. The Maxwell model connects a spring and dashpot in series. Both elements experience the same stress while the total strain is the sum of the individual strains on each element. The Kelvin-Voigt, Maxwell and standard viscoelastic solid rheological models are seen in figure $2-3$. The standard viscoelastic solid model is a Kelvin-Voigt model with the dashpot replaced by a Maxwell model.

(a)

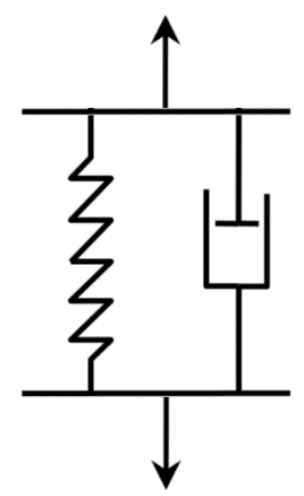

(b)

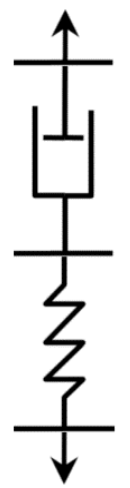

(c)

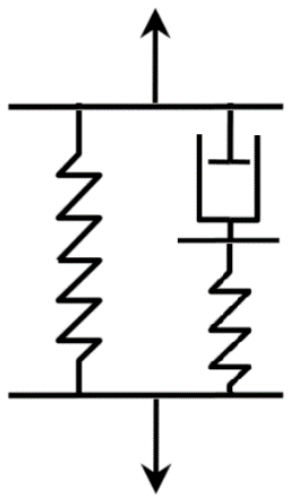

Figure 2-3 Rheological models of (a) Kelvin-Voigt model, (b) Maxwell model and (c) standard viscoelastic solid model

The differential equation describing the stress-strain relationship for the KelvinVoigt model is the following:

$$
\dot{\epsilon}+\frac{E}{\eta} \in=\frac{\sigma}{\eta}
$$


Under a constant stress input $\sigma_{0}$ (applied at $t=0$ ) the strain in a Kelvin-Voigt model experiences creep. After loading the strain slowly approaches a maximum $\frac{\sigma_{0}}{E}$. The equation is:

$$
\in(t)=\frac{\sigma_{0}}{E}\left(1-e^{-\frac{E t}{\eta}}\right)
$$

If the Kelvin-Voigt model is unloaded at $t=t_{1}$, the stress, again, slowly reaches equilibrium and relaxes to its original length. The equation for $t>t_{1}$ is:

$$
\in(t)=\frac{\sigma_{0}}{E} e^{-\frac{E t}{\eta}}\left(e^{-\frac{E t}{\eta}}-1\right)
$$

The stress-strain relationship for a Maxwell model is the following:

$$
\dot{\epsilon}=\frac{\dot{\sigma}}{E}+\frac{\sigma}{\eta}
$$

Under a constant stress input $\sigma_{0}$ (applied at $t=0$ ) the strain in a Maxwell model undergoes an immediate elastic response and a viscous response that increases with time. The equation is:

$$
\in(t)=\frac{\sigma_{0}}{E}+\frac{\sigma_{0}}{\eta} t
$$

The stress for a Maxwell model under constant strain $\epsilon_{0}$ (with an accompanying initial stress $\sigma_{0}$ ) experiences and immediate stress that decays exponentially over time. The equation is:

$$
\sigma(t)=\sigma_{0} e^{-\frac{E t}{\eta}}=E \in_{0} e^{-\frac{E t}{\eta}}
$$

\subsubsection{Hysteresis}

Hysteresis is a nonlinear phenomenon where the relationship between a system's input and output differ between loading and unloading [5]. The relationship between loading and unloading is demonstrated in figure 2-4. 


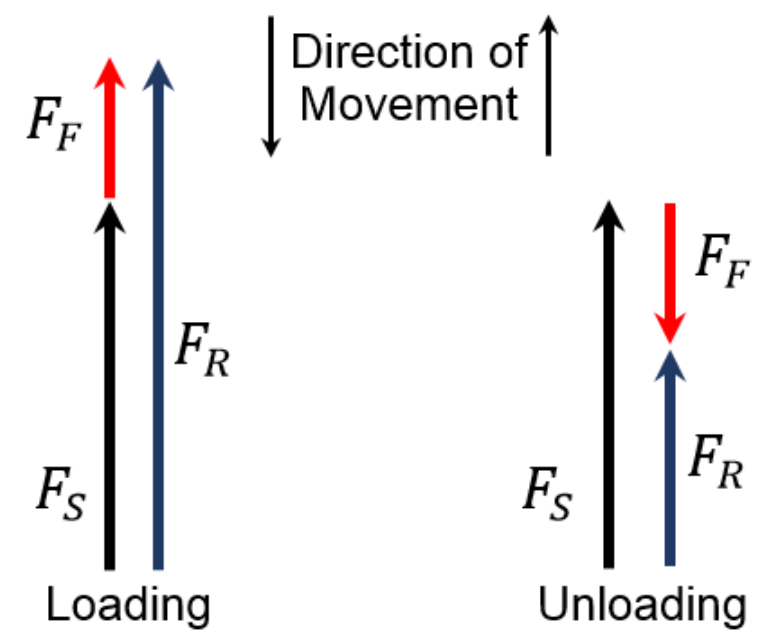

Figure 2-4 The reaction force $F_{R}$ is different during loading and unloading due to the friction force $F_{F}$ opposing the direction of movement. $F_{F}$ is added to the force from the skin $F_{S}$ during compression and subtracted during relaxation

(figure adapted from [36])
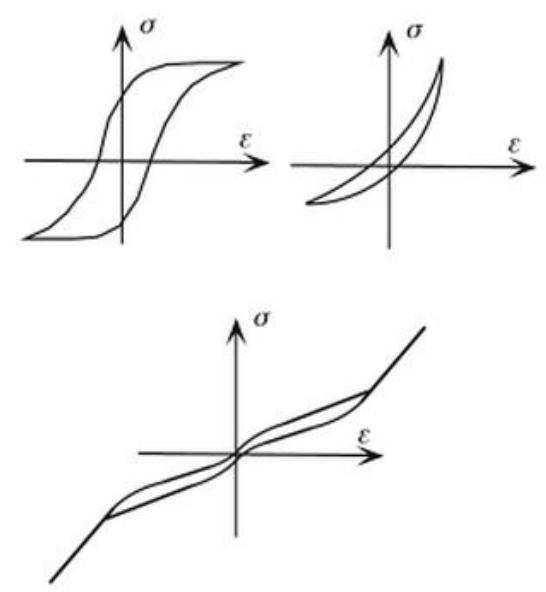

Figure 2-5 Stress $\sigma$ vs. strain $\epsilon$ example graphs of nonlinear viscoelastic materials [33] 
Figure 2.5 showcases the hysteretic behavior of several nonlinear viscoelastic materials. The area inside the loops represents the energy per volume dissipated per cycle [33].

\subsection{Linear Least Squares}

Linear least squares can be used for curve fitting, parameter identification and system model realization [37]. In this thesis, we use a linear least squares algorithm to identify parameters for a reduced-order model composed of a mass element, two springs and two dashpots. The residual error, error between measured values and estimated values, is minimized in a least squares algorithm to estimate the unknown parameters [37]. Below is detailed the process of using linear least squares (taken from [37]).

A set of $m$ discrete measurements is required:

$$
\left\{\tilde{y}_{1}, t_{1} ; \tilde{y}_{2}, t_{2} ; \ldots ; \tilde{y}_{m}, t_{1 m}\right\}
$$

A process $y(t)$ with a proposed mathematical of the form

$$
y(t)=\sum_{i=1}^{n} x_{i} h_{i}(t), \quad m \geq n
$$

with independent basis functions

$$
h_{i}(t) \in\left\{h_{1}(t), h_{2}(t), \ldots, h_{n}(t)\right\}
$$

We represent the measured values $\tilde{y}_{j}$ and the estimated values $\hat{y}_{j}$ with the following equations:

$$
\begin{gathered}
\tilde{y}\left(t_{j}\right)=\sum_{i=1}^{n} x_{i} h_{i}\left(t_{j}\right)+v_{j}, \quad j=1,2, \ldots, m \\
\hat{y}\left(t_{j}\right)=\sum_{i=1}^{n} \hat{x}_{i} h_{i}\left(t_{j}\right), \quad j=1,2, \ldots, m
\end{gathered}
$$

where $v_{j}$ and $\hat{x}_{i}$ represent the measurement error and estimated states, respectively [37]. 
The measured values $\tilde{y}_{j}$ and the estimated values $\hat{y}_{j}$ can be related through the following equation:

$$
\tilde{y}\left(t_{j}\right)=\sum_{i=1}^{n} \hat{x}_{i} h_{i}\left(t_{j}\right)+e_{j}, \quad j=1,2, \ldots, m
$$

defining $e_{j}$ as the residual error

$$
e_{j} \equiv \tilde{y}_{j}-\hat{y}_{j}
$$

The compact matrix form of the previous equation for $\tilde{y}_{j}$ can be expressed as

$$
\widetilde{\boldsymbol{y}}=\boldsymbol{H} \widehat{x}+\boldsymbol{e}
$$

with the arrays equal to:

$$
\begin{gathered}
\tilde{\boldsymbol{y}}=\left[\begin{array}{llll}
\tilde{y}_{1} & \tilde{y}_{2} & \ldots & \tilde{y}_{m}
\end{array}\right]^{T} \\
\boldsymbol{e}=\left[\begin{array}{llll}
e_{1} & e_{2} & \ldots & e_{m}
\end{array}\right]^{T} \\
\widehat{\boldsymbol{x}}=\left[\begin{array}{llll}
\hat{x}_{1} & \hat{x}_{2} & \ldots & \hat{x}_{m}
\end{array}\right]^{T} \\
\boldsymbol{H}=\left[\begin{array}{cccc}
h_{1}\left(t_{1}\right) & h_{2}\left(t_{1}\right) & \ldots & h_{n}\left(t_{1}\right) \\
h_{1}\left(t_{2}\right) & h_{2}\left(t_{2}\right) & \ldots & h_{n}\left(t_{2}\right) \\
\vdots & \vdots & & \vdots \\
h_{1}\left(t_{m}\right) & h_{2}\left(t_{m}\right) & \ldots & h_{n}\left(t_{m}\right)
\end{array}\right]
\end{gathered}
$$

The optimum choice for the unknown parameters is the one that minimizes the sum square of the residual errors $\mathrm{J}$ :

$$
J=\frac{1}{2} \boldsymbol{e}^{T} \boldsymbol{e}
$$

$J$ can be reworked into the following form (with substitution of the compact matrix form for $\tilde{y}_{j}$ ):

$$
J=J(\widehat{\boldsymbol{x}})=\frac{1}{2}\left(\widetilde{\boldsymbol{y}}^{T} \widetilde{\boldsymbol{y}}-2 \widetilde{\boldsymbol{y}}^{T} \boldsymbol{H} \widehat{\boldsymbol{x}}+\widehat{\boldsymbol{x}}^{T} \boldsymbol{H}^{T} \boldsymbol{H} \widehat{\boldsymbol{x}}\right)
$$

Using matrix calculus differentiation and applying the rules for global minimum of the quadratic function $J$ present the following requirements for the Jacobian of $J$ :

$$
\nabla_{\widehat{x}} J \equiv \boldsymbol{H}^{T} \boldsymbol{H} \widehat{\boldsymbol{x}}-\boldsymbol{H}^{T} \widetilde{\boldsymbol{y}}=\mathbf{0}
$$


With this form we can solve for $\widehat{x}$ to obtain the equation for the optimal parameters to minimize the residual error:

$$
\widehat{\boldsymbol{x}}=\left(\boldsymbol{H}^{T} \boldsymbol{H}\right)^{-1} \boldsymbol{H}^{T} \widetilde{\boldsymbol{y}}
$$


Chapter 3

\section{Description of Experimental Testbed}

To gather measurements for many experiments in a repeatable, efficient manner we selected a platform that has been developed with extensively and had many options for hardware capabilities. The platform of our experimental testbed is a National Instruments (NI) compactRIO (cRIO) with accompanying programming package LabVIEW (LV). In our testbed the cRIO gathers real-time measurements from multiple sensors and controls a Newport actuator. The completed testbed and system architecture can be seen in figure 3-1 and 3-2. Two types of sensors are used for our experiments: FlexiForce force sensors and a Transducer Techniques Inc. (TTI) load cell. The FlexiForce sensor acts as an embedded taxel in robot skin and measures what load the robot perceives. The force applied to this sensing element is measured by the load cell. In this section we will detail each component of the experimental testbed including the programs that were used to run the experiments.

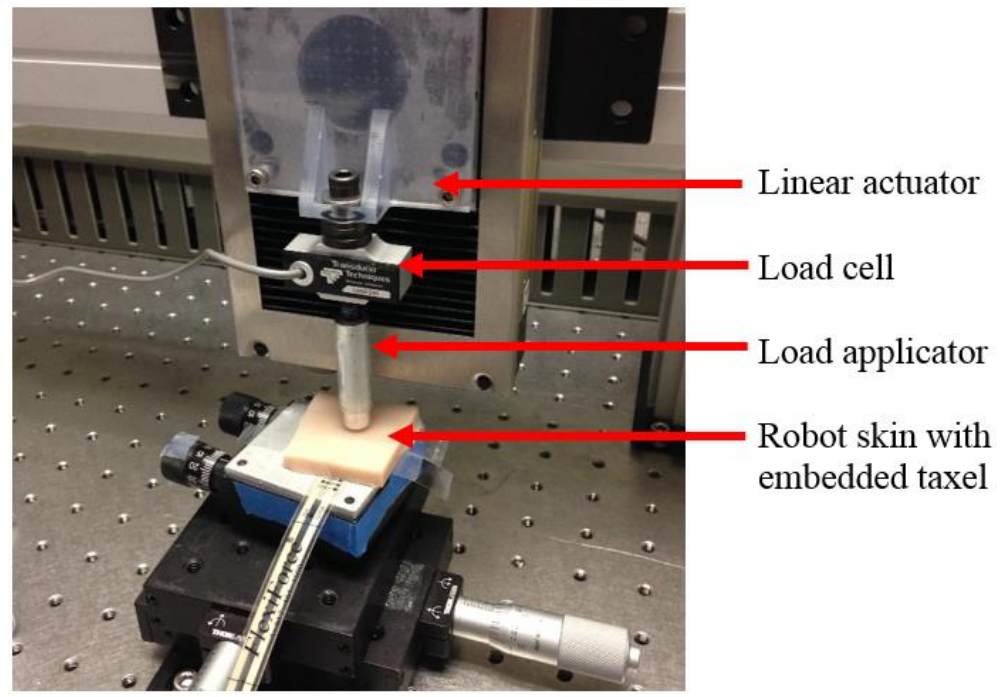

Figure 3-1 Experimental testbed: the load cell and load applicator are mounted to the linear actuator to depress into the robot skin 


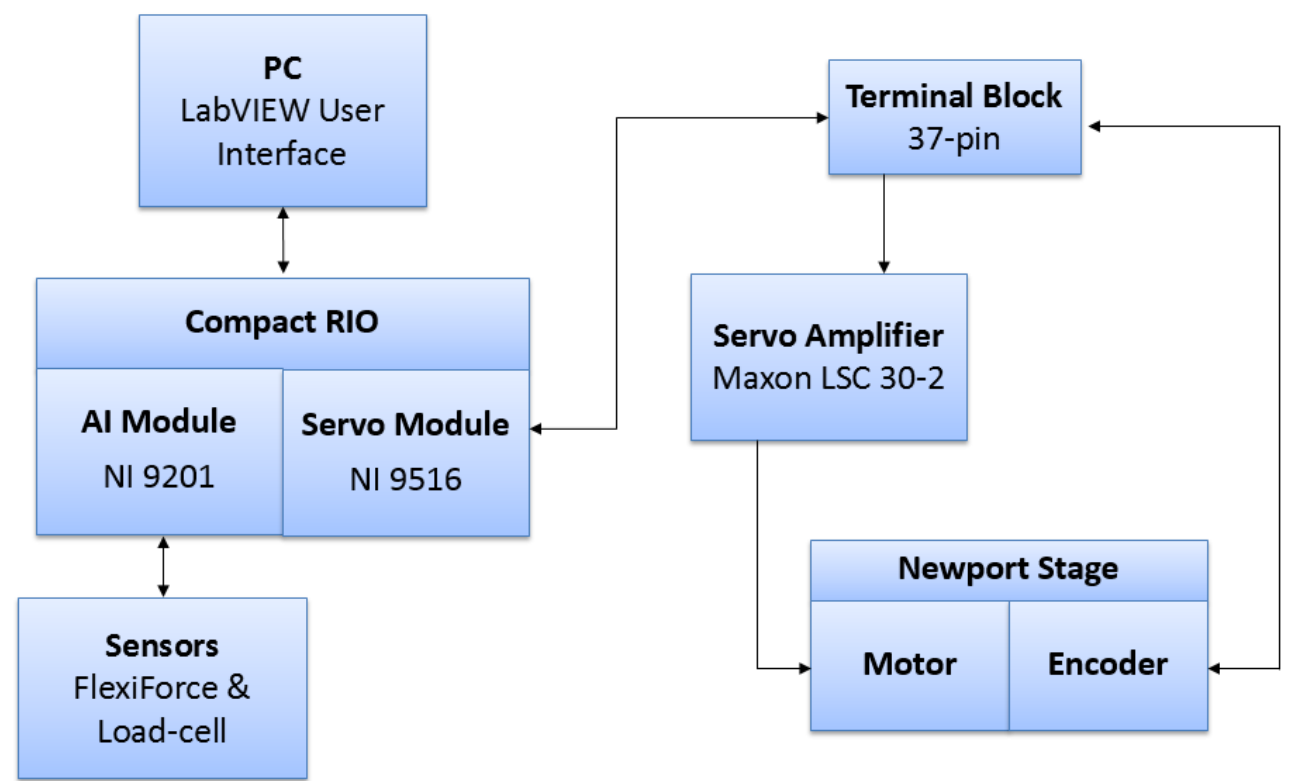

Figure 3-2 Testbed system architecture

\subsection{Testbed Controller}

The following section details the hardware used for controlling the experiments we conducted in this thesis.

To gather measurements from multiple sensors and control an actuator in realtime, a NI cRIO was selected. The model number of the controller is NI cRIO-9074 and can be seen in figure 3-3.

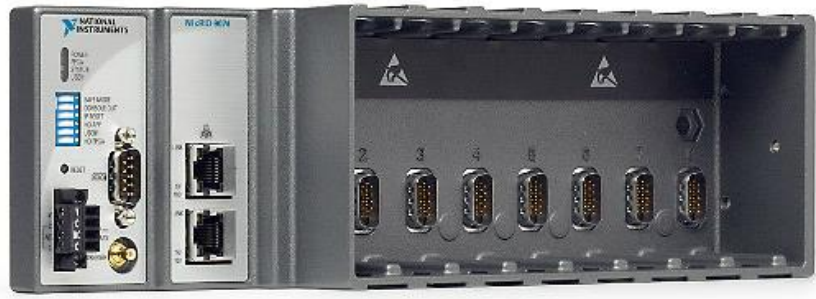

Figure 3-3 National Instruments cRIO-9074 [38]

The $\mathrm{cRIO}$ is a reconfigurable, deterministic data acquisition device that can be programmed with NI LV graphical programming. It has a $400 \mathrm{MHz}$ real-time processor 
and an 8-slot FPGA chassis housing hot-swappable modules [38].The advantage of this controller is in its ability to gather synchronous time-stamped measurements from multiple sensors while also controlling actuators or other devices in a testbed. Synchronous, time-stamped measurements are essential to the model fitting that will be discussed in Chapter 4. Two NI C Series modules were used in our testbed: the NI 9201 Analog Input Module and the NI 9516 Servo Drive Interface Module (Figure 3-4).

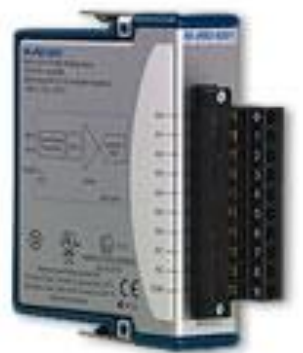

(a)

Figure 3-4 (a) NI 9201 Al Module [39], (b) NI 9516 Servo Drive Interface [40]

The NI 9201 is an 8-channel, 12-bit analog to digital converter with a range of \pm $10 \mathrm{~V}$. This module is used for recording the measurements from the two force sensors in the testbed. The module has a maximum sampling rate of $500 \mathrm{kS} / \mathrm{s}$, or $62.5 \mathrm{kS} / \mathrm{s}$ per channel and a resolution of $4.88 \mathrm{mV}$ [39].

The NI 9516 is a servo drive interface used for sending signals to and receiving signals from the linear actuator and servo amplifier. It has dual encoder feedback with a $50 \mu$ s control loop rate along with spline interpolation, position and torque control [40]. The NI 9516 is used in conjunction with a Maxon servo amplifier, model number LSC 30/2 (Figure 3.5). The Maxon servo amplifier receives drive signals from the NI 9516 and sends the corresponding current to the actuator. With feedback from the actuator's encoder the servo amplifier can adjust the transmitted current to reduce the position error of the actuator. 


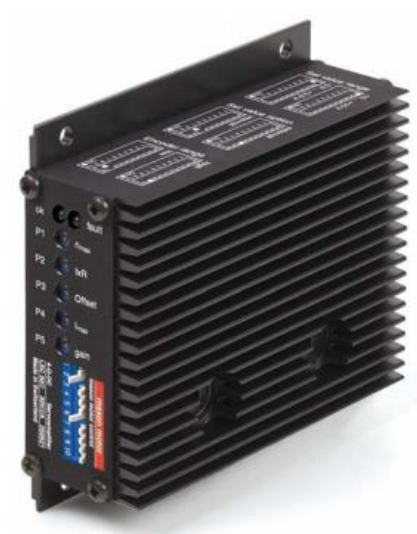

Figure 3-5 Maxon LSC 30/2 servo amplifier [41]

The linear actuator is a Newport UTMCC1HL. The actuator has a $150 \mathrm{~mm}$ range and on-axis accuracy of $5 \mu \mathrm{m}$. The maximum travel speed is $20 \mathrm{~mm} / \mathrm{sec}$. The actuator has a single 2000pts/rev encoder with a resolution of $1 \mu \mathrm{m}$.

\subsection{LabVIEW Motion Control and Measurement Collection}

\subsubsection{Motion Control}

Motion control "is a complex system with several nested control loops" consisting of motion control software, communication module, servo amplifier, motor with transmission device and feedback devices [6]. An architectural overview of motion control software and hardware is seen in figure 3.6.

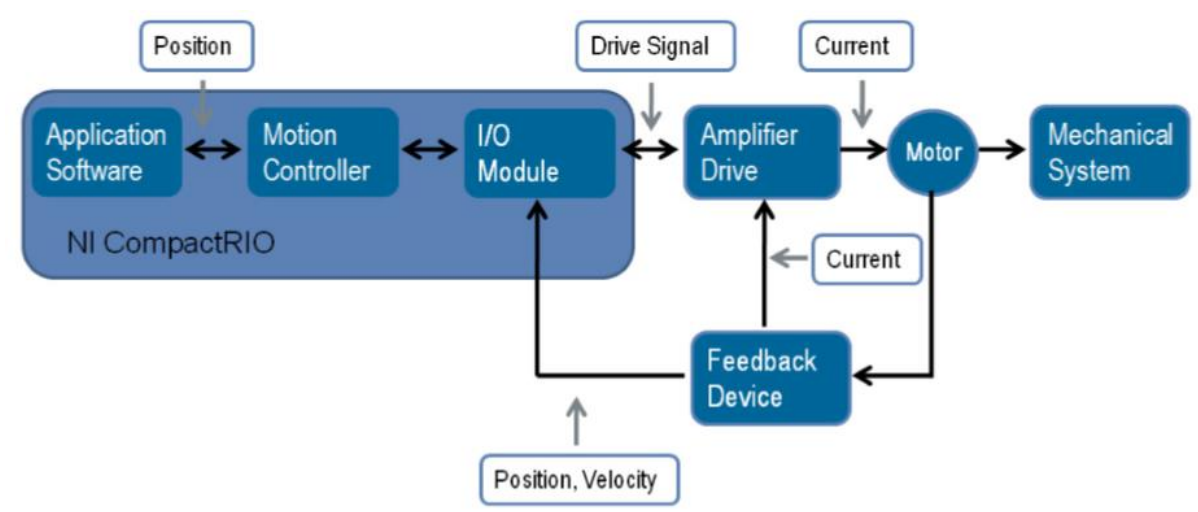

Figure 3-6 Motion control for cRIO 
The motion control software algorithms transmit desired motion profiles from the user into motion control commands for the communication $1 / O$ module [6]. For our experiment the motion control software running on the cRIO was NI SoftMotion. SoftMotion is a high-level application programming interface (API) that implements supervisory control and trajectory generation [6]. The top level supervisory control loop includes initialization of the system, event handling, and fault detection [6]. The deterministic nested trajectory generation loop receives commands from the supervisory control loop and creates location set points for a path profile based on user input [6].

The communication module we used was the NI 9516 servo drive interface. The NI 9516 includes a processor to perform spline interpolation from set points sent by the trajectory generation loop. The servo drive sends drive command signals to the servo amplifier and receives encoder feedback for position control. The servo amplifier for our testbed is the Maxon LSC 30/2 which converts drive commands from the NI 9516 to highcurrent motor signals to control the linear actuator.

\subsubsection{Motion Control Programs}

LV programs, or Virtual Instruments (VI), were created to provide user input for motion generation of the motion control system and for measurement collection. Several VIs were created to perform the following movements: single step input; stair-step input; user set ramp profiles for random input; sinusoidal motion. In addition, a VI was created to implement incremental force control.

During each experiment, the VI allowed a user to view the current position of the linear actuator, force reading from the load cell and force reading from the taxel. An example of the front panel on a motion control $\mathrm{VI}$ is seen in figure 3-7. This $\mathrm{VI}$ implements straight-line movement with a user able to set the position increment, velocity and acceleration. Because the linear actuator does not include a tachometer, velocity and 
acceleration are calculated through the finite difference method of position. The front panel includes an execute button for initializing and starting movement and a stop button for halting the progression of the current move. The front panel tracks the number of both the measurement acquisition loop cycle and the UI update cycle. The measurement acquisition loop number represents the number of iterations the timed-loop recording measurements from the encoder and force sensors has undergone; the UI update loop number represents how many times the UI has updated. The UI loop intentionally runs slower than the acquisition loop and is given lower priority on the cRIO real-time controller. A file path input allows the array of collected measurements to be named for each experiment. The files are stored on the cRIO and must be saved under "C:Ifile_name". Once stored the files are downloaded from the cRIO through NI Measurement and Automation Explorer (MAX).

All the processes in the VI run inside a timed-loop, synchronized to the scan engine of the cRIO. The scan engine is used during LV projects utilizing Scan Mode which is optimal for control loops lower than $500 \mathrm{~Hz}$ and accessing single-point I/O data. During an experiment multiple timed loops can be run at the same time. Loops acquiring and storing measurements from one of the devices and loops for motion commands were set to the same loop time as the scan engine, $100 \mathrm{~Hz}$. Loops updating the user interface iterated every 2 periods of the scan engine, or at $50 \mathrm{~Hz}$. The acquisition and recording of device measurements was the most important task for each VI. This is conveyed to the cRIO by setting priority levels for timed-loops. The acquisition timed-loop has the highest priority level at 100 , followed by the motion command loops at 99 , then the UI loop at 50 . A level of 100 priority ensures that the cRIO always records measurements from the encoder and force sensors; only when that task is completed will the cRIO continue on to the motion command signals and then update the UI. These priority levels were set for 
user assurance, and are not a major issue considering the slow loop rate of the $\mathrm{VI}$ at $100 \mathrm{~Hz}$

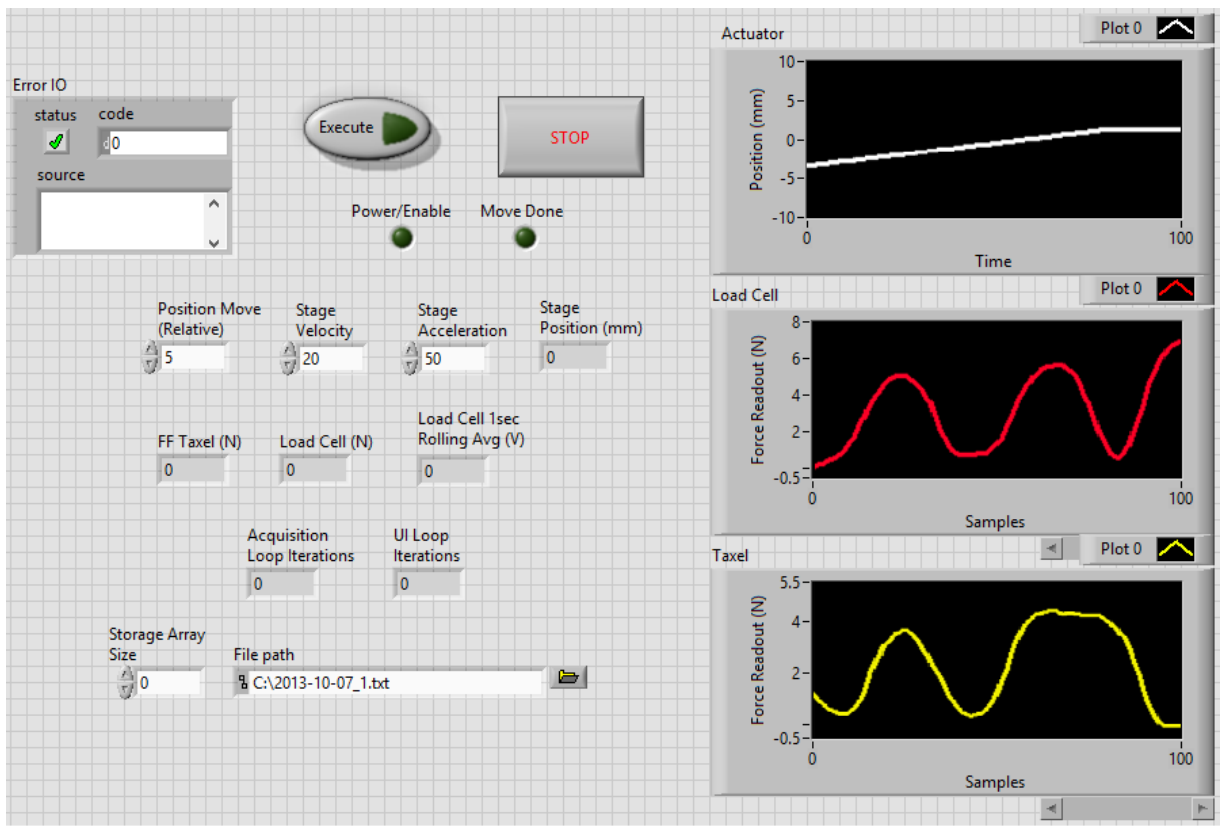

Figure 3-7 Front panel of motion control VI

Each VI used for experiments contained a loop for acquiring and storing measurements from the encoder and force sensors. This loop was synchronized to the scan engine and given highest priority. Readings for the force sensors are gathered from the analog input module and assigned as global variables. The stage position variable is the position of the bottom surface of the plunger and is read from an encoder function block. Global variables can be accessed from any loop in the VI. The size of the storage array is set from the front panel and is set based off experience. To determine the time that has elapsed between consecutive loops, the Execution Time loop was created; this can be seen on the far right side of figure 3-8. Values are stored in the storage array based upon the iteration number of the timed-loop, which corresponds to a specific row. 
Once the Stop button has been pressed on the front panel the array is compiled into a tab-delimited file and sent to the specified file path.

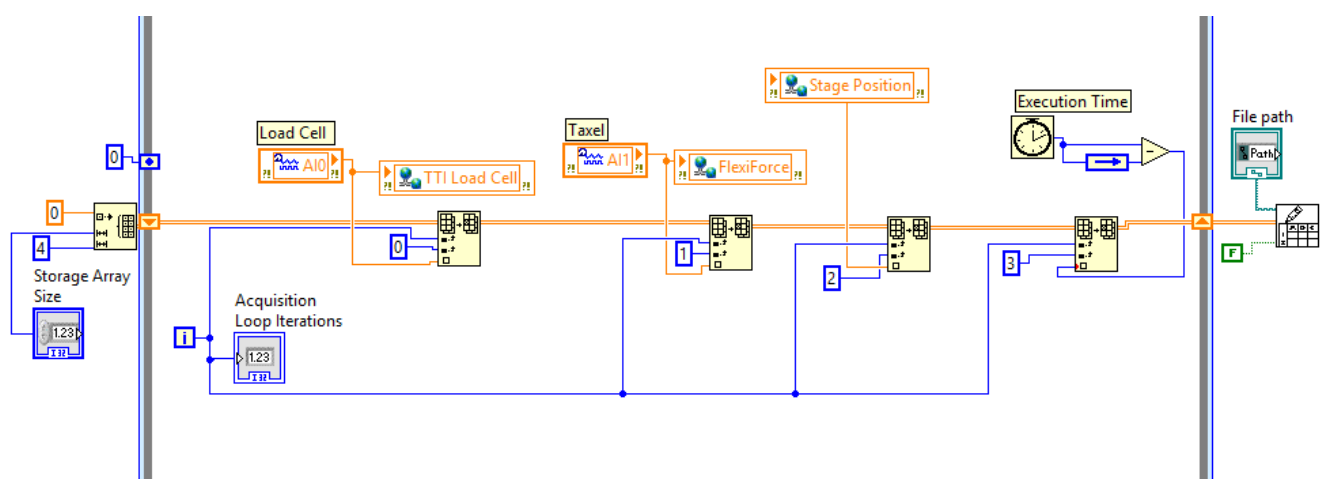

Figure 3-8 Timed-loop for measurement collection \& storage

Both single step input and stair-step input movements are achieved through the use of the Straight-Line Move (figure 3-9) function block (FB). First, after the Execute button has been pressed on the UI, all motion is initiated with the Power FB. The Power FB enables the servo drive and movement along the specified axis. After initialization a signal is sent to the Straight-Line Move FB executing a straight-line move to the relative or absolute position set by the user. The user can also specify the velocity and acceleration for each move in the UI.

Figure 3-10 shows a FB structure similar to the straight-line move, but designed to perform repeated straight-line moves. Specifically, this program performs a stair-step pyramid type movement which was used for calibrating the taxels. The load applicator descends a certain number of times into the taxel (holding a user-specified length of time) and then reverses course. A case structure was required to implement consecutive movements with a hold-time in-between. 


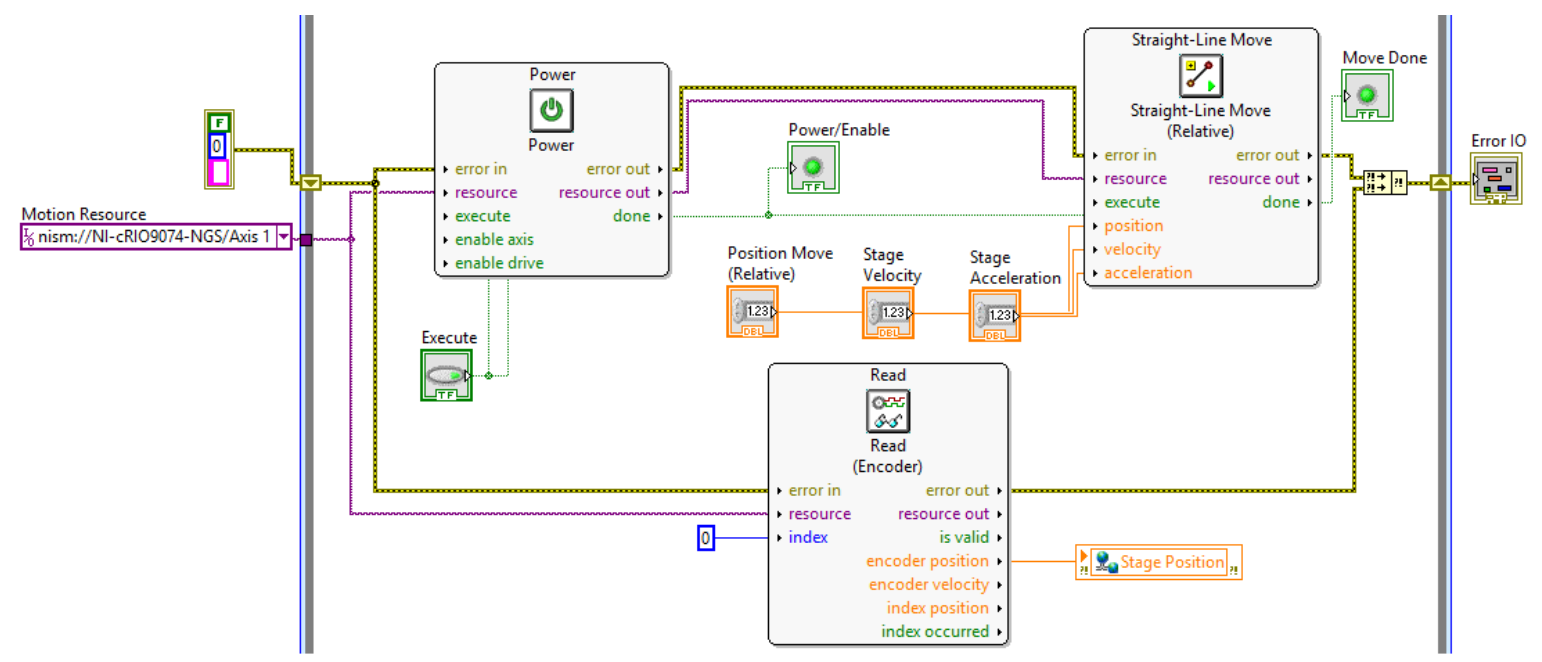

Figure 3-9 Function block structure for straight-line move

(a)

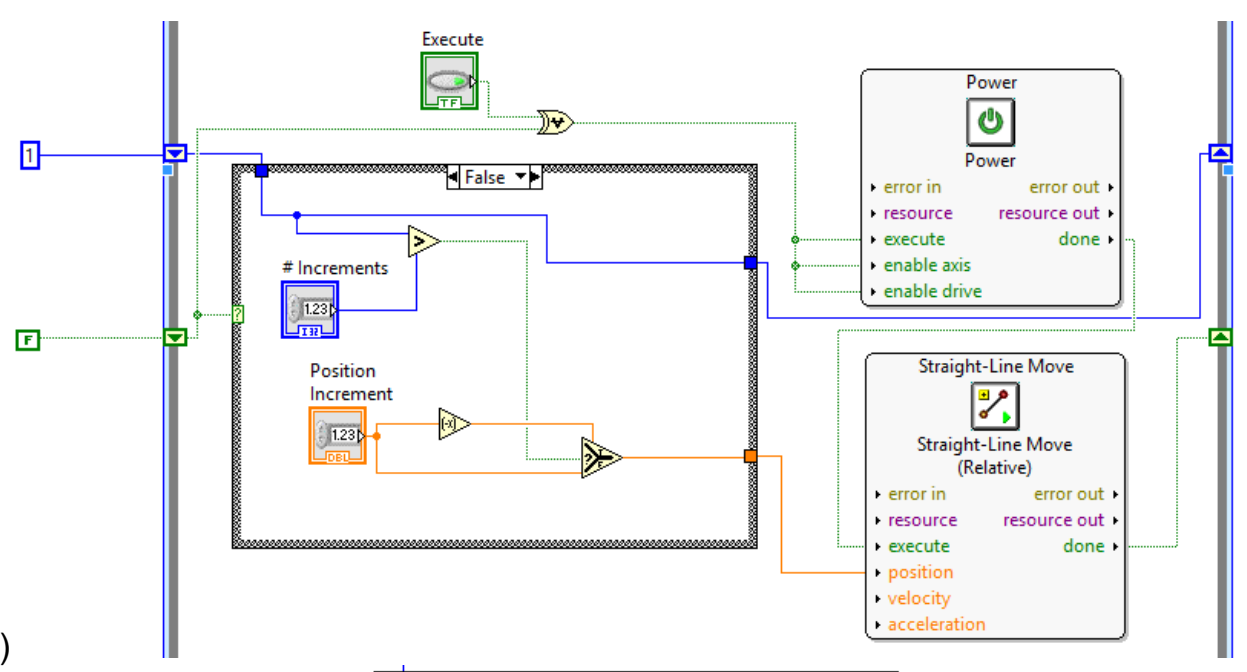

(b)

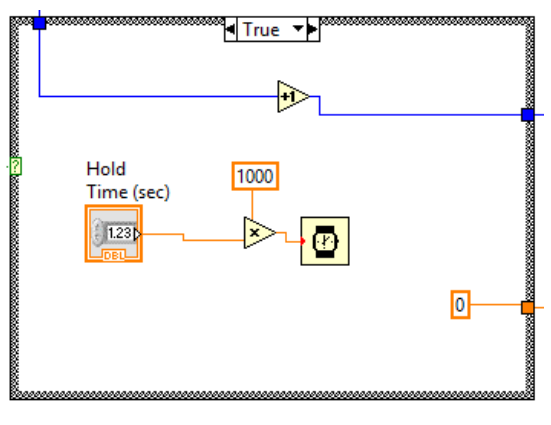

Figure 3-10 Function block structure for straight-line move: (a) case structure false, (b) case structure true 
Sinusoidal motion is completed in a similar manner to straight-line movements but instead utilizes the Arc Move FB (figure 3-11). The arc move is designed to be implemented with two perpendicular stages. However, when used with just one stage it can perform sinusoidal motion. A program implementing the Arc Move FB was used in parameter identification for the reduced-order models.

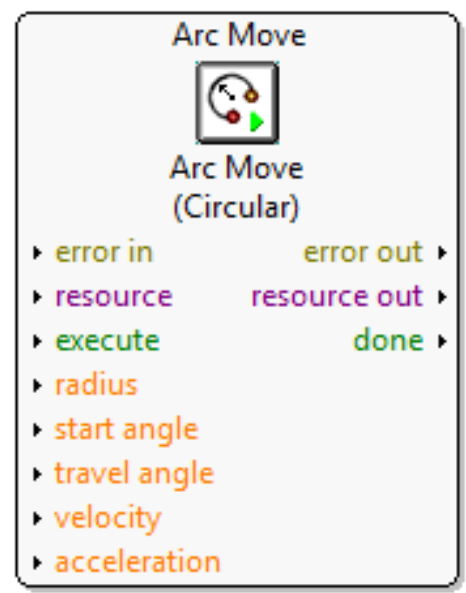

Figure 3-11 Arc Move function block for sinusoidal motion input

Another program was created utilizing the Straight-Line Move FB that was used for applying repeated step inputs. The user set the position increment for the load applicator along with the stage velocity and acceleration; the user also set the length of time the load applicator should depress into the sample and/or taxel. This repeated stepinput program works similar to the straight-line move program except the position increment is fed to the Straight-Line Move FB with the case structure shown in figure 312. The position increment fed to the Straight-Line Move FB alternates between a negative and positive value of the position increment and zero.

A program was written to implement pseudo-random motion input so we could validate the reduced-order models. The Straight-Line Move FB is utilized as in previous cases but the case structure providing user-set position moves has changed (figure 3- 
13). The pseudo-random position moves are determined before the experiment and then typed into an array on the UI. The program then grabs the increments from the array and sends the appropriate move to the Straight-Line Move FB.
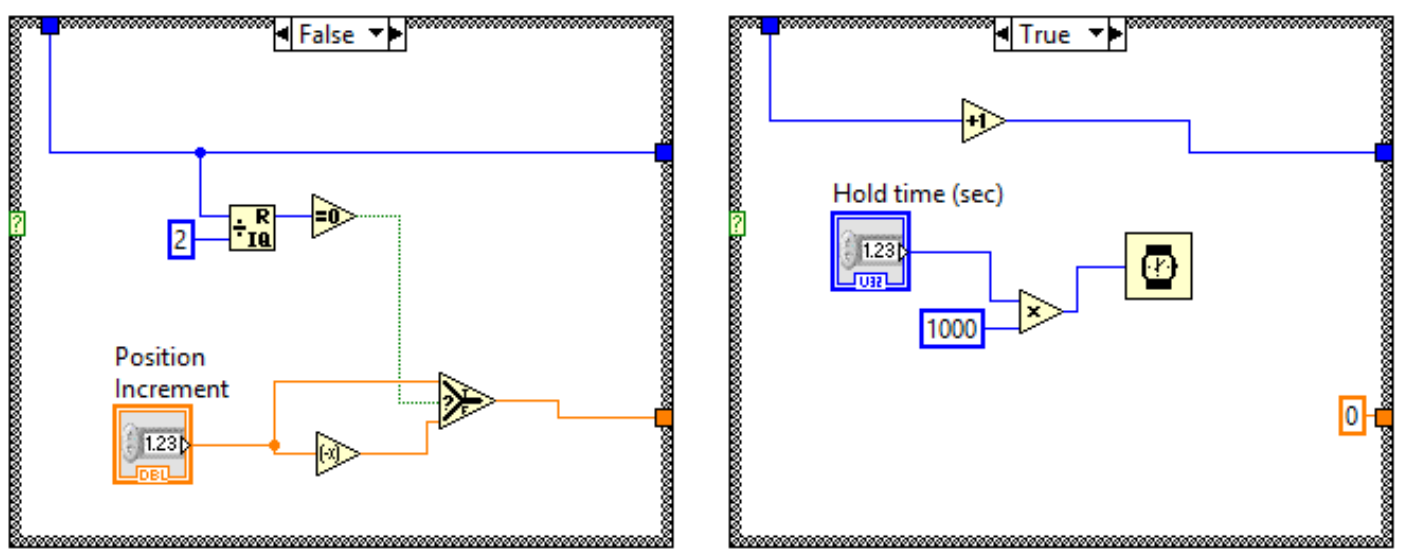

Figure 3-12 Repeated step-input case structure
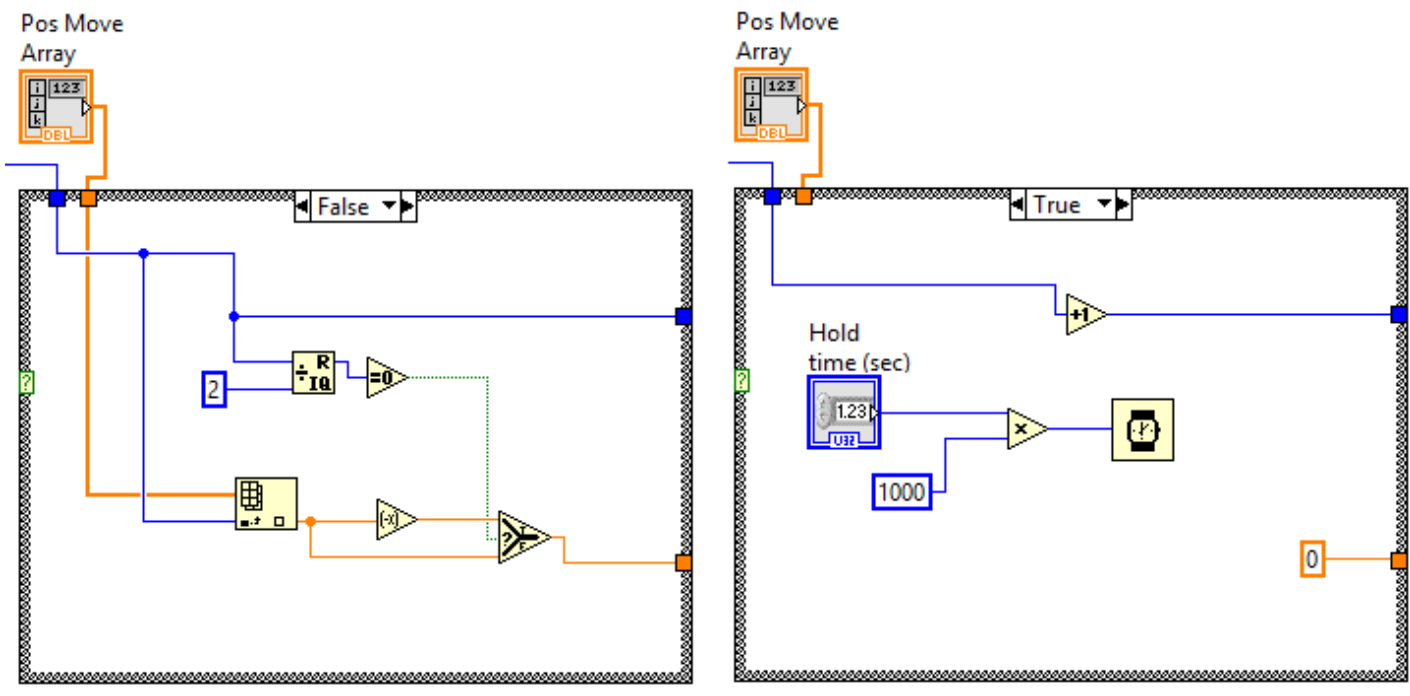

Figure 3-13 Pseudo-random motion input case structure

The previous VIs implemented position control of the plunger using encoder feedback. If instead a user wanted to control the specific force applied to a skin sample or taxel another method must be used. An incremental force controller was developed for 
the testbed to provide position-based force control. An incremental force controller uses the difference between a desired and measured force to provide a user-set position increment to the motion controller. Advantages of the incremental force controller are that it is easy to implement, robust and effective even if a system is nonlinear; disadvantages include the slow response of the system and chatter as a function of the position increment [42]. For implementation in the VI the desired force, set by the user, is compared to the load cell force global variable measurement (figure 3-14). The sign of the error determines the direction of the user-set position increment sent to the StraightLine Move FB.

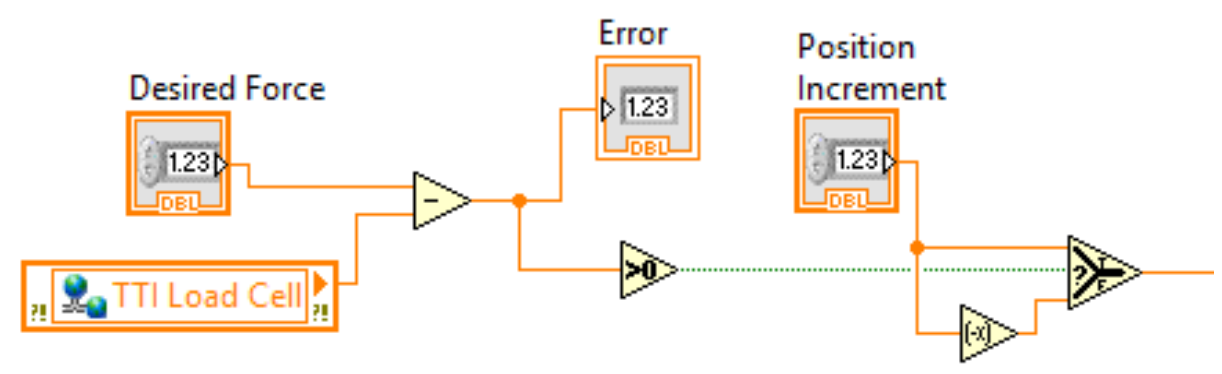

Figure 3-14 Incremental force controller

While the linear actuator has a home limit switch it was not utilized in our testbed. Instead, to set the zero position at the top surface of skin samples and taxels a rolling average loop was implemented. The rolling average loop records the previous 100 measurements from the load cell and averages them. The implementation is shown in figure 3-15. The zero position of skin samples and taxels was always set to the point where the load cell had a rolling average of $0.01 \mathrm{~V}$. When the load cell reading reached this number, the encoder position was set to zero on the axis configuration panel. 


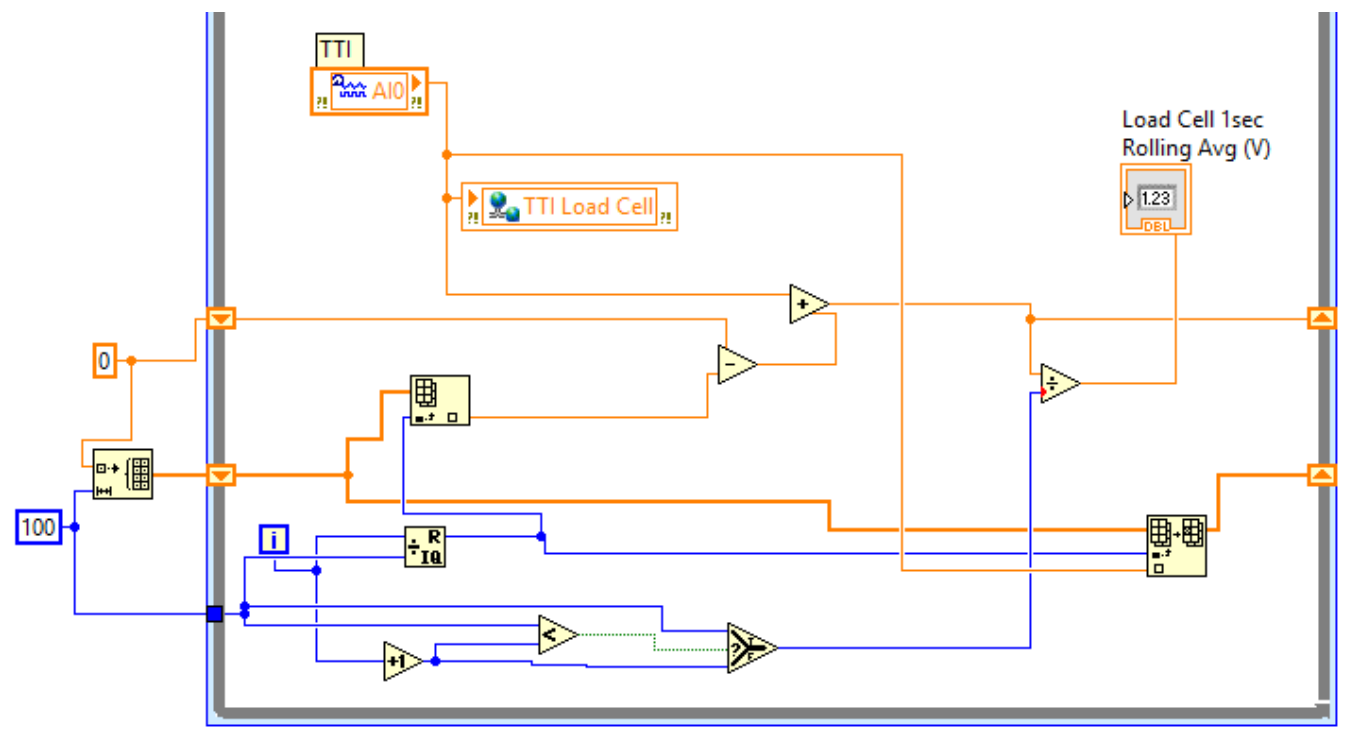

Figure 3-15 Loop for calculating the average of the previous 100 measurements 3.3 Force Sensors

For our experiments two different sensors were used: a Tekscan FlexiForce A201 force sensor representing a robot taxel and a TTI MLP load cell to measure the applied force to the taxel.

\subsubsection{FlexiForce Force Sensor}

The Tekscan FlexiForce A201 force sensor is a piezoresistive, flexible, thin printed circuit (figure 3-16). Piezoresistive materials respond to strain with a change in resistance across the material, a useful property for small sensing elements. As the strain is increased on a piezoresistive material, corresponding to an increase in applied force, the resistance decreases. Resistance is inversely proportional to conductance which is what is used to measure the change in force on the taxels. As the force increases on the sensing element of the taxel the conductance linearly increases. This change is measured and converted to a voltage reading through a drive circuit.

The taxels have the following specifications [43]: 
- Linearity error: $< \pm 3 \%$

- Repeatability: $< \pm 2.5 \%$ of full scale

- Hysteresis: $< \pm 4.5 \%$ of full scale

- Drift: $<5 \%$ per logarithmic time scale

- Response time: $<5 \mu \mathrm{s}$

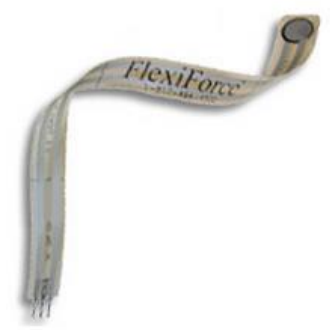

Figure 3-16 Tekscan FlexiForce A201 [43]

The taxels have a sensing area $9.53 \mathrm{~mm}$ in diameter and a thickness of $0.203 \mathrm{~mm}$. The taxels used in our experiments have a 25lbs capacity. Due to the high part-to-part variability of the FlexiForce sensors, as high as $40 \%$, calibration of each sensor is a necessity [44]. Calibration of the taxels is discussed in section 4.1.

To convert the change in resistance of the taxel to a measureable change in voltage, a Phidgets 1120 FlexiForce Adapter was used (figure 3-17) [45]. The Phidgets 1120 , powered by a $5 \mathrm{~V}$ source, converts the analog signal of the taxel resistance change to an analog voltage of $0-5 \mathrm{~V}$. This $0-5 \mathrm{~V}$ output signal is wired into a terminal on the $\mathrm{NI}$ 9201. As previously mentioned, the resolution of the NI 9201 is $4.88 \mathrm{mV}$. With the $0-5 \mathrm{~V}$ output of the Phidgets corresponding with the 0-25lbs capacity of the taxel, the sensing resolution of the taxel in our system is approximately $0.107 \mathrm{~N}$. 


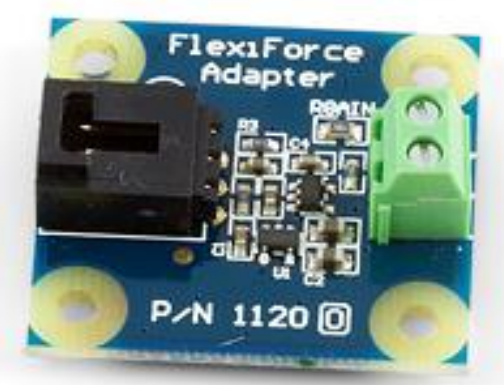

Figure 3-17 Phidgets 1120 FlexiForce Adapter

\subsubsection{Taxel Calibration}

The FlexiForce force sensors were used to represent embedded taxels in robot skin. Because the relationship between the taxel signal conditioner output and the applied force on the taxel is unknown, each taxel must first be calibrated before experiment. Each taxel had an incrementally increasing force applied to its surface resulting from the plunger pushing into the taxel. An example of the deformation experienced by the taxel, in compression, is shown in figure 3-18. The sensors are compressed up to $87 \%$ of their original thickness of $0.203 \mathrm{~mm}$.

The reaction force applied to the plunger is recorded by the load cell which is pre-calibrated. The load cell voltage output is related to applied force through the following equation:

$$
F_{L C}=V_{L C} \times \frac{25 l b f}{8 V} \times \frac{4.44822 N}{1 l b f}
$$

As seen in figure 3-19, the relationship between taxel voltage output and applied force is nearly linear and a linear relationship was used for analysis. To determine the slope between the two the load cell response is plotted against the voltage output of the taxel. Four calibration runs were performed on four different taxels. The equation relating taxel voltage output to the applied force is: 


$$
F_{\text {Taxel }}=V_{\text {Taxel }} \times \text { Slope }
$$

Table 3-1 shows the calibration results of four separate taxels. Taxel 1 and taxel 3 calibration tests were performed all in the same day while taxel 2 and taxel 4 tests spanned multiple days. The calibration results show high sensor-to-sensor variation and low variation between tests on a specific taxel. The maximum standard deviation for any of the taxel calibrations was $0.620 \mathrm{~N} / \mathrm{V}$; the minimum was $0.0266 \mathrm{~N} / \mathrm{V}$. This analysis shows that while taxels can experience up to $40 \%$ sensor-to-sensor variation, they are individually consistent and a slope calculated through calibration experiments can be used for analysis if we take into account which taxel was used.

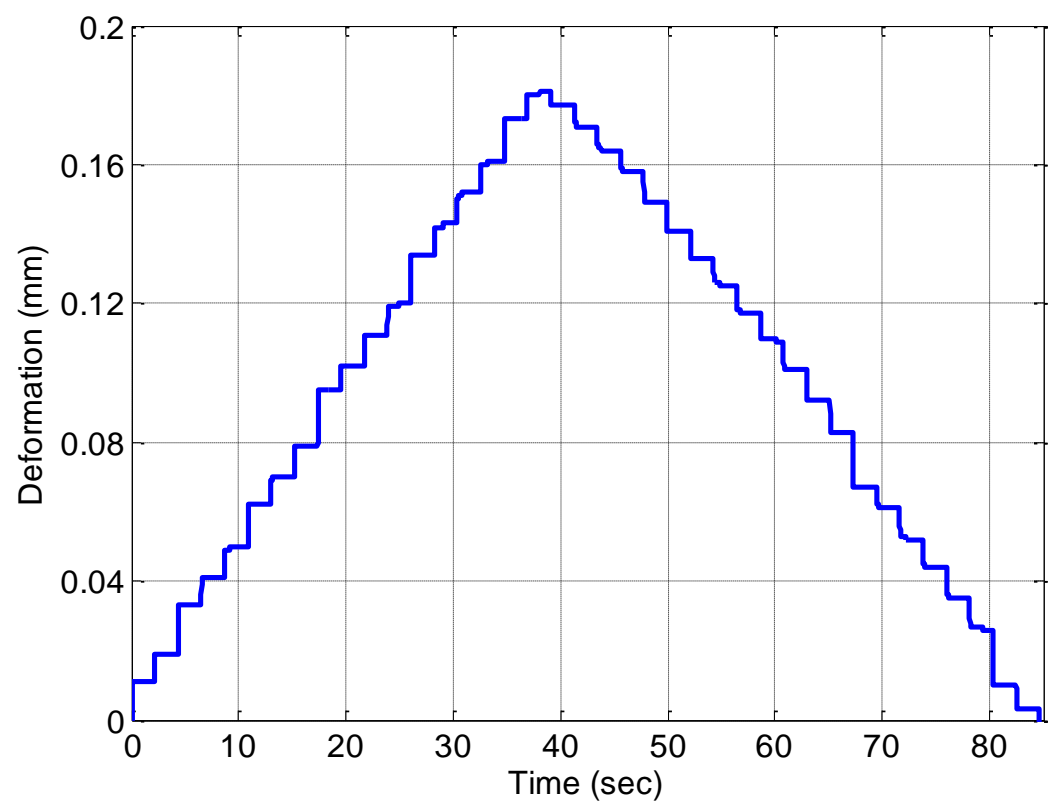

Figure 3-18 Example of the deformation of a single taxel during calibration

The response of each taxel undergoes hysteresis because of the deformable material present inside the sensor (figure 3-20). However, the effect is small enough (maximum 6\% difference between upper and lower curve) that it is ignored for our analysis. 
Table 3-1 Slope values calculated from calibration curves of taxel voltage output vs. applied force

\begin{tabular}{|c|c|c|c|c|}
\hline Units: $N / V$ & Taxel 1 & Taxel 2 & Taxel 3 & Taxel 4 \\
\hline Experiment 1 & 9.413 & 14.08 & 10.52 & 14.04 \\
\hline Experiment 2 & 9.443 & 14.26 & 9.975 & 14.20 \\
\hline Experiment 3 & 9.368 & 13.36 & 10.29 & 12.75 \\
\hline Experiment 4 & 9.409 & 13.30 & 10.59 & 13.07 \\
\hline Average & 9.408 & 13.75 & 10.34 & 13.51 \\
\hline Std. Dev. & 0.0266 & 0.425 & 0.240 & 0.620 \\
\hline
\end{tabular}

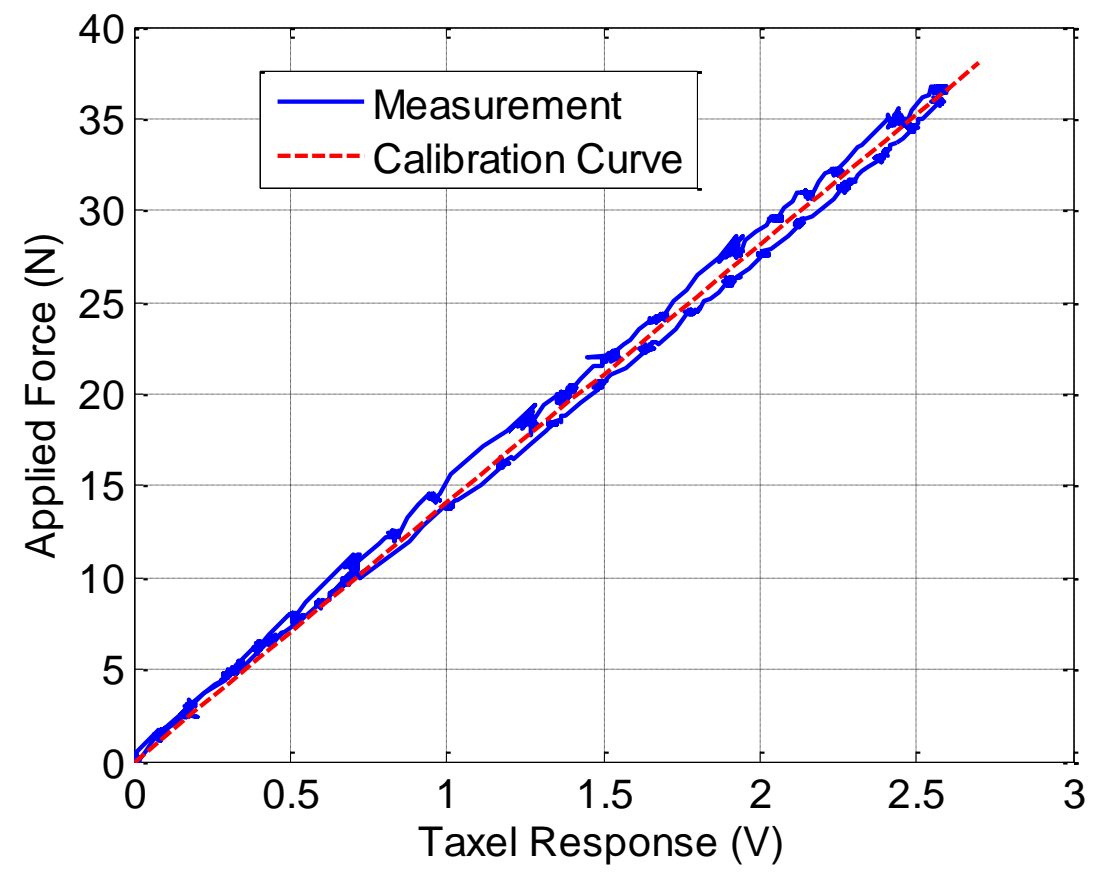

Figure 3-19 Calibration curve fitted from measurements of a single dataset on one taxel 


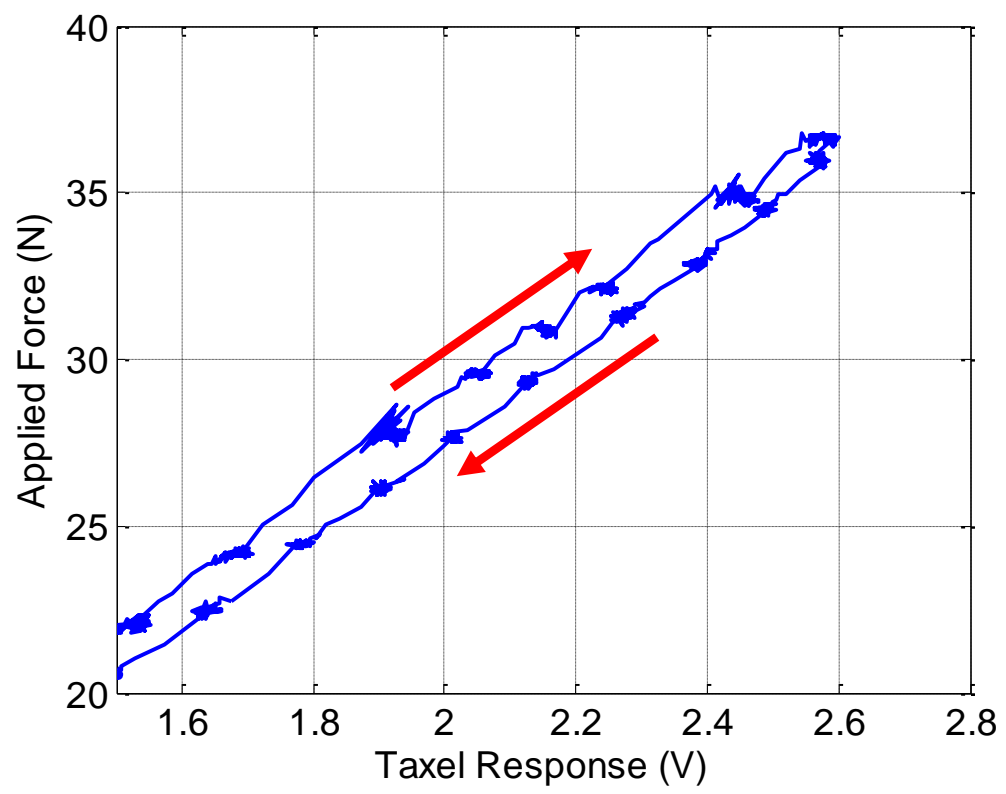

Figure 3-20 Calibration curve (close-up) from single taxel showing hysteresis of response

From the same tests that were used to calibrate each taxel, we can also determine a rough estimate of the Young's modulus of each taxel. The Young's modulus describes the rate of change of the reaction force of elastic materials to strain. The Young's modulus of each taxel was calculated by fitting a linear equation to the plot of applied force as a function of deformation (figure 3-21). Note the y-intercept at zero deformation is not zero for these fits. A polynomial fit or other equation can be used to better model the behavior of the taxel force response. Four our purposes only the linear fit was used to show the slope consistency between taxels. Table 3-2 contains slope values for all linear fits of the four taxels tested. The variation between taxels is approximately 6-7\%, much lower than the variation in the calibration curves.

Hysteresis is also present in the Young's modulus plots and shown in figure 3.22. The nonlinearities and hysteresis present in the taxel can be ignored if, for future tests, we want to model the taxel as a linear spring. 
Table 3-2 Slope values calculated from curves of taxel deformation vs. applied force

\begin{tabular}{|c|c|c|c|c|}
\hline Units: $k N / m$ & Taxel 1 & Taxel 2 & Taxel 3 & Taxel 4 \\
\hline Experiment 1 & 229.6 & 219.4 & 210.7 & 228.0 \\
\hline Experiment 2 & 231.6 & 219.0 & 227.7 & 224.7 \\
\hline Experiment 3 & 241.4 & 221.7 & 227.0 & 228.1 \\
\hline Experiment 4 & 235.7 & 221.8 & 228.4 & 227.6 \\
\hline Average & 234.6 & 220.5 & 223.4 & 227.1 \\
\hline Std. Dev. & 4.49 & 1.29 & 7.39 & 1.41 \\
\hline
\end{tabular}

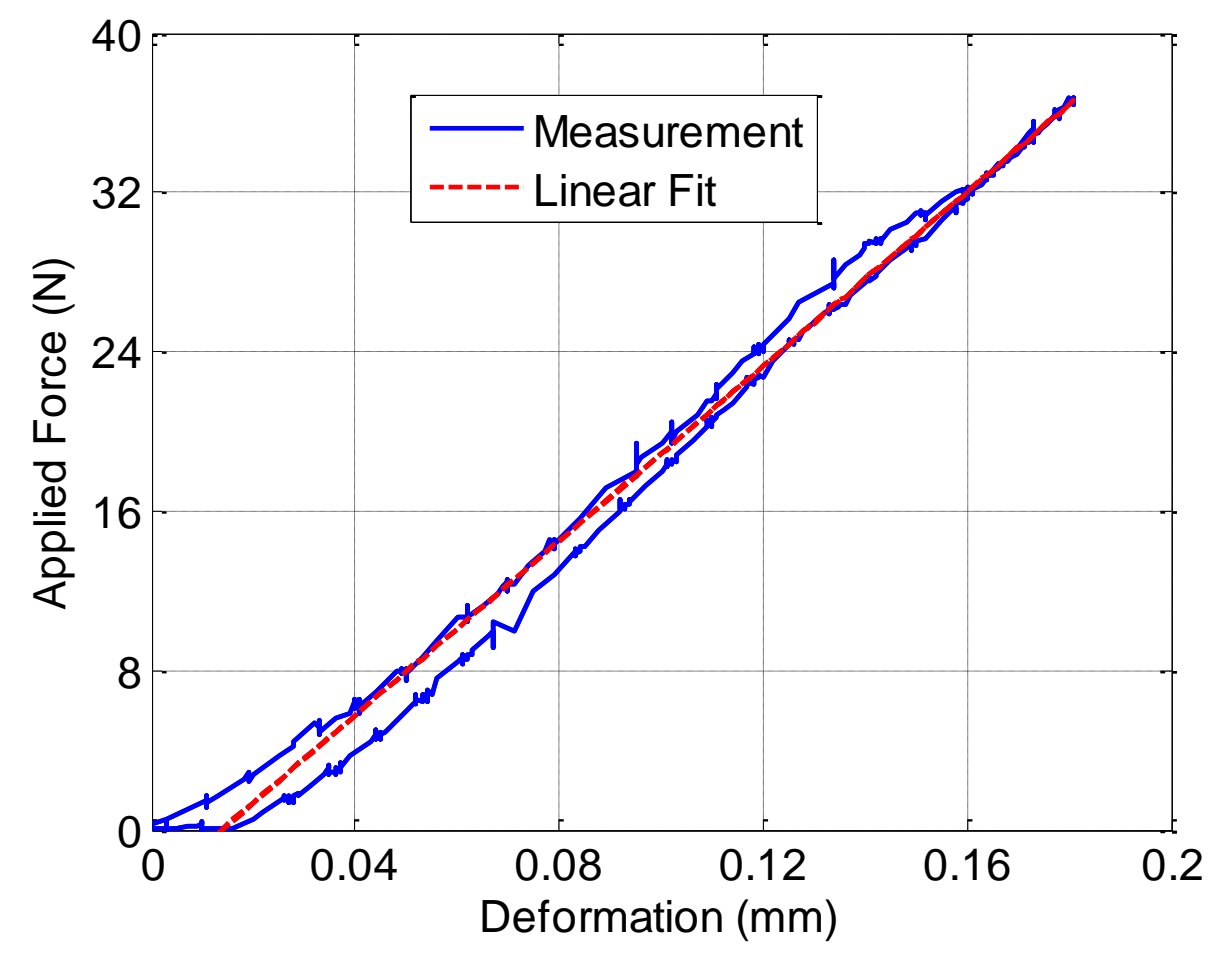

Figure 3-21 Linear fit of force response of single taxel to deformation 


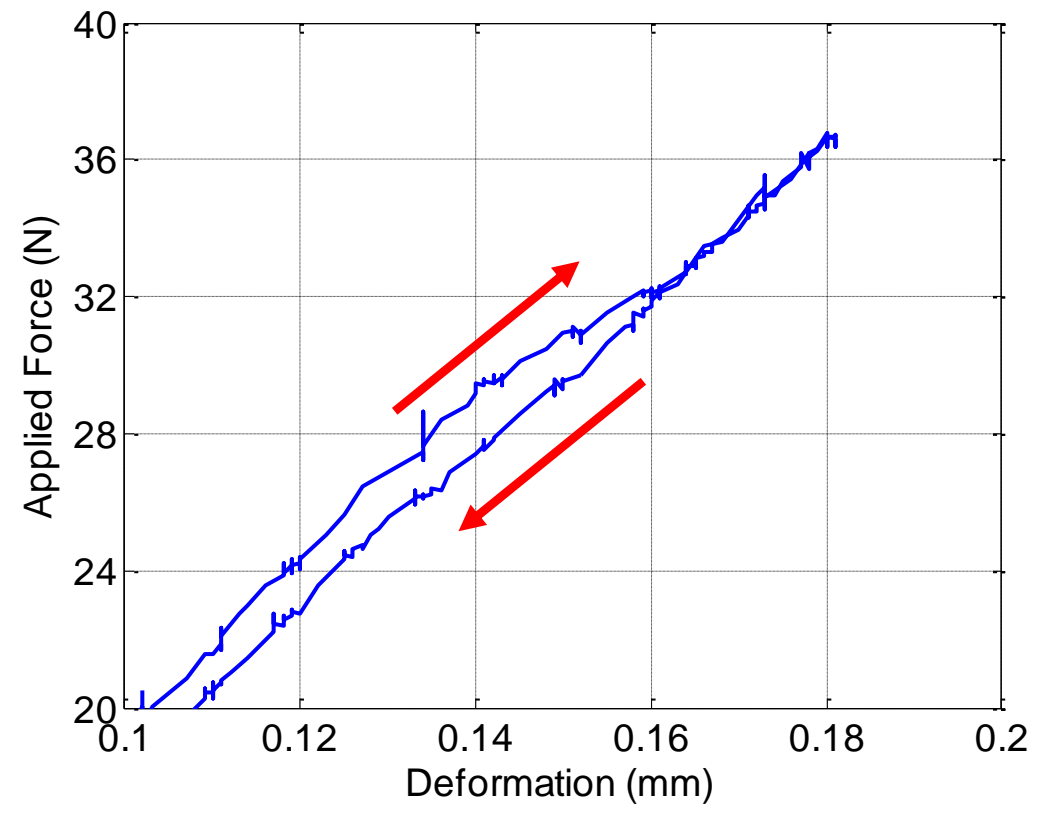

Figure 3-22 Hysteresis in force response of single taxel to deformation

\subsubsection{TTI Load Cell}

The MLP-25 TTI load cell is a strain gauge based transducer. Strain gauges take advantage of conductive materials that experience change in resistance due to deformation. When a load is applied to the load cell the surface that the strain gauge is fixed to deforms. The change in resistance caused by the deformation is measured by a Wheatstone bridge and then related to a force through signal conditioning and calibration. The load cell in our experiment was shipped pre-calibrated - this was validated through testing with a known weight. The load cell has a 25lb capacity.

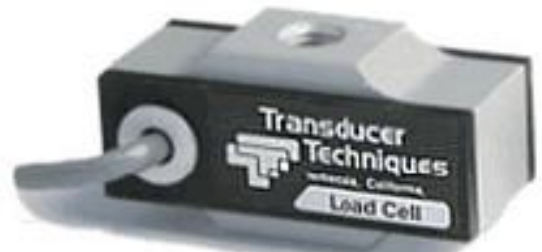

Figure 3-23 TTI MLP-25 load cell 
The load cell has the following specifications [46]:

- Nonlinearity: $0.1 \%$ of rated output $(\mathrm{RO})$

- Hysteresis: $0.1 \%$ of $\mathrm{RO}$

- Nonrepeatability: $0.05 \%$ of $\mathrm{RO}$

- Zero Balance: $1.0 \%$ of RO

The change in resistance of the strain gauge during loading is measured by a TTI TMO-1 load cell signal conditioner. The TMO-1 has a $2200 \mathrm{~Hz}$ bandwidth filter. The TMO-1 must be zeroed with a multimeter with zero load applied to the load cell. A gain potentiometer is used with the load cell calibration chart (shipped with item) to adjust the response of the signal conditioner. The TMO-1 requires a 12VDC power source and outputs $0-8 \mathrm{~V}$. The output signal is wired to the analog input module of the cRIO.

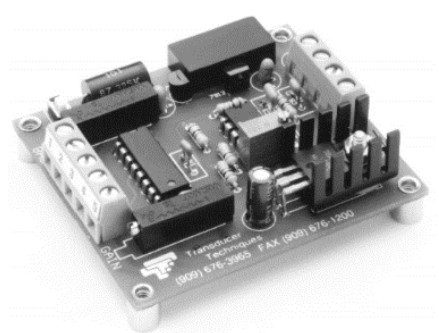

Figure 3-24 TTI TMO-1 signal conditioner

The $0-8 \mathrm{~V}$ analog output signal from the signal conditioner corresponds with an applied force on the load cell of 0-25lbs. As previously stated, the cRIO analog input module has a resolution of $4.88 \mathrm{mV}$. Thus, the sensing resolution of the load cell for our system is $0.07 \mathrm{~N}$.

The load cell was mounted on the linear actuator with a 3D printed mounting plate designed in CAD software. The material of the mounting plate is Somos $\circledast$ WaterShed XC11122. The Young's modulus of cured XC11122 is approximately 2750MPa [47]. 
The cylindrical plunger was machined from a $12 \mathrm{~mm}$ diameter 6060 aluminum rod. One end of the plunger was threaded so it could be connected to the load cell with an adapter. The other end was machined down to a diameter of $9.9 \mathrm{~mm}$ to be the same size as the sensing area of the taxels.

(a)

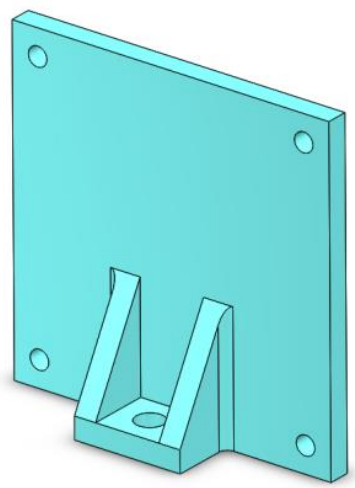

Figure 3-25 (a) 3D printed mounting plate \& (b) cylindrical load applicator

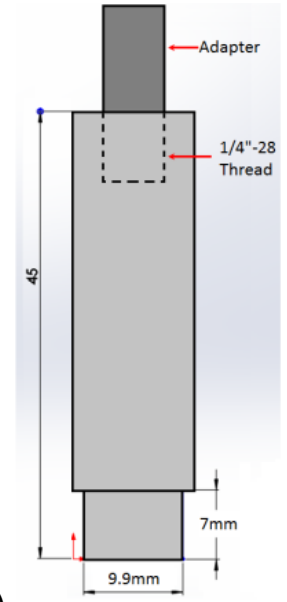

(b)

\subsection{Fabricated Silicone Skin}

Skin samples made from two different materials (silicone and Frubber®) were tested in this thesis. With the exception of one sample, all of the silicone skin patches were fabricated specifically for this thesis at UTARI. The Frubber® samples were fabricated by David Hanson. In this section is detailed the fabrication process of silicone rubber skin.

All of the silicone skin patches created for this thesis were made at UTARI from P-10 silicone rubber from Silicones, Inc. The RTV silicone rubber is a 10:1 mixture of base to curing agent. Each skin sample was made from a 3D-printed mold that was parylene-coated in a SCS parylene deposition system. The ultra-thin $(5 \mu \mathrm{m})$ parylene coating on the 3D-printed mold helps with removal of the cured silicone. The skin samples were made into $40 \mathrm{~mm} \times 40 \mathrm{~mm}$ squares of $2 \mathrm{~mm}, 4 \mathrm{~mm}$, and $6 \mathrm{~mm}$ thickness. In 
addition, silicone skin samples were made into $40 \mathrm{~mm} \times 60 \mathrm{~mm}$ rectangles with $4 \mathrm{~mm}$ thickness. Once mixed and poured into the molds, the silicone mixture is degassed in a vacuum chamber, removing air bubbles introduced into the silicone during mixture. Once most of the air was removed (approximately 30 minutes) the molds were covered in a flat plate and cured inside an environmental oven. Curing in an oven is not necessary except for convenience; the silicone will cure at room temperature in less than 24 hours. Once cured, P-10 silicone rubber has a tensile strength of $2413 \pm 345 \mathrm{kPa}$ and percent elongation of $450 \pm 50$.

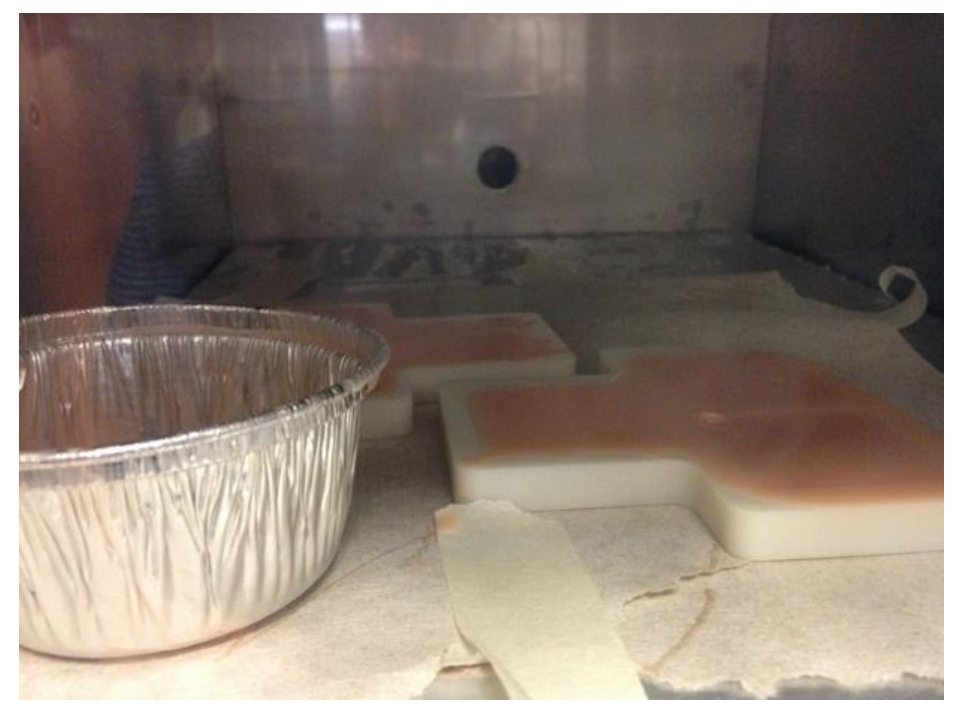

Figure 3-26 RTV silicone in 3D printed molds during degassing 
Chapter 4

\section{Robot Skin Experiments \& Models}

\subsection{Soft Skin Material Testing}

If social robots are to be covered in soft skin, understanding the behavior of the material used for skin is important. Many different materials can be used for the soft tissue on robot skin. In this thesis we looked at two types of material: Frubber® and silicone rubber.

Both silicone rubber and Frubber ${ }^{\circledR}$ experience hysteresis and drift. Hysteresis is a nonlinear phenomenon where the relationship between a system's input and output differ between loading and unloading [5]. Drift occurs when a material relaxes after a constant strain input and the stress decreases with time. To study hysteresis and drift in silicone rubber and Frubber ${ }^{\circledR}$ we applied several motion inputs to soft skin samples without an embedded taxel. The load applicator descended into each sample while the deformation and reaction force were recorded.

Figure 4-1 and figure 4-2 show the hysteresis present in a $5 \mathrm{~mm}$ thick silicone rubber sample and a $6 \mathrm{~mm}$ thick Frubber ${ }^{\circledR}$ sample, respectively. The silicone rubber had a maximum difference between loading and unloading of approximately $15 \%$; for Frubber ${ }^{\circledR}$ the maximum difference was approximately $55 \%$. The force response of silicone rubber skin to strain is approximately linear while the Frubber ${ }^{\circledR}$ response is highly nonlinear. It is obvious the silicone rubber is much stiffer than the Frubber® sample. The silicone rubber reached a reaction force of $10 \mathrm{~N}$ after only $16 \%$ deformation; the Frubber ${ }^{\circledR}$ sample required $70.8 \%$ deformation to reach $10 \mathrm{~N}$. The rate dependence of the force response in silicone rubber was tested and is small but measureable (figure 41). As the strain rate increases the stress-strain curve shifts towards the left, meaning at higher strain rates an equivalent deformation produces higher stress than at lower strain 
rates. This could be explained by the material having less time to relax at higher strainrates.

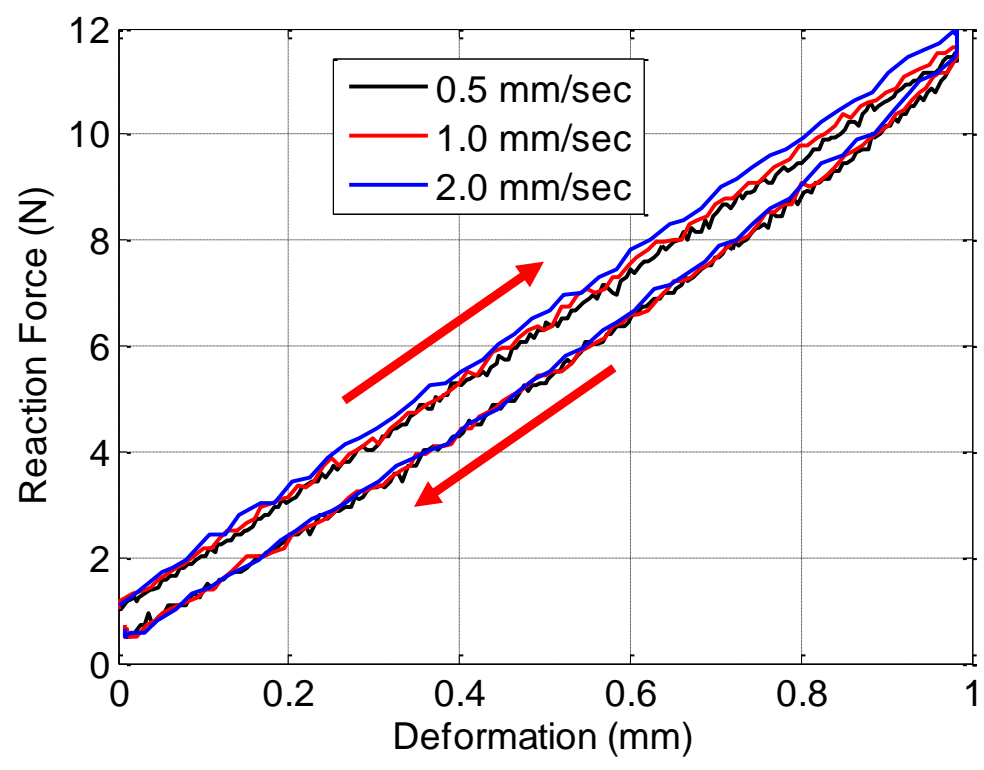

(a)

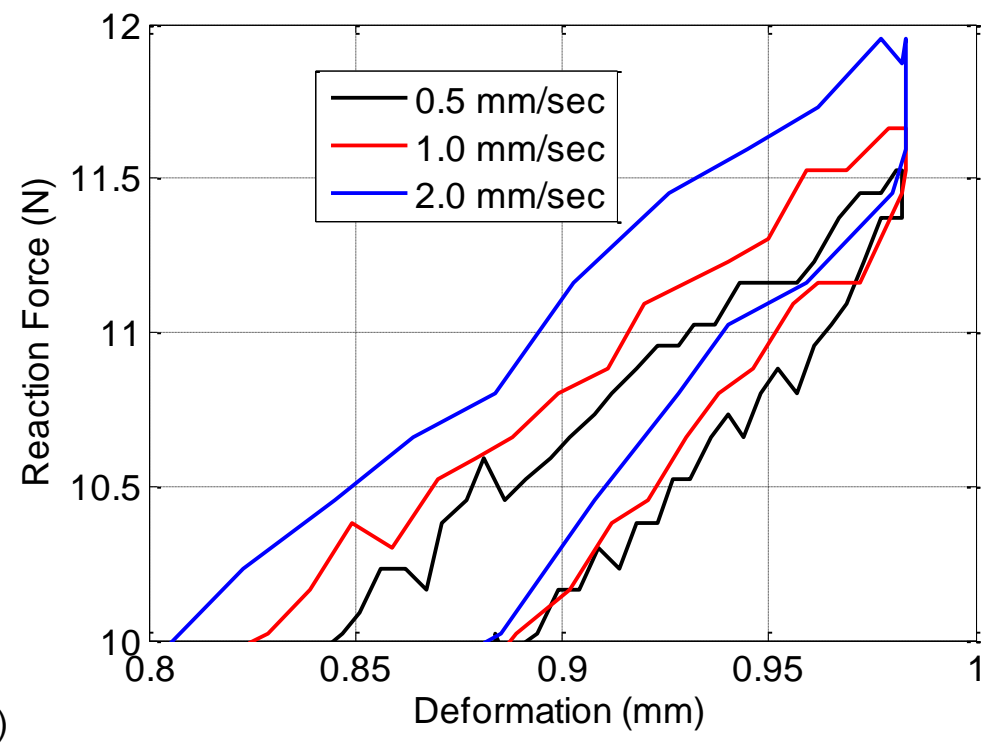

Figure 4-1 (a) Force response of $5 \mathrm{~mm}$ silicone skin sample to deformation. The rate dependence (b) of the material response is small but measureable 

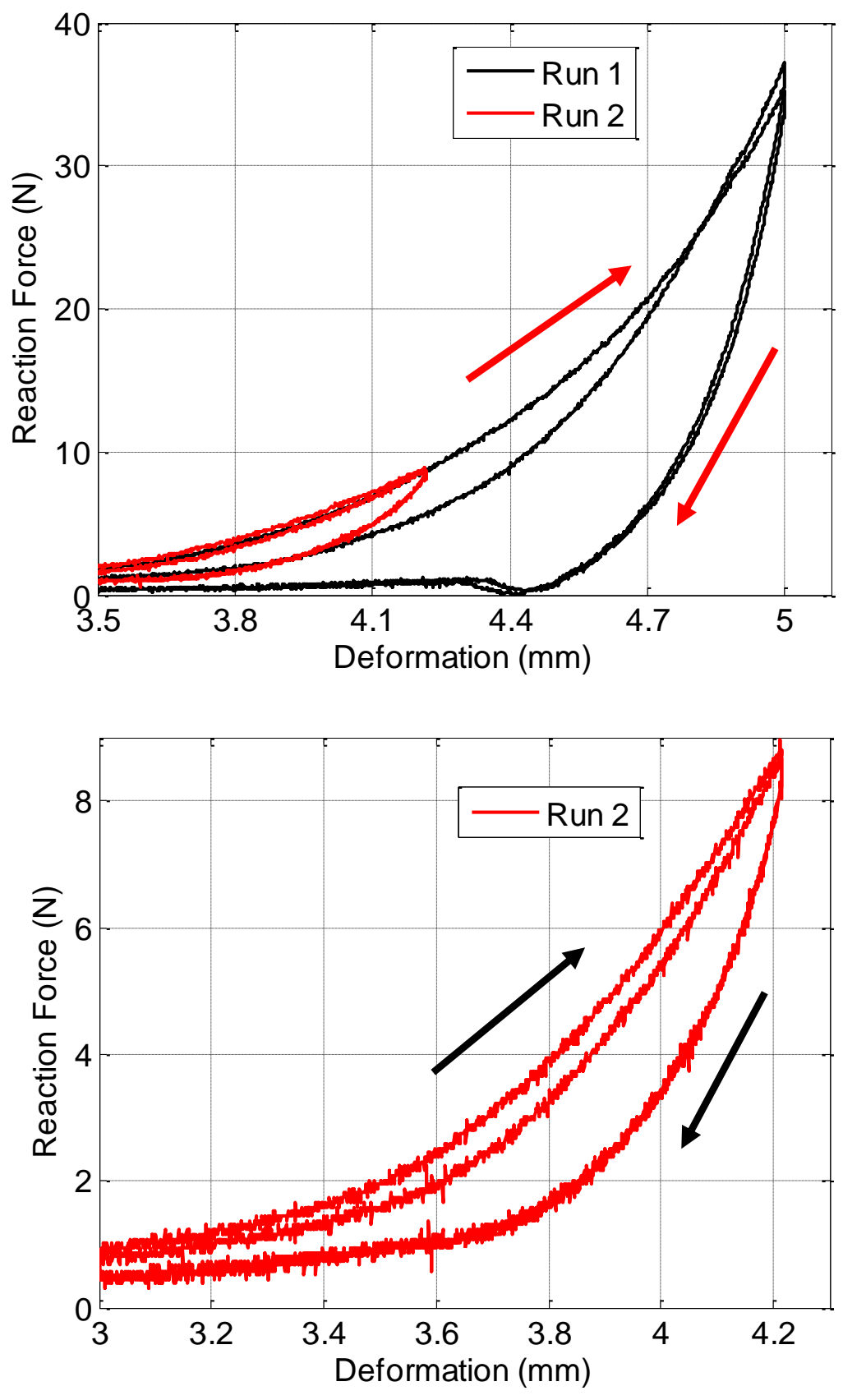

Figure 4-2 (a) Force response of $6 \mathrm{~mm}$ Frubber ${ }^{\circledR}$ skin to deformation; (b) close-up of Run 
Hysteresis models been described in literature and include models such as the Bouc-Wen model and Preisach model [5] [36]. The Bouc-Wen model has been used to describe such hysteretic systems as magnetic materials [5]. In the future, a Preisach model will be implemented on a silicone rubber sample to mimic the behavior of a test case. The Preisach model is useful because its parameters can be identified from reference curves gained through experiment [36].

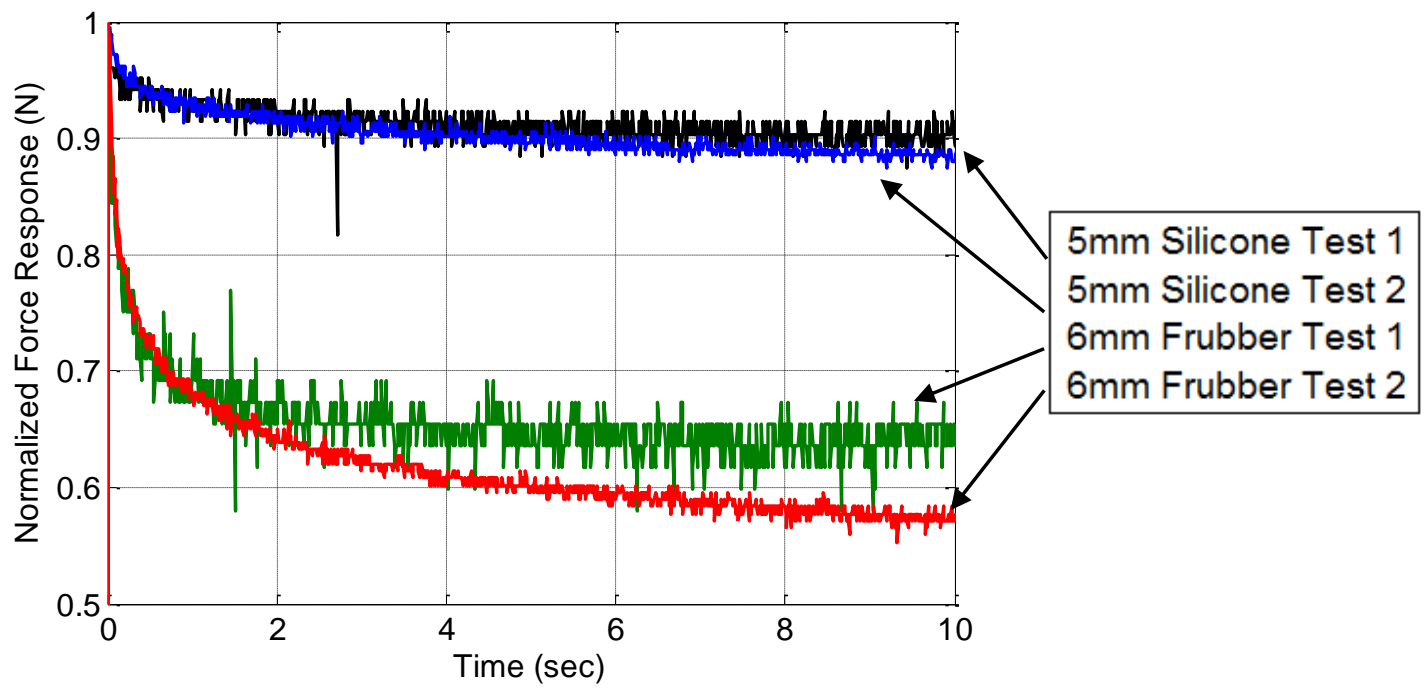

Figure 4-3 Normalized stress response of $5 \mathrm{~mm}$ silicone rubber $\& 6 \mathrm{~mm}$ Frubber ${ }^{\circledR}$

Drift response of both samples is shown in figure 4.3. The graph shows the normalized force response of each sample over time so that the two materials can be better compared. As can be seen from the graph, the Frubber ${ }^{\circledR}$ sample experiences higher stress relaxation compared to the silicone rubber. After ten seconds the silicone force response had decreased by $10.6 \%$ for test 1 and $12.0 \%$ for test 2 for an average of 11.3\%. After ten seconds the Frubber® force response had decreased by $39.3 \%$ for test 1 and $42.3 \%$ for test 2 for an average of $40.8 \%$. The initial force response immediately 
after the step input for each case was: $7.45 \mathrm{~N}$ for silicone test $1 ; 13.1 \mathrm{~N}$ for silicone test 2 ; 3.74N for Frubber® test $1 ; 11.66 \mathrm{~N}$ for Frubber® test 2.

\subsection{Taxel Placement Testing}

Robot sensing taxels can be placed above, below or inside soft skin. We performed two different tests to study the effect of this design change to the skin response: in one test we placed a taxel underneath a $4 \mathrm{~mm}$ silicone skin sample; in the second test we placed the taxel on the surface of the same skin sample. The load applicator was centered directly above the taxel. The same motion profile for the load applicator was used for each case: the load applicator repeatedly depressed $1 \mathrm{~mm}$ into the skin sample. The results for taxel placement on top and on bottom of the skin are seen in figure 7. Both the applied and sensed force were higher with the taxel on top of the skin sample. The max applied load was $34 \mathrm{~N}$ with the taxel on top and $15 \mathrm{~N}$ with the taxel on the bottom. The max sensed load was $18.5 \mathrm{~N}$ with the taxel on top and $8 \mathrm{~N}$ with the taxel on bottom.

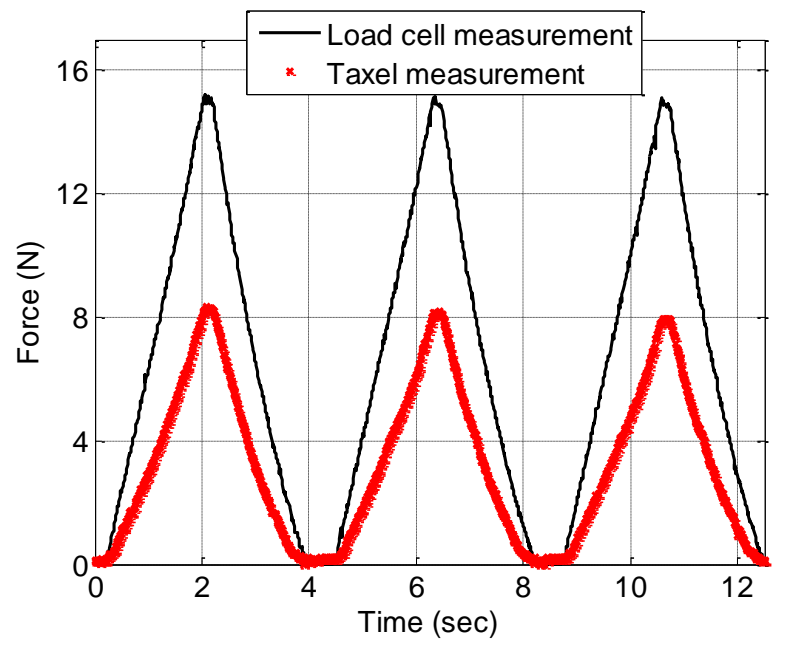

Figure 4-4 Force measured by load cell \& taxel with taxel underneath $4 \mathrm{~mm}$ silicone 


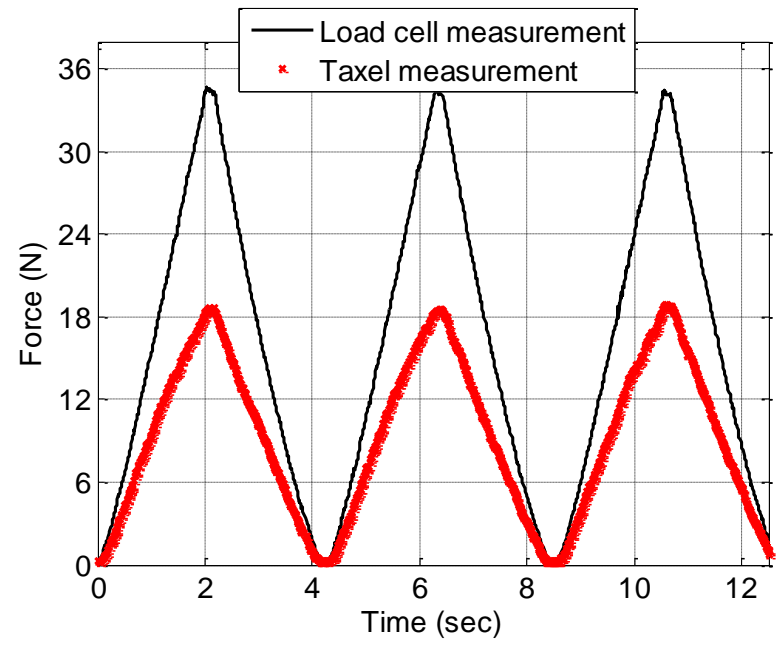

Figure 4-5 Force measured by load cell \& taxel with taxel on top of $4 \mathrm{~mm}$ silicone 4.3 Robot Skin with Embedded Taxels Testing

\subsubsection{Parameter Identification for Reduced-Order Model}

A primary goal of the testbed was to relate the input force on a skin sample to the force detected by an embedded taxel in the sample. This relationship describes the difference between what a robot is subjected to in an environment (the applied force to robot skin) and what the robot perceives during an interaction (the sensed force by robot skin). This interaction between robot skin and environment is what we have designed our testbed to mimic (figure 4-6). The load applicator, centered above a taxel, applies a uniform load on a small area which is then distributed through soft skin to the taxel below and adjacent areas (including adjacent taxels). During real implementation of robot skin, there will exist a large array of taxels detecting signals all over the surface of the robot. The effect of an applied force to adjacent taxels is studied in section 4.3 .2 of this thesis.

The models relating input force on robot skin to the force detected by an embedded taxel are required for robot skin implementation in SkinSim. Because of the nature of SkinSim, a simulation environment meant to optimize numerous parameters of 
large-scale robot skin, a scalable reduced-order model of the force to force relationship is desired. While a force to deformation relationship in robot skin may be non-linear, it is not unreasonable to suggest a linear relationship between applied force and sensed force. This is showcased in figures 4-7 and 4-8. A linear reduced-order model describing the relationship between applied force and sensed force will serve well for the purposes of SkinSim, where an appropriate scalable approximation can be supplanted for a complicated, computationally expensive model.

A linear reduced-order "dispersion" model was developed to relate the force applied to robot skin to the sensed force by a taxel underneath the skin (figure 4-9) [4]. The model uses the term "dispersion" to describe it because the applied force is dispersed throughout the material. The linear model consists of one mass element connected to a tactile sensor by two springs and two dashpots. The total force applied $F_{L C}$ is contributed to by both springs and both dashpots while the taxel sensed force $F_{F F}$ is only contributed to by spring $k_{1}$ and dashpot $b_{1}$. The spring $k_{2}$ and dashpot $b_{2}$ represent the force dispersed throughout the soft robot skin not detected by the taxel.

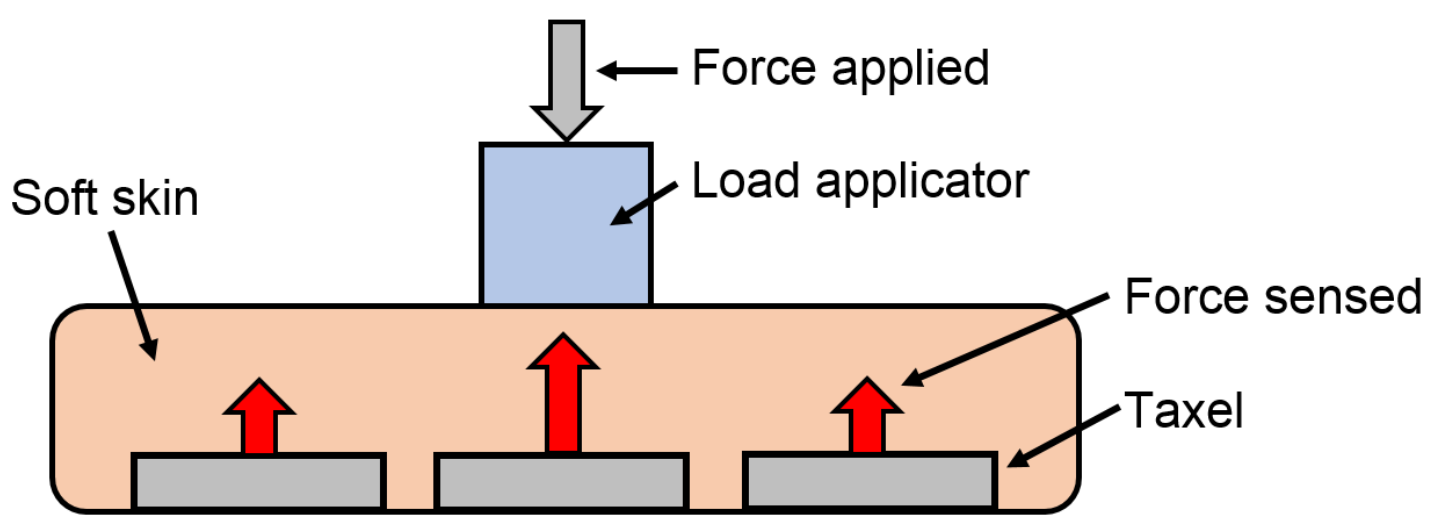

Figure 4-6 Robot skin with embedded taxels 


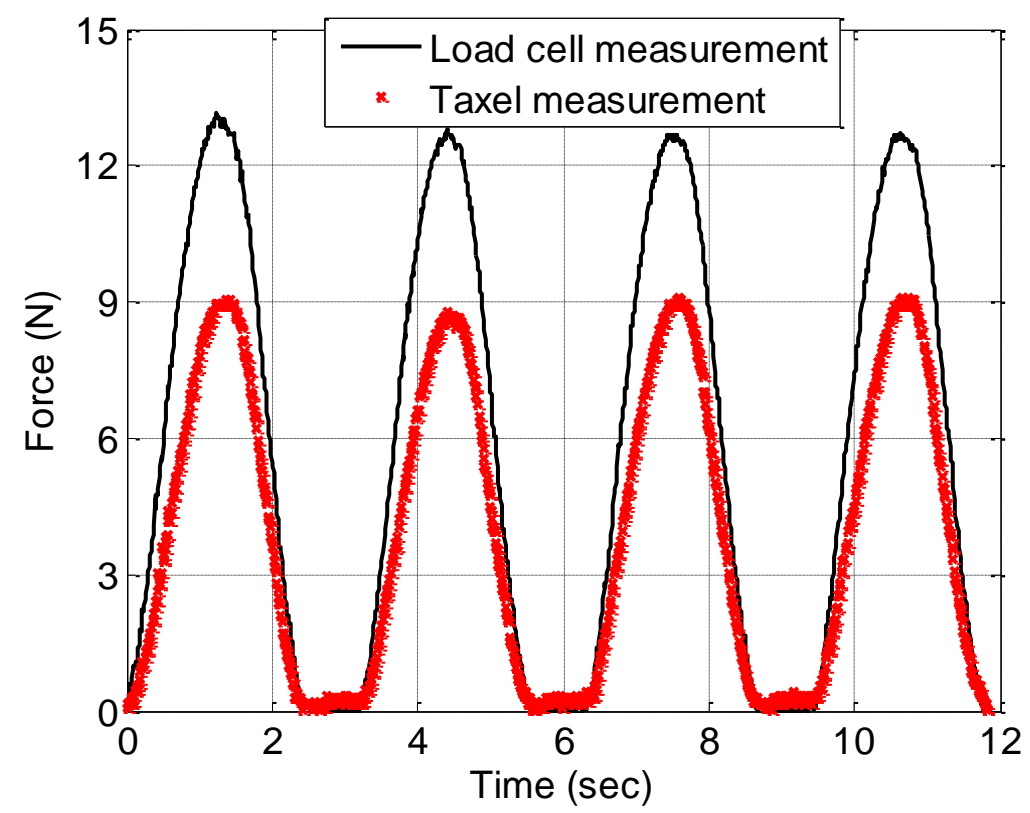

Figure 4-7 Force response of load cell \& taxel with $4 \mathrm{~mm}$ silicone to cyclic deformation

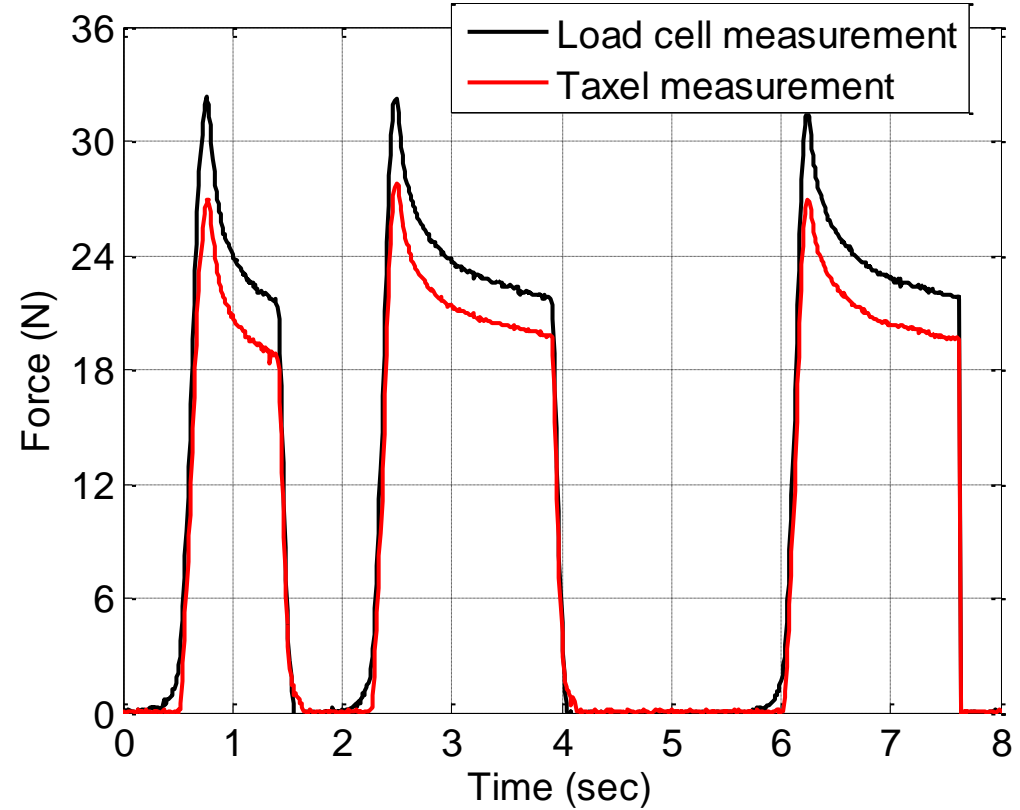

Figure 4-8 Force response of load cell \& taxel with $6 \mathrm{~mm}$ Frubber ${ }^{\circledR}$ to step-inputs 


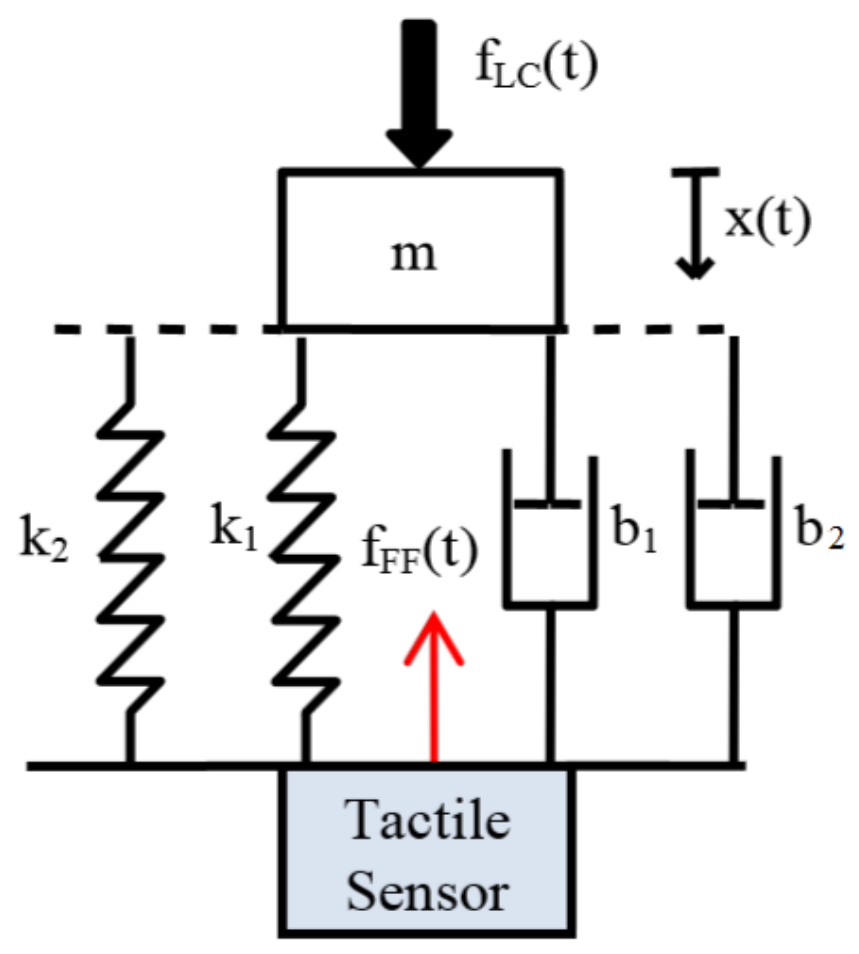

Figure 4-9 Reduced-order "dispersion" model relating applied force to sensed force in robot skin

The two forces $F_{L C}$ and $F_{F F}$ are related through the following equations:

$$
\begin{gathered}
F_{L C}(t)=m(\ddot{x}(t)-g)+\left(b_{1}+b_{2}\right) \dot{x}(t)+\left(k_{1}+k_{2}\right) x(t) \\
F_{F F}=b_{1} \dot{x}(t)+k_{1} x(t)
\end{gathered}
$$

We performed experiments on three different types of robot skin and identified the parameters of the "dispersion" model for each. The robot skin samples consisted of silicone rubber skin with a FlexiForce taxel underneath the skin. Three samples each of three different thicknesses of silicone rubber skin were tested, i.e., three samples of $2 \mathrm{~mm}$ skin, three samples of $4 \mathrm{~mm}$ skin and three samples of $6 \mathrm{~mm}$ skin were tested. Further, each individual skin sample was tested three times. After each experiment the parameters $m, k_{1}, b_{1}, k_{2}$, and $b_{2}$ were identified using a linear least squares algorithm. 
The basis functions used in the linear least squares algorithm were those for $F_{L C}$ and $F_{F F}$. Because both of these functions include either $\ddot{x}(t)$ or $\dot{x}(t)$ a differentiable motion input was required for $x(t)$. Towards this end, the load applicator depressed into the robot skin with a sinusoidal motion given by:

$$
x(t)=A \sin (B t)+C
$$

At $x=0$ the load applicator is just at the surface of the robot skin. Positive $x$ values denote compression of the skin. For each parameter identification test four sinusoidal periods were recorded. Different values of $A, B$ and $C$ were used for each thickness of skin, but the same values were used for samples of the same thickness. However, the actual motion of the load applicator measured by the encoder varied from these set values by a small amount. To find the exact values for each experimental motion profile the parameters $\mathrm{A}, \mathrm{B}$ and $\mathrm{C}$ were manually adjusted to minimize the error between the actual measurement position and position calculated using $A, B$ and $C$. An example comparing the measured position to the position calculated using the identified $A, B$ and $C$ is seen in figure 4-10. As seen in the figure, they overlap. The time values were adjusted to minimize the error which is why the plot shows negative values for time $t$. The adjusted values for A, B, C and time $t$ were used in the linear least squares algorithm. A representative plot of the error between the actual position measurements and calculated measurements is seen in figure 4-11. The error is largest at the beginning of the dataset when the linear actuator is starting with zero velocity. Outside the beginning of the motion the maximum error is approximately $0.009 \mathrm{~mm}$. The average error for all measurement sets of all three thicknesses is approximately $0.002-$ $0.006 \mathrm{~mm}$.

Given the prescribed sinusoidal motion input, the basis functions $F_{L C}$ and $F_{F F}$ become: 


$$
\begin{gathered}
F_{L C}(t)=m\left(-A B^{2} \sin (B t)-g\right)+\left(b_{1}+b_{2}\right) A B \cos (B t) \\
+\left(k_{1}+k_{2}\right)(A \sin (B t)+C) \\
F_{F F}=b_{1} A B \cos (B t)+k_{1}(A \sin (B t)+C)
\end{gathered}
$$

The batch of measurements used for the linear least squares algorithm were

$$
\left\{F_{L C_{1}}, F_{F F_{1}}, t_{1} ; F_{L C_{2}}, F_{F F_{2}}, t_{2} ; \ldots ; F_{L C_{m}}, F_{F F_{m}}, t_{m}\right\}
$$

with the following states to be estimated:

$$
x_{1}=m ; x_{2}=b_{1} ; x_{3}=k_{1} ; x_{4}=b_{2} ; x_{5}=k_{2}
$$

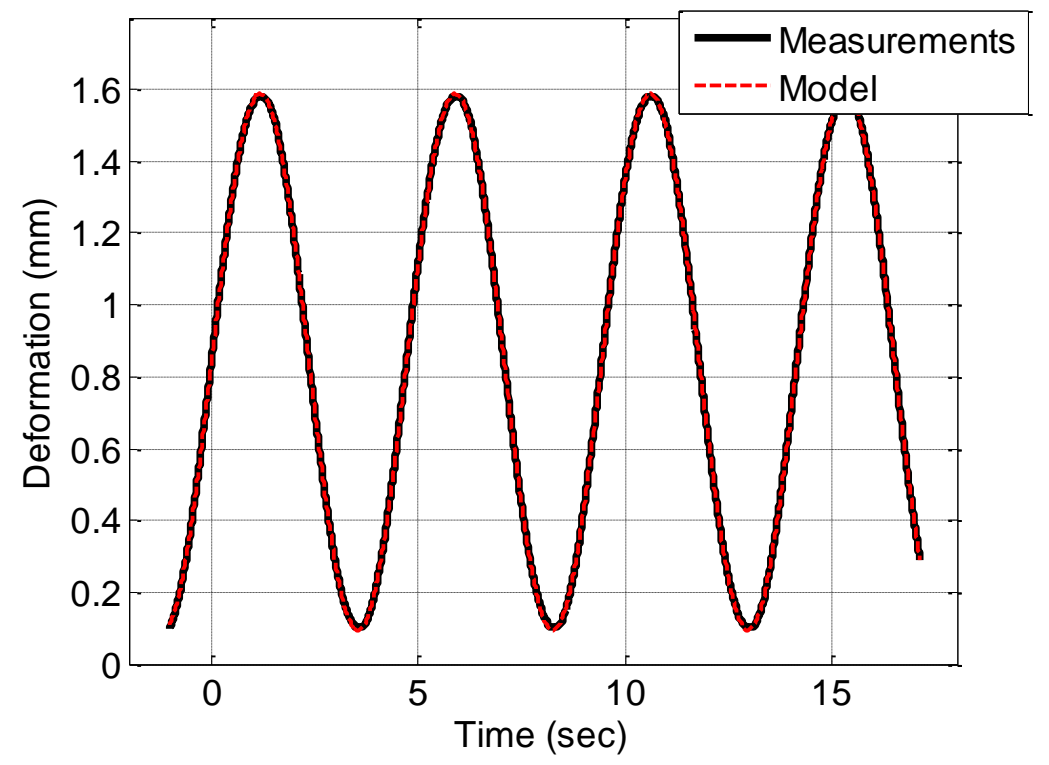

Figure 4-10 Sinusoidal motion measurements vs. calculated measurements

Three samples of each thickness of silicone skin $(2 \mathrm{~mm}, 4 \mathrm{~mm}$ and $6 \mathrm{~mm})$ were fabricated and tested. Each sample was then tested three times except for the $4 \mathrm{~mm} \# 1$ silicone sample which was only tested once. The conditions for testing were the same for each sample of the same thickness. 


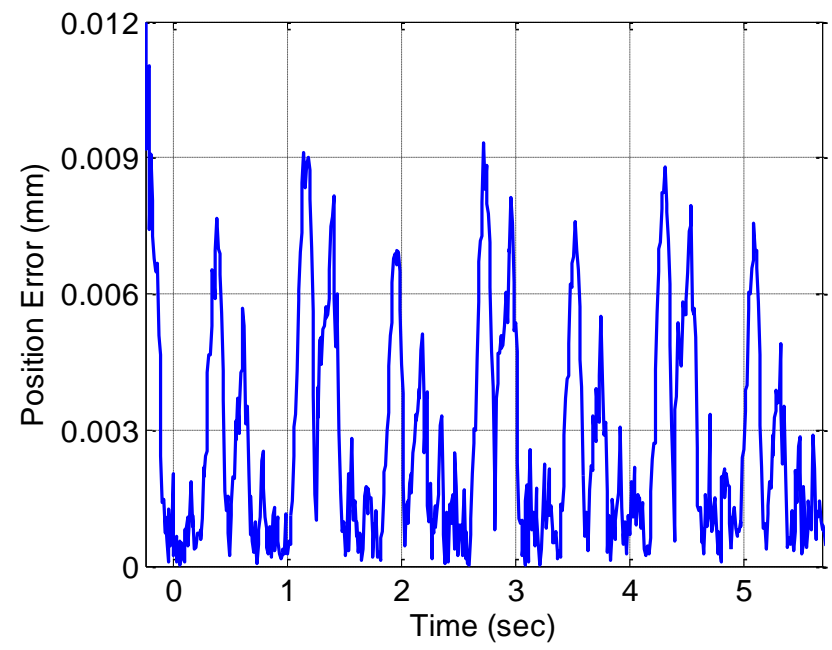

Figure 4-11 Position error between measurements \& model for $6 \mathrm{~mm}$ silicone skin sample

The average values of the identified parameters for each sample of skin is seen

in Table 4-1, Table 4-2 and Table 4-3. Table 4-4 shows the average value of the identified parameters for each thickness of skin.

Table 4-1 Average values of identified parameters of "dispersion" model for $2 \mathrm{~mm}$ silicone

\begin{tabular}{|c|c|c|c|}
\hline & $2 \mathrm{~mm} \# 1$ & $2 \mathrm{~mm} \# 2$ & $2 \mathrm{~mm} \# 3$ \\
\hline m (kg) & 0.281 & 0.247 & 0.282 \\
\hline std. dev. (kg) & 0.028 & .003 & 0.014 \\
\hline b1 (kg/s) & 1464.4 & 1286.1 & 1242.1 \\
\hline std. dev. $(\mathrm{kg} / \mathrm{s})$ & 65.1 & 99.5 & 35.4 \\
\hline k1 (N/m) & 27635 & 22738 & 24753 \\
\hline std. dev. $(\mathrm{N} / \mathrm{m})$ & 3174 & 379 & 1087 \\
\hline b2 (kg/s) & 645.5 & 575.5 & 572.8 \\
\hline std. dev. $(\mathrm{kg} / \mathrm{s})$ & 21.4 & 48.0 & 33.2 \\
\hline k2 (N/m) & 8879 & 8285 & 8694 \\
\hline std. dev. $(\mathrm{N} / \mathrm{m})$ & 757 & 422 & 445 \\
\hline
\end{tabular}

Table 4-2 Average values of identified parameters of "dispersion" model for $4 \mathrm{~mm}$ silicone

\begin{tabular}{|c|c|c|c|}
\hline & $\mathbf{4 m m}$ \#1 & $\mathbf{4 m m ~ \# 2}$ & $\mathbf{4 m m ~ \# 3}$ \\
\hline $\mathbf{m ~ ( k g )}$ & 0.132 & 0.247 & 0.204 \\
\hline std. dev. $(\mathrm{kg})$ & NA & .011 & .051 \\
\hline $\mathbf{b 1} \mathbf{( k g / s )}$ & 843.1 & 1186.2 & 725.1 \\
\hline std. dev. (kg/s) & NA & 86.0 & 113.8 \\
\hline
\end{tabular}


Table 4-2-Continued

\begin{tabular}{|c|c|c|c|}
\hline $\mathbf{k 1} \mathbf{( N / \mathbf { m } )}$ & 8061 & 10382 & 9375 \\
\hline std. dev. (N/m) & NA & 770 & 299 \\
\hline $\mathbf{b 2} \mathbf{( k g / s )}$ & 772.8 & 662.1 & 563.6 \\
\hline std. dev. $(\mathrm{kg} / \mathrm{s})$ & NA & 65.4 & 13.6 \\
\hline $\mathbf{k 2} \mathbf{( N / \mathbf { m } )}$ & 5563 & 5605 & 5167 \\
\hline std. dev. (N/m) & NA & 50.3 & 396 \\
\hline
\end{tabular}

Table 4-3 Average values of identified parameters of "dispersion" model for $6 \mathrm{~mm}$ silicone

\begin{tabular}{|c|c|c|c|}
\hline & $\mathbf{6 m m ~ \# 1}$ & $\mathbf{6 m m ~ \# 2}$ & $\mathbf{6 m m ~ \# 3 ~}$ \\
\hline $\mathbf{m ~} \mathbf{( k g})$ & 0.199 & 0.180 & 0.222 \\
\hline std. dev. (kg) & 0.029 & .017 & 0.006 \\
\hline $\mathbf{b 1} \mathbf{( k g / s )}$ & 202.5 & 164.6 & 378.5 \\
\hline std. dev. (kg/s) & 33.7 & 31.2 & 17.0 \\
\hline $\mathbf{k 1} \mathbf{( N / \mathbf { m } )}$ & 4930 & 4222 & 3862 \\
\hline std. dev. (N/m) & 61.0 & 209 & 209 \\
\hline $\mathbf{b 2} \mathbf{( k g / s )}$ & 710.7 & 696.7 & 900.5 \\
\hline std. dev. (kg/s) & 37.7 & 54.6 & 45.6 \\
\hline $\mathbf{k 2} \mathbf{( N / \mathbf { m } )}$ & 7652 & 7186 & 6968 \\
\hline std. dev. (N/m) & 8.77 & 94.6 & 222 \\
\hline
\end{tabular}

Table 4-4 Average values of identified parameters of "dispersion" model for each

thickness of silicone skin

\begin{tabular}{|c|c|c|c|}
\hline & 2mm Skin & 4mm Skin & 6mm Skin \\
\hline m (kg) & 0.270 & 0.212 & 0.200 \\
\hline std. dev. (kg) & 0.016 & .051 & .018 \\
\hline b1 (Ns/m) & 1330 & 939.5 & 248.6 \\
\hline std. dev. $(\mathrm{kg} / \mathrm{s})$ & 96.0 & 236 & 93.1 \\
\hline k1 (N/m) & 25042 & 9619 & 4338 \\
\hline std. dev. $(\mathrm{N} / \mathrm{m})$ & 2010 & 957 & 444 \\
\hline b2 (Ns/m) & 598.0 & 635.7 & 769.3 \\
\hline std. dev. $(\mathrm{kg} / \mathrm{s})$ & 33.7 & 84.4 & 92.9 \\
\hline k2 (N/m) & 8619 & 5411 & 7269 \\
\hline std. dev. $(\mathrm{N} / \mathrm{m})$ & 248 & 336 & 285 \\
\hline
\end{tabular}

The mass $m$ for every identified case is below $0.300 \mathrm{~kg}$. This can be attributed to acceleration of the deformation having very little effect on the applied or sensed force.

The same can be said of the damping factor $b_{1}$ and $b_{2}$ which are all roughly an order of 
magnitude smaller than $k_{1}$ and $k_{2}$ for each sample. The dominant factor influencing $F_{L C}$ and $F_{F F}$ in all cases are the spring constants $k_{1}$ and $k_{2}$. This means our reduced-order models are predominantly affected by changes in deformation of the robot skin for low frequency motion inputs.

There is significant variation between parameter values for different skin samples of the same thickness. This can be attributed to the variation in composition of each skin sample which can occur during fabrication. The skin samples are from multiple fabrication batches which contain slight differences in composition. The inclusion of anisotropic air bubbles not vacuumed out during degassing also can affect the identified parameters. While the state values differ, all state values from the same thickness of skin share the same order of magnitude. These values show a general range for what can be expected from silicone skin of various thickness when using the "dispersion" model.

A problem encountered with using this model can be seen when looking at the values in Table 4-4. For each thickness of skin, there is not a clear trend in the estimated states. For example, we would expect the value of the mass to consistently increase with thickness. Instead, we see that mass actually decreases (however slightly) as thickness increases from $2 \mathrm{~mm}$ to $6 \mathrm{~mm}$. This does not make physical sense. In addition, there is not a clear trend found when looking at the value $k_{2}$. Unlike $k_{1}$, which continually decreases with increasing skin thickness, $k_{2}$ first decreases and then increases. This irregular change in $k_{2}$ does not lend itself to a tie-in with physical reality. Further, the goal of identifying the parameters is to create some relationship with skin thickness so that many different thicknesses of skin can be implemented in SkinSim. These problems present in this version of the "dispersion" model and parameter identification must be reapproached. 
One approach is to remove the mass as a state that needs to be estimated. Because we know the density of the skin, we can calculate the mass and set it in the "dispersion" model as a constant for each experiment (mass increases as skin thickness increases). We will assume the $\mathrm{m}$ in the "dispersion" model represents a block of skin directly above the taxel. This block of skin has the same dimensions as the sensing area of the taxel and has a height of whatever thickness of skin is being tested. The mass for each thickness was calculated based upon the density of silicone material and was $0.216 \mathrm{~g}, 0.432 \mathrm{~g}$ and $0.648 \mathrm{~g}$ for the $2 \mathrm{~mm}, 4 \mathrm{~mm}$ and $6 \mathrm{~mm}$ model, respectively. With the mass removed as a state, we can rerun the linear least squares algorithm with all the same experiments as before and estimate the parameters $b_{1}, k_{1}, b_{2}$ and $k_{2}$ again. The results of this are seen in Table 4-5. The values of $b_{1}$ and $k_{1}$ remain the same while $b_{2}$ and $k_{2}$ change. While both $b_{2}$ and $k_{2}$ change from the original values, $b_{2}$ only changes slightly. The values of $b_{2}$ decrease by an average of $8.4 \mathrm{Ns} / \mathrm{m}$. The average values of $k_{2}$ decrease very differently for each thickness as follows: $7016 \mathrm{~N} / \mathrm{m}$ for the $2 \mathrm{~mm}$ skin; 2645 $\mathrm{N} / \mathrm{m}$ for the $4 \mathrm{~mm}$ skin; $1669 \mathrm{~N} / \mathrm{m}$ for the $6 \mathrm{~mm}$ skin.

With the new values of the estimated parameters we can identify linear trends for each. Each estimated parameter is plotted as a function of skin thickness for the adjusted "dispersion" model in figures $4-12$ and $4-13$. Both $b_{1}$ and $k_{1}$ decrease rapidly with increasing skin thickness while $b_{2}$ and $k_{2}$ increase gradually with increasing skin thickness.

Table 4-5 Average values of identified parameters of adjusted "dispersion" model for each thickness of silicone skin

\begin{tabular}{|c|c|c|c|}
\hline & 2mm Skin & 4mm Skin & 6 mm Skin \\
\hline b1 (Ns/m) & 1330 & 939.5 & 248.6 \\
\hline std. dev. (kg/s) & 96 & 236 & 93.1 \\
\hline $\mathbf{k 1} \mathbf{( N / \mathbf { m } )}$ & 25042 & 9619 & 4338 \\
\hline std. dev. (N/m) & 2010 & 957 & 444 \\
\hline
\end{tabular}


Figure 4-5-Continued

\begin{tabular}{|c|c|c|c|}
\hline b2 (Ns/m) & 590.2 & 629.2 & 758.3 \\
\hline std. dev. $(\mathrm{kg} / \mathrm{s})$ & 66.7 & 79.1 & 93.9 \\
\hline k2 (N/m) & 1603 & 2766 & 5600 \\
\hline std. dev. $(\mathrm{N} / \mathrm{m})$ & 426 & 451 & 439 \\
\hline
\end{tabular}

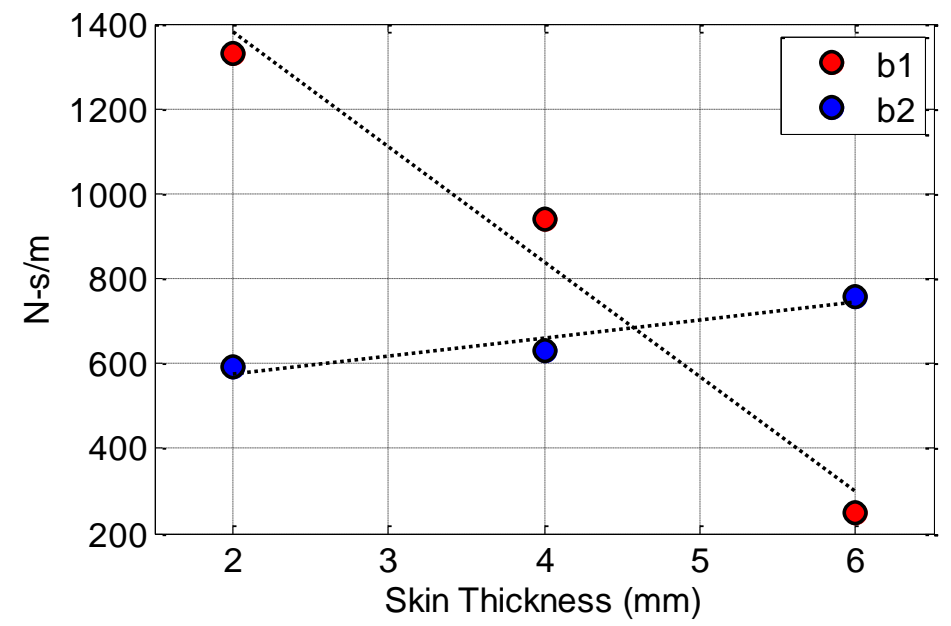

Figure 4-12 Estimated values of $b_{1} \& b_{2}$ for adjusted "dispersion" model for silicone skin

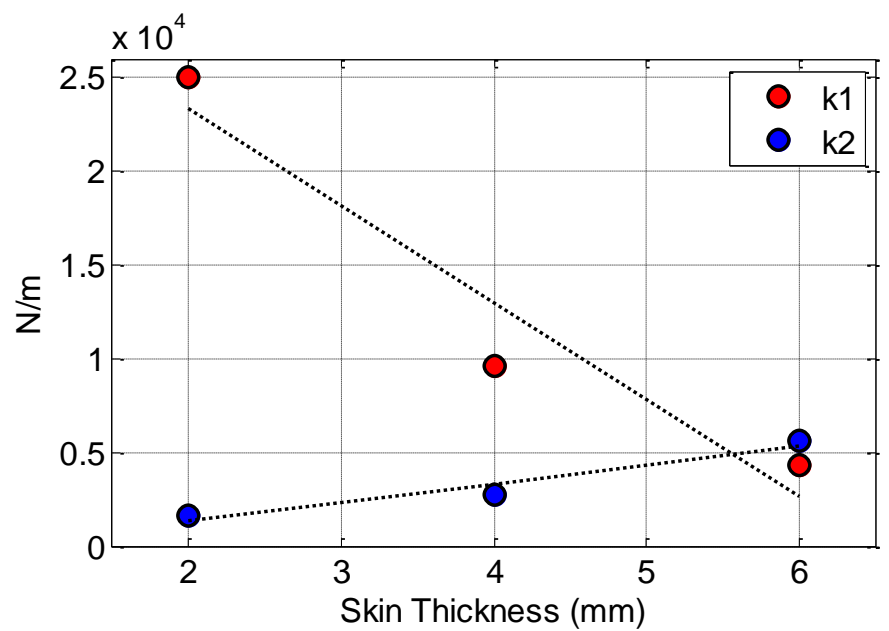

Figure 4-13 Estimated values of $k_{1} \& k_{2}$ for adjusted "dispersion" model for silicone skin 
Parameter identification tests for the adjusted "dispersion" model were also performed on a $4 \mathrm{~mm}$ thick Frubber ${ }^{\circledR}$ skin sample. The same procedure was applied, with a taxel placed underneath the Frubber ${ }^{\circledR}$ skin and a sinusoidal motion input applied on top of the skin. Parameters were identified for three trials on one sample of $4 \mathrm{~mm}$ thick Frubber ${ }^{\circledR}$ skin and are seen in Table 4-6. All four parameters are smaller than the identified parameters for the silicone skin, of any thickness. Also, the standard deviations are lower than for the averages of other individual silicone skin samples. Note, we are only looking at the identified parameters for one sample of Frubber® skin, and not three different samples of the same thickness like for silicone. The reason for this is because it was determined this method of identifying parameters for the "dispersion" model did not relate the applied force and sensed force successfully like the model did for silicone skin. This will be further discussed in section 4.3.2.

Table 4-6 Average values of identified parameters for adjusted "dispersion" for 4mm Frubber® skin

\begin{tabular}{|c|c|}
\hline & 4mm Skin \\
\hline b1 (Ns/m) & 242.6 \\
\hline std. dev. (kg/s) & 3.03 \\
\hline $\mathbf{k 1}(\mathbf{N} / \mathbf{m})$ & 1523 \\
\hline std. dev. (N/m) & 82 \\
\hline b2 (Ns/m) & 243.0 \\
\hline std. dev. (kg/s) & 2.11 \\
\hline $\mathbf{k 2}$ (N/m) & 481 \\
\hline std. dev. (N/m) & 14.5 \\
\hline
\end{tabular}

\subsubsection{Reduced-Order Model Verification}

From each sinusoidal motion parameter identification test we can compare the measured sensed load to the calculated sensed load using the identified parameters and the "dispersion" model. The "dispersion" model we use is the one where the mass has been calculated and only $b_{1}, k_{1}, b_{2}$ and $k_{2}$ were estimated, which we call the adjusted 
"dispersion" model. An example of this on a $6 \mathrm{~mm}$ silicone skin sample is shown in figure 4-14. The error between the measurements and model is largest at the extremums of deformation. A model implementing a nonlinear spring could possibly better match the change in spring constant for varying levels of skin deformation. For our purpose of modeling force transmission in a simulation the current "dispersion" model is satisfactory when using an elastic-like material such as silicone rubber. As seen in figure 4-14 (a graph of the actual sensed force and estimated sensed force using the measurements from the sinusoidal identification test), with a linearly elastic material like silicone rubber the adjusted "dispersion" model closely matches the actual sensed force.

To verify the effectiveness of the "dispersion" model for elastic materials in SkinSim three validation cases were tested. A pseudo-random motion input test was conducted on a $2 \mathrm{~mm}, 4 \mathrm{~mm}$ and $6 \mathrm{~mm}$ robot skin sample. During the tests the load applicator randomly deformed the robot skin samples ranging in depth from $0-1.5 \mathrm{~mm}$ for the $6 \mathrm{~mm}$ sample, $0-1.0 \mathrm{~mm}$ for the $4 \mathrm{~mm}$ sample and $0-0.30 \mathrm{~mm}$ for the $2 \mathrm{~mm}$ sample. The average parameter values for each particular skin sample were implemented offline in the "dispersion" model and compared to the measured sensed force from the pseudorandom motion test. The results can be seen in figure 4-15, 4-16 and 4-17 for a $2 \mathrm{~mm}$, $4 \mathrm{~mm}$ and $5 \mathrm{~mm}$ robot skin sample, respectively. For reference, a sensed force of $5 \mathrm{~N}$ corresponds to a pressure of $70.1 \mathrm{kPa}$ on the taxel. 


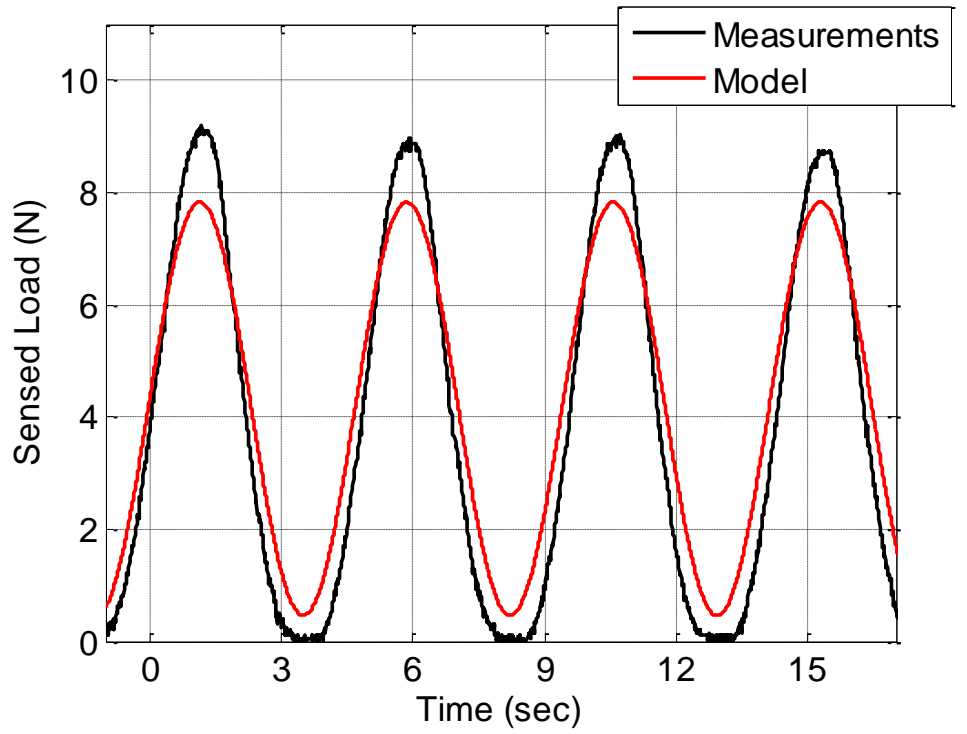

Figure 4-14 Comparison of sensed load between measurements \& "dispersion" model on $6 \mathrm{~mm}$ silicone sample during sinusoidal testing for parameter identification

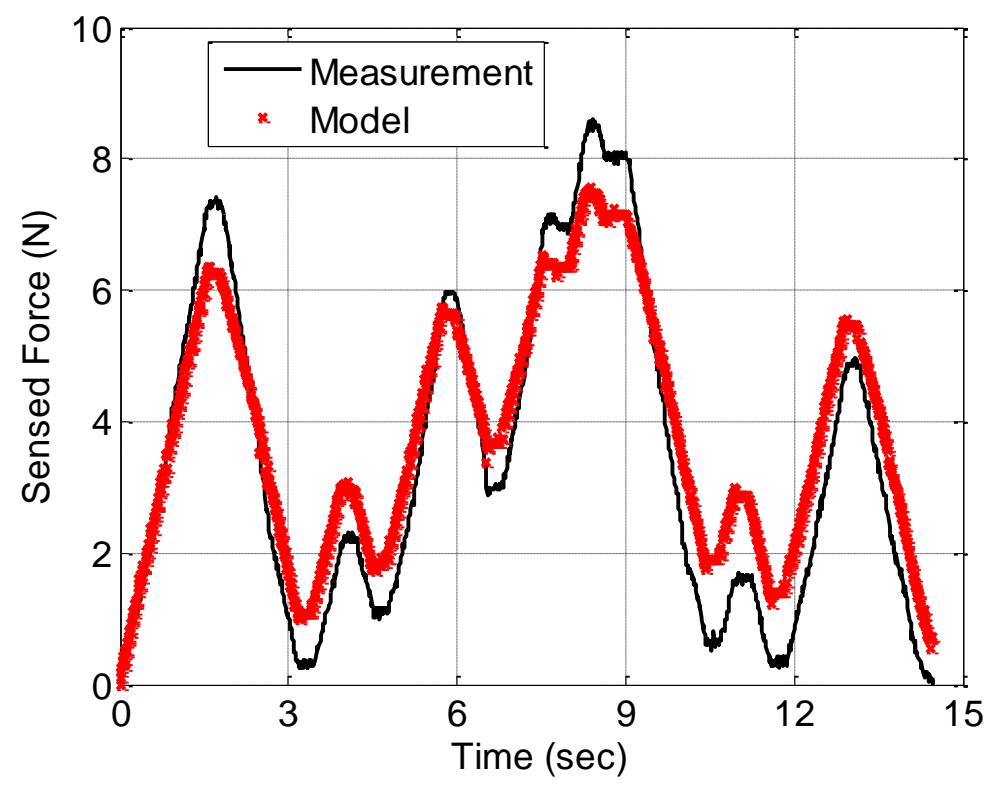

Figure 4-15 Comparison of taxel sensed force \& calculated sensed force using reducedorder "dispersion" model with $2 \mathrm{~mm}$ silicone skin 


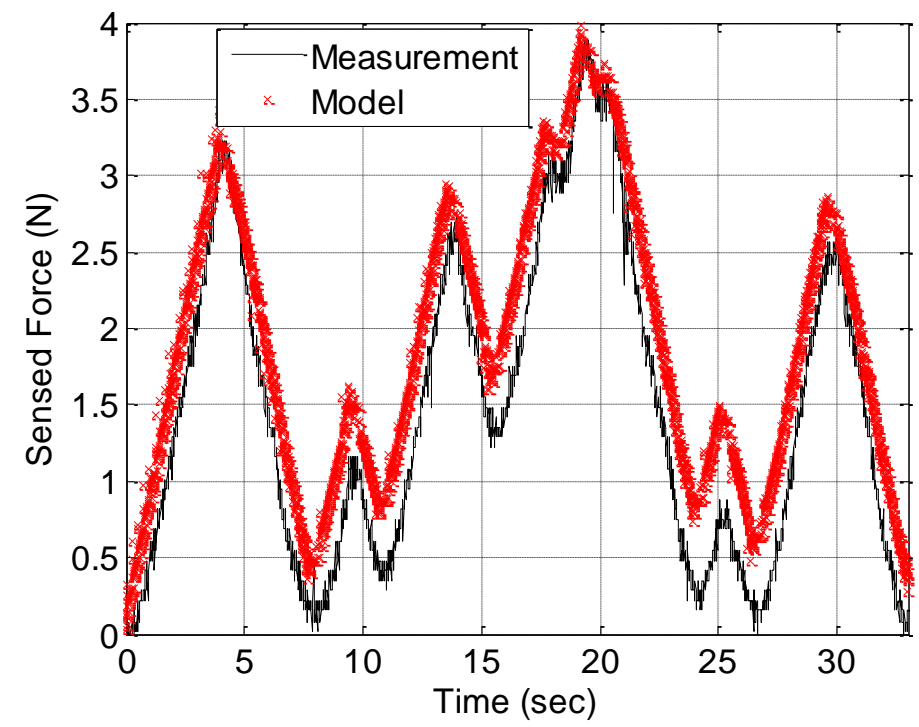

Figure 4-16 Comparison of taxel sensed force \& calculated sensed force using reducedorder "dispersion" model with $4 \mathrm{~mm}$ silicone skin

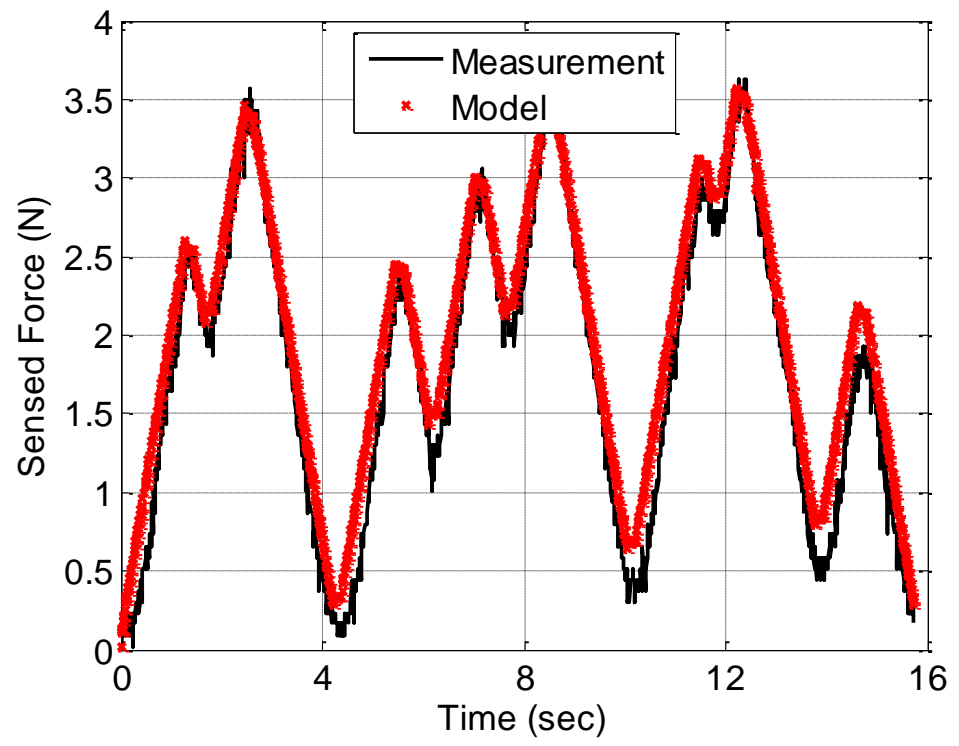

Figure 4-17 Comparison of taxel sensed force \& calculated sensed force using reducedorder "dispersion" model with $5 \mathrm{~mm}$ silicone skin 
The mean squared error (MSE) for each sample is the following: $0.667 \mathrm{~N}$ for the $2 \mathrm{~mm}$ silicone sample; $0.204 \mathrm{~N}$ for the $4 \mathrm{~mm}$ silicone sample; $0.071 \mathrm{~N}$ for the $6 \mathrm{~mm}$ silicone sample; 14.64 for the $4 \mathrm{~mm}$ Frubber ${ }^{\circledR}$ sample. The MSE is less than $8 \%$ of the maximum measured sensed force for each sample of silicone skin. The relative MSE for each is $3.70 \%, 6.28 \%$ and $1.73 \%$ for the $2 \mathrm{~mm}$ silicone, $4 \mathrm{~mm}$ silicone and $6 \mathrm{~mm}$ silicone, respectively. With the silicone skin the "dispersion" model experiences the largest error for the $4 \mathrm{~mm}$ and $6 \mathrm{~mm}$ silicone skin samples when the sensed load is small. The $2 \mathrm{~mm}$ silicone skin sample experiences large error when the sensed load is very small and very large.

The adjusted "dispersion" model was also tested on a very non-linear material, Frubber ${ }^{\circledR}$. The adjusted "dispersion" model parameters were identified for a $4 \mathrm{~mm}$ thick Frubber® skin in the same manner as for each thickness of silicone skin. With elastic skin material, using a LS algorithm to identify parameters for the "dispersion" model works well. However, this is not the case when we perform the same comparison on a Frubber $\AA$ sample. The results of the same test on a $4 \mathrm{~mm}$ Frubber ${ }^{\circledR}$ sample are seen in figure 4-18. The measurements differ greatly from the model estimation. This is because with the LS algorithm we rely upon an assumption of a linear relationship between the force applied and deformation. The LS algorithm uses the sinusoidal deformation as an input; however, as discussed in section 4.1, the relationship between force applied and deformation in Frubber ${ }^{\circledR}$ material is highly nonlinear. 


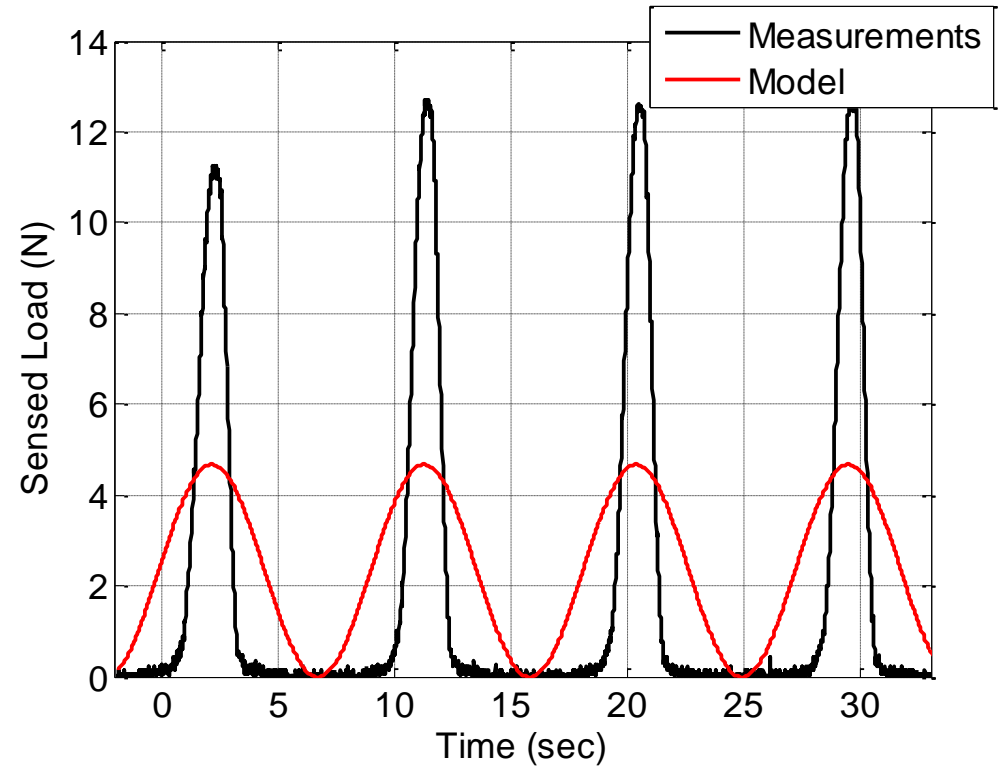

Figure 4-18 Comparison of sensed load between measurements \& "dispersion" model on 4mm Frubber® sample using LS algorithm

Because of the highly nonlinear relationship between force and strain with Frubber ${ }^{\circledR}$ we must approach the parameter identification and force to force relationship with a different method. MATLAB's System Identification Toolbox provides transfer function estimators to relate an input and output through various techniques. We chose a method that fit parameters to a transfer function with prescribed order. However, with this approach we do not use deformation as an input, only the force applied. Through Laplace transformation of equation (27) we can establish a relationship between force applied and force sensed with a transfer function that is linear in the following form:

$$
\frac{\text { Force Sensed }}{\text { Force Applied }}=\frac{b_{1} s+k_{1}}{s^{2}+\left(b_{1}+b_{2}\right) s+\left(k_{1}+k_{2}\right)}
$$

The numerator, which represents the force sensed, only depends on $b_{1}$ and $k_{1}$ while the denominator, which represents force applied, relies on all five "dispersion" model parameters. We expected that the model relating the load cell and sensed force data will 
be considerably more linear than the one relating load cell force and skin displacement. Using transfer function estimation methods of the MATLAB® the model parameters that best relate the force applied to force sensed were identified with measurements conducted on a $4 \mathrm{~mm}$ Frubber ${ }^{\circledR}$ skin sample. Three datasets were analyzed that all came from similar experiments on the same Frubber ${ }^{\circledR}$ sample. All experiments performed sinusoidal deformation on the skin sample. The only difference between the three experiments was the first experiment was performed with a velocity of $0.25 \mathrm{~mm} / \mathrm{sec}$ while experiment 2 and 3 were at $1 \mathrm{~mm} / \mathrm{sec}$. An example of one of the dataset measurements plotting force applied and force sensed can be seen in figure 4-19. The force applied is the model input and the force sensed the output. The identified parameters of the three datasets is seen in Table 4-7. Figure 4-20 shows a comparison of the model fit to the measurements for experiment 1. 


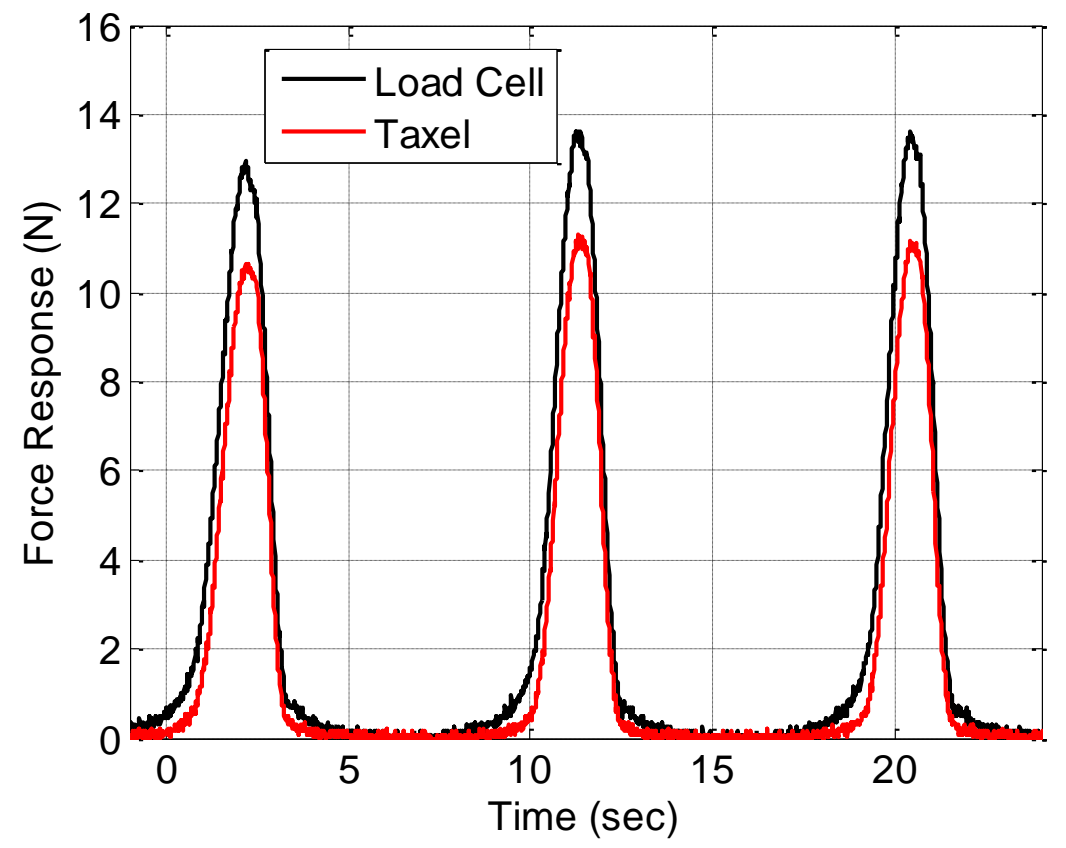

Figure 4-19 Load cell \& taxel response to sinusoidal deformation with 4mm Frubber $\AA$ skin

Table 4-7 Identified parameters for "dispersion" model using MATLAB System Identification Toolbox with 4mm Frubber® skin

\begin{tabular}{|l|c|c|c|c|c|}
\hline & $\begin{array}{c}\text { Deformation } \\
\text { speed }(\mathrm{mm} / \mathrm{s})\end{array}$ & $\begin{array}{c}\frac{b_{1}}{m} \\
(\mathrm{Ns} / \mathrm{kg} / \mathrm{m})\end{array}$ & $\begin{array}{c}\frac{k_{1}}{m} \\
(\mathrm{~N} / \mathrm{m} / \mathrm{kg})\end{array}$ & $\begin{array}{c}\frac{b_{2}}{m} \\
(\mathrm{Ns} / \mathrm{kg} / \mathrm{m})\end{array}$ & $\begin{array}{c}\frac{k_{2}}{m} \\
(\mathrm{~N} / \mathrm{m} / \mathrm{kg})\end{array}$ \\
\hline Experiment 1 & 0.25 & 1.61 & 1.60 & 0.425 & 0.566 \\
\hline Experiment 2 & 1 & 6.69 & 37.1 & 1.88 & 11.3 \\
\hline Experiment 3 & 1 & 6.98 & 39.1 & 1.58 & 9.92 \\
\hline
\end{tabular}




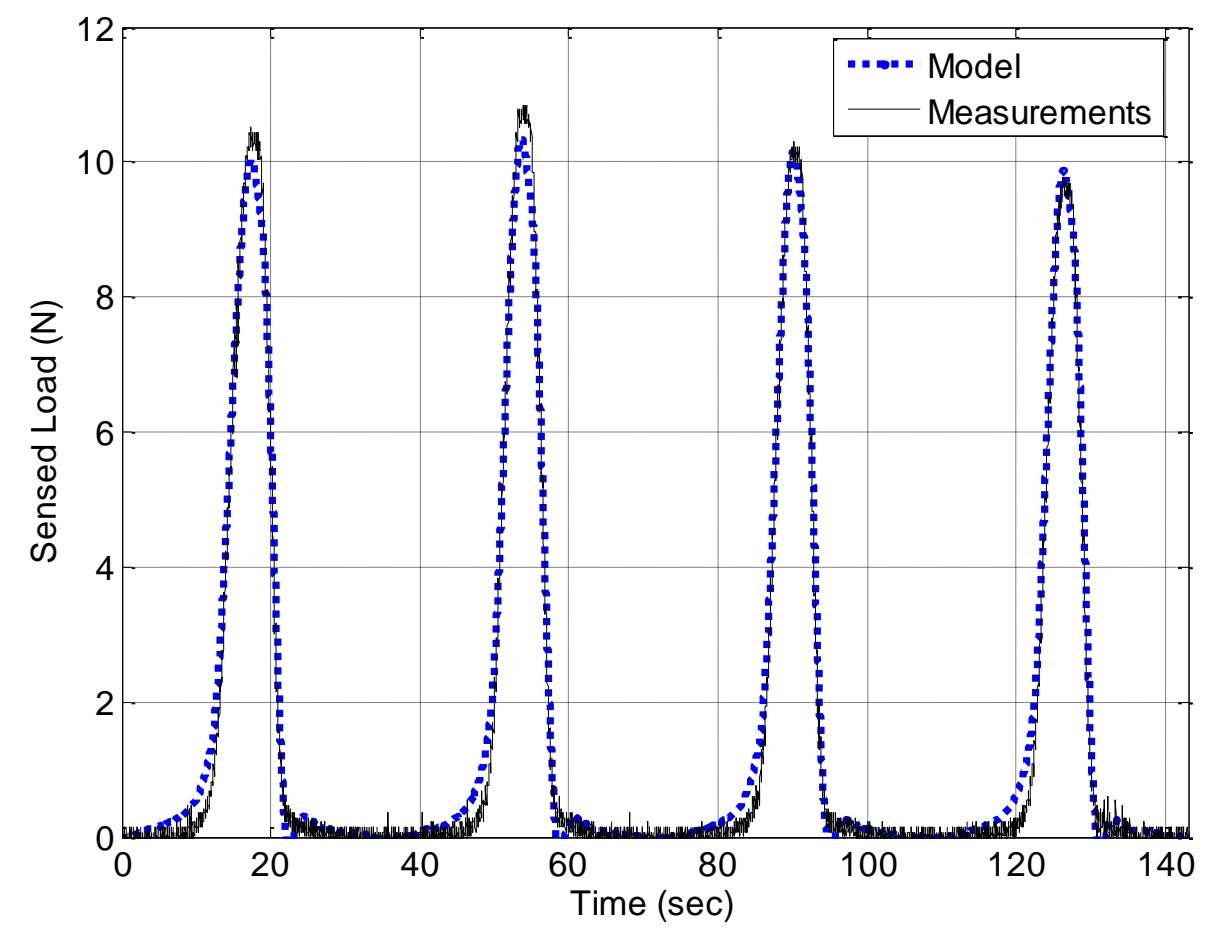

Figure 4-20 Comparison of taxel sensed force \& modeled sensed force using MATLAB System Identification with $4 \mathrm{~mm}$ Frubber ${ }^{\circledR}$ (Experiment 1)

As with the silicone skin samples, we applied a pseudo-random deformation input to the $4 \mathrm{~mm}$ Frubber ${ }^{\circledR}$ to compare the model output to the measured sensed force. The parameters used in the "dispersion" model were the averages of those identified in experiments 1 and 2 . This was because the pseudo-random deformation input was set to a velocity of $1 \mathrm{~mm} / \mathrm{sec}$, which matches the velocity of experiments 1 and 2 . The results of the validation test are seen in figure 4-21. The MSE for the validation was $0.520 \mathrm{~N}$ with a relative MSE of $15.5 \%$. For reference, the average of the relative MSE for the silicone samples with their validation tests was $3.90 \%$. Like with the silicone validation tests, the error is highest at low sensed forces, however, at the peaks (three above $4 \mathrm{~N}$ ) there is low error. 


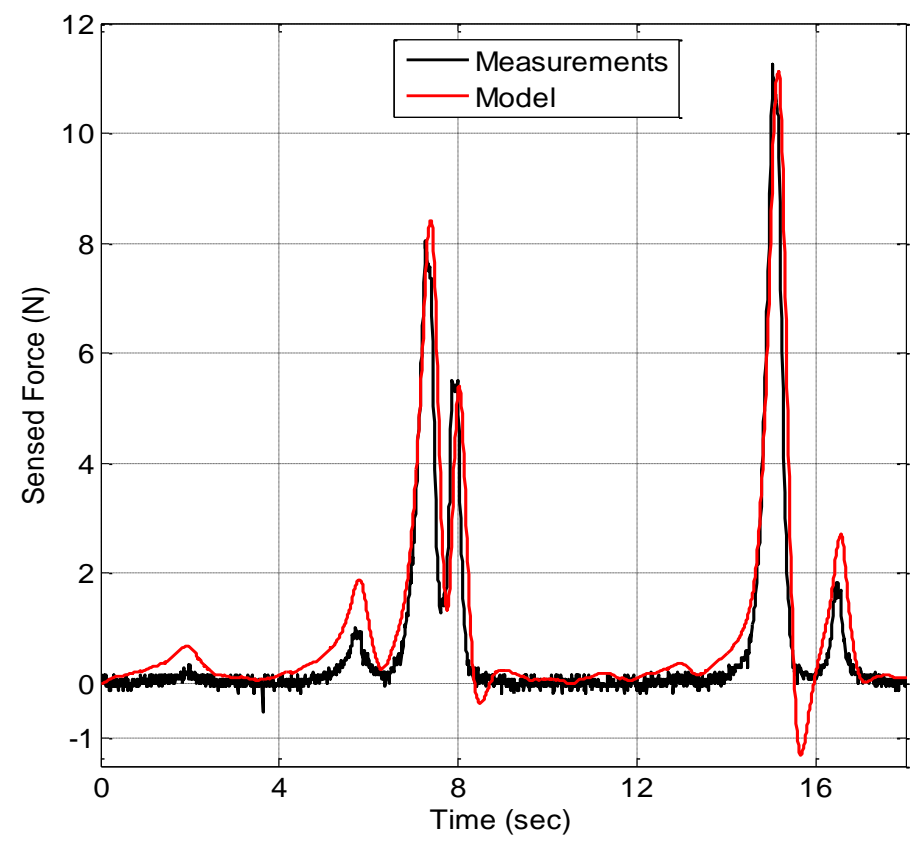

Figure 4-21 Comparison of taxel sensed force \& calculated sensed force for validation test using System Identification with $4 \mathrm{~mm}$ Frubber® skin

\subsubsection{Effect of Soft Skin on Taxel Array}

Tests were performed on a $3 \times 1$ taxel array covered in silicone rubber to determine the effect soft skin has on distributing applied loads to adjacent sensors. A representative top view of the $3 \times 1$ array is shown in figure $4-22$. The red lines indicate the positions the load applicator deformed the robot skin. The taxels were spaced $14 \mathrm{~mm}$ center-to-center underneath a $40 \mathrm{~mm} \times 60 \mathrm{~mm} 4 \mathrm{~mm}$ thick silicone rubber skin sample.

Taxel 1 is centered at position 2, taxel 2 is centered at position 4 and taxel 3 is centered at position 6 . The testbed load applicator was depressed $1.5 \mathrm{~mm}$ into the skin along the length of the taxel array every $7 \mathrm{~mm}$ starting $14 \mathrm{~mm}$ from the center of the first taxel (position \#0). Sensed force taxel measurements from the first second of each depression 
(after reaching maximum deformation) along the array were averaged and can be seen in figure 4-23.

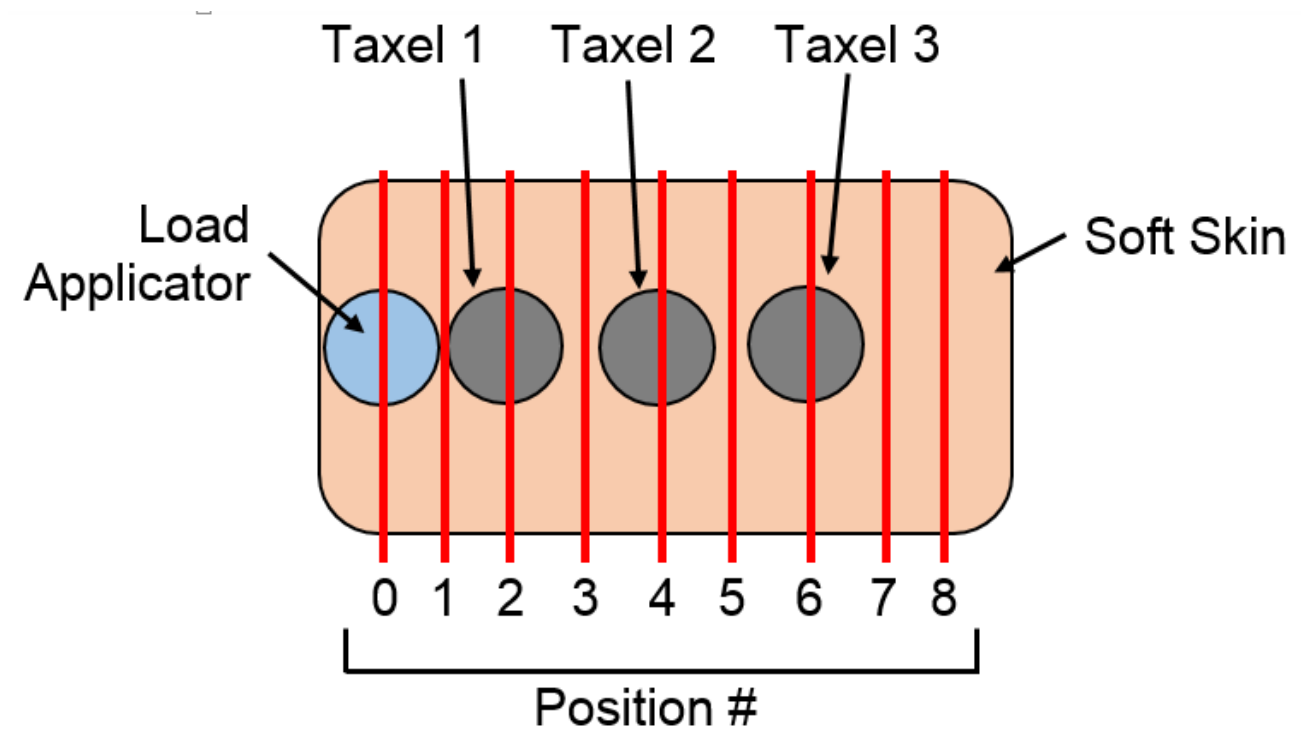

Figure 4-22 Top view of 3x1 taxel array

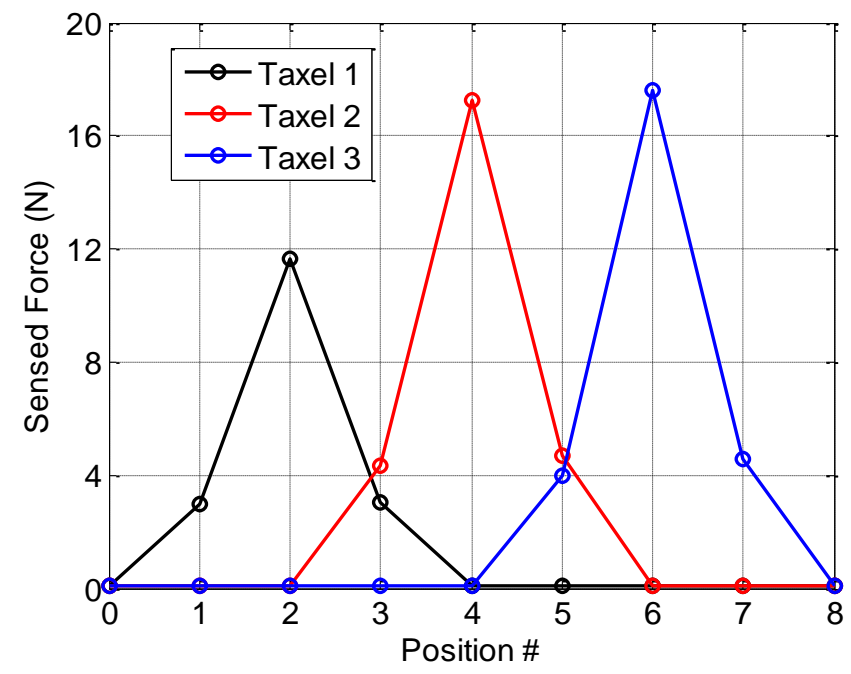

Figure 4-23 Overlapping sensing fields with $3 \times 1$ taxel array

Each taxel recorded the largest sensed force when the deformation was applied to the center of the taxel. Each taxel experienced a similar drop-off in recorded sensed 
force to deformations applied to the positions immediately adjacent to the taxel center. For all taxels zero load was sensed when the deformation occurred greater than one position adjacent to the taxel center. The sensed load at immediately adjacent positions was $74.2 \%$ less than the center load for taxel $1,73.8 \%$ for taxel 2 , and $75.8 \%$ for taxel 3 . From these findings we can infer that the applied load is distributed in a predictable manner to adjacent areas of the robot skin. Additional tests need to be conducted with smaller position increments to better understand the distribution of applied force through a particular skin sample. Also, these type of experiments should be performed on different thicknesses of skin to determine the effect skin thickness has on force distribution. Another insight from this experiment is that the taxels have overlapping sensing fields. For example, both taxel 2 and taxel 3 recorded a sensed load for the deformation that occurred at position \#5. As described in section 2.2.2 of this thesis, overlapping sensing fields can result in hyperacuity (higher spatial acuity than sensor resolution). This type of experiment can help determine the necessary sensor resolution for a desired spatial acuity.

\subsubsection{Taxel Loading Profile with Soft Skin}

In section 4.3.3 we determined that adjacent taxels in an array have overlapping sensing fields. That is, adjacent taxels will both detect a sensed force when a deformation occurs on a point of the skin in between the two taxels. It was also determined that each taxel in the array will have a similar load profile across positions adjacent to the center of the taxel. Because each taxel has a similar load profile, we can "zoom in" on one taxel load profile and use the measurements gained from it to apply standards for every taxel in an array.

To do this, we performed two tests were we placed $4 \mathrm{~mm}$ silicone skin and then $4 \mathrm{~mm}$ Frubber ${ }^{\circledR}$ skin on one taxel, and depressed the load applicator against the skin for 
up to $1 \mathrm{~mm}$ of deformation for the silicone skin, and $3 \mathrm{~mm}$ for the Frubber® skin. We repeated this every $1 \mathrm{~mm}$ moving in a straight line away from the taxel center. Taxel sensed force response measurements were recorded for the entire deformation $(0-1 \mathrm{~mm}$ for silicone skin and 0-3mm for Frubber® skin) of each depression along the straight line. Experiments were performed up to a maximum of $12 \mathrm{~mm}$ away from the taxel center. We would like to note that the measurements of the deformation at position \#2 for the silicone skin were corrupted and not used in this analysis.

To determine the effect of deformation depth on taxel response, the taxel response at deformations of 0 to $1 \mathrm{~mm}$ with $0.10 \mathrm{~mm}$ increments were recorded for silicone skin and then $1 \mathrm{~mm}, 2.5 \mathrm{~mm}, 2.7 \mathrm{~mm}, 2.8 \mathrm{~mm}$ and $2.9 \mathrm{~mm}$ for Frubber ${ }^{\circledR}$ skin. The results can be seen in Table 4-10 and 4-11. The columns in the table represent the distance from the taxel center. The rows in the table represent the taxel sensed force response at different magnitudes of skin deformation. Some of the results are plotted in figure 4-24 and 4-25. It is clear from figure 4-24 that the sensed force magnitudes resemble a Gaussian distribution with silicone skin while with Frubber® skin they seem to be more linear but still somewhat resemble a Gaussian distribution. By assuming measurements on the other side of the taxel are identical to the measurements we obtained we can fit a general Gaussian model for each depth of deformation (i.e., $1 \mathrm{~mm}$, $0.9 \mathrm{~mm}, 0.8 \mathrm{~mm}$, etc. for silicone and $1 \mathrm{~mm}, 2.5 \mathrm{~mm}, 2.7 \mathrm{~mm}$, etc. for Frubber $\left.{ }^{\circledR}\right)$. The general Gaussian equation is as follows:

$$
f(x)=a e^{-\left(\frac{x-b}{c}\right)^{2}}
$$

where $x$ is the position along a straight line that the skin is deformed $(x=12 \mathrm{~mm}$ is taxel center), $a$ is the estimated maximum magnitude of the sensed force at the taxel center, $b$ the position of the taxel center, and $c$ a parameter identifying the shape of the load profile. Using MATLAB each parameter was identified for the given taxel response 
distribution at each depth along with an R-square value determining the fit of the Gaussian model. The results for this are seen in Table 4-8 and 4-9. As expected from figure 4-24 and 4-25, the a value increases with deformation depth and the $b$ value remains the same for both samples of skin. The estimated $c$ values for the silicone sample are very close for deformations greater than $0.40 \mathrm{~mm}$ with an average value of 5.25. The $c$ values being similar could be expected both from the similar shapes of the distributions in figure 4-24 and because the load should be distributed similarly throughout different depths of the same material. The $R^{2}$ value is above 0.99 for deformations greater than $0.40 \mathrm{~mm}$. The high $\mathrm{R}^{2}$ value showcases a Gaussian distribution as a correct fit for these load profiles. Both deformations of $0.20 \mathrm{~mm}$ and $0.30 \mathrm{~mm}$ have $\mathrm{c}$ values close to the larger deformations and an $R^{2}$ value above 0.95 . The $0.10 \mathrm{~mm}$ deformation has a much different $c$ value and a $\mathrm{R}^{2}$ well below that of the others. The smaller deformations are more affected by noise than the larger deformations and therefore the estimated Gaussian model parameters are corrupted. For the Frubber ${ }^{\circledR}$ sample the Gaussian fit also seems appropriate, although less so than for the silicone sample. For the $1 \mathrm{~mm}$ deformation no fit was possible because no measureable load was transferred to the taxel, only noise was present. At the $2.5 \mathrm{~mm}$ deformation and above a noticeable curve begins to develop and the Gaussian fits have an $\mathrm{R}^{2}$ value above .9600 . The shape of the curves (the $b$ value) at and above $2.5 \mathrm{~mm}$ are similar with an average of 4.17. The primary drawback of using a Gaussian fit with the Frubber ${ }^{\circledR}$ skin seems to be the mismatch of the sensed load magnitude at the taxel center. The Gaussian fits for $2.5 \mathrm{~mm}, 2.7 \mathrm{~mm}, 2.8 \mathrm{~mm}$ and $2.9 \mathrm{~mm}$ all have a lower taxel center magnitude (the a value) than the actual magnitude, with an average percent error of $12.6 \%$. 
Table 4-8 Estimated values of general Gaussian model with $4 \mathrm{~mm}$ silicone skin for varying deformation \& $R^{2}$ value for Gaussian fit

\begin{tabular}{|c|c|c|c|c|}
\hline Deformation & $\mathbf{a}$ & $\mathbf{b}$ & $\mathbf{C}$ & $\mathbf{R}^{\mathbf{2}}$ \\
\hline $\mathbf{0 . 1 0 \mathbf { m m }}$ & $0.614 \mathrm{~N}$ & 12 & 6.15 & .7917 \\
\hline $\mathbf{0 . 2 0} \mathbf{m m}$ & $1.56 \mathrm{~N}$ & 12 & 5.41 & .9653 \\
\hline $\mathbf{0 . 3 0 \mathbf { m m }}$ & $2.57 \mathrm{~N}$ & 12 & 5.15 & .9599 \\
\hline $\mathbf{0 . 4 0 \mathbf { m m }}$ & $3.47 \mathrm{~N}$ & 12 & 5.21 & .9903 \\
\hline $\mathbf{0 . 5 0 \mathbf { m m }}$ & $4.54 \mathrm{~N}$ & 12 & 5.19 & .9948 \\
\hline $\mathbf{0 . 6 0 \mathbf { m m }}$ & $5.65 \mathrm{~N}$ & 12 & 5.24 & .9948 \\
\hline $\mathbf{0 . 7 0 m m}$ & $6.86 \mathrm{~N}$ & 12 & 5.28 & .9970 \\
\hline $\mathbf{0 . 8 0} \mathbf{m m}$ & $8.03 \mathrm{~N}$ & 12 & 5.28 & .9966 \\
\hline $\mathbf{0 . 9 0 m m}$ & $9.23 \mathrm{~N}$ & 12 & 5.32 & .9976 \\
\hline $\mathbf{0 . 9 9 m m}$ & $10.5 \mathrm{~N}$ & 12 & 5.25 & .9974 \\
\hline
\end{tabular}

Table 4-9 Estimated values of general Gaussian model with $4 \mathrm{~mm}$ Frubber® skin for varying deformation \& $R^{2}$ value for Gaussian fit

\begin{tabular}{|c|c|c|c|c|}
\hline Deformation & a & b & $\mathbf{C}$ & $\mathbf{R}^{\mathbf{2}}$ \\
\hline $\mathbf{1 . 0 m m}$ & NA & NA & NA & NA \\
\hline $\mathbf{2 . 5 m m}$ & $1.51 \mathrm{~N}$ & 12 & 4.11 & .9642 \\
\hline $\mathbf{2 . 7 m m}$ & $3.66 \mathrm{~N}$ & 12 & 4.08 & .9779 \\
\hline $\mathbf{2 . 8 m m}$ & $4.93 \mathrm{~N}$ & 12 & 4.19 & .9816 \\
\hline $\mathbf{2 . 9 m m}$ & $6.08 \mathrm{~N}$ & 12 & 4.30 & .9861 \\
\hline
\end{tabular}


Table 4-10 Taxel sensed force $(\mathrm{N})$ for varying deformation at given distance from taxel center with $4 \mathrm{~mm}$ silicone skin

\begin{tabular}{|c|c|c|c|c|c|c|c|c|c|c|c|c|}
\hline & Distance & From & Taxel & Center & & & & & & & & \\
\hline Deformation & Omm & $1 \mathrm{~mm}$ & $3 \mathrm{~mm}$ & $4 \mathrm{~mm}$ & $5 \mathrm{~mm}$ & $6 \mathrm{~mm}$ & $7 \mathrm{~mm}$ & $8 \mathrm{~mm}$ & $9 \mathrm{~mm}$ & $10 \mathrm{~mm}$ & $11 \mathrm{~mm}$ & $12 \mathrm{~mm}$ \\
\hline $0.10 \mathrm{~mm}$ & 0.726 & 0.432 & 0.726 & 0.344 & 0.235 & 0.235 & 0.118 & 0.118 & 0.118 & 0.020 & 0.118 & 0.018 \\
\hline $0.20 \mathrm{~mm}$ & 1.63 & 1.43 & 1.24 & 0.922 & 0.628 & 0.334 & 0.235 & 0.235 & 0.235 & 0.020 & 0.235 & 0.020 \\
\hline $0.30 \mathrm{~mm}$ & 2.45 & 2.34 & 2.14 & 1.53 & 0.922 & 0.432 & 0.235 & 0.235 & 0.333 & 0.118 & 0.118 & 0.235 \\
\hline $0.40 \mathrm{~mm}$ & 3.65 & 3.26 & 2.65 & 1.75 & 1.33 & 0.824 & 0.628 & 0.530 & 0.235 & 0.118 & 0.118 & 0.020 \\
\hline $0.50 \mathrm{~mm}$ & 4.36 & 4.36 & 3.45 & 2.55 & 1.75 & 1.04 & 0.726 & 0.333 & 0.333 & 0.235 & 0.118 & 0.118 \\
\hline $0.60 \mathrm{~mm}$ & 5.57 & 5.38 & 4.26 & 3.16 & 2.34 & 1.33 & 0.824 & 0.530 & 0.333 & 0.432 & 0.235 & 0.020 \\
\hline $0.70 \mathrm{~mm}$ & 6.57 & 6.57 & 5.26 & 3.96 & 2.75 & 1.75 & 1.14 & 0.628 & 0.432 & 0.118 & 0.020 & 0.118 \\
\hline $0.80 \mathrm{~mm}$ & 7.79 & 7.79 & 5.96 & 4.67 & 3.16 & 1.94 & 1.33 & 0.824 & 0.726 & 0.333 & 0.235 & 0.118 \\
\hline $0.90 \mathrm{~mm}$ & 9.01 & 8.89 & 6.99 & 5.57 & 3.65 & 2.45 & 1.53 & 0.922 & 0.628 & 0.333 & 0.118 & 0.020 \\
\hline $0.99 \mathrm{~mm}$ & 10.1 & 10.1 & 7.69 & 6.18 & 3.96 & 2.55 & 1.84 & 1.04 & 0.530 & 0.333 & 0.235 & 0.118 \\
\hline
\end{tabular}

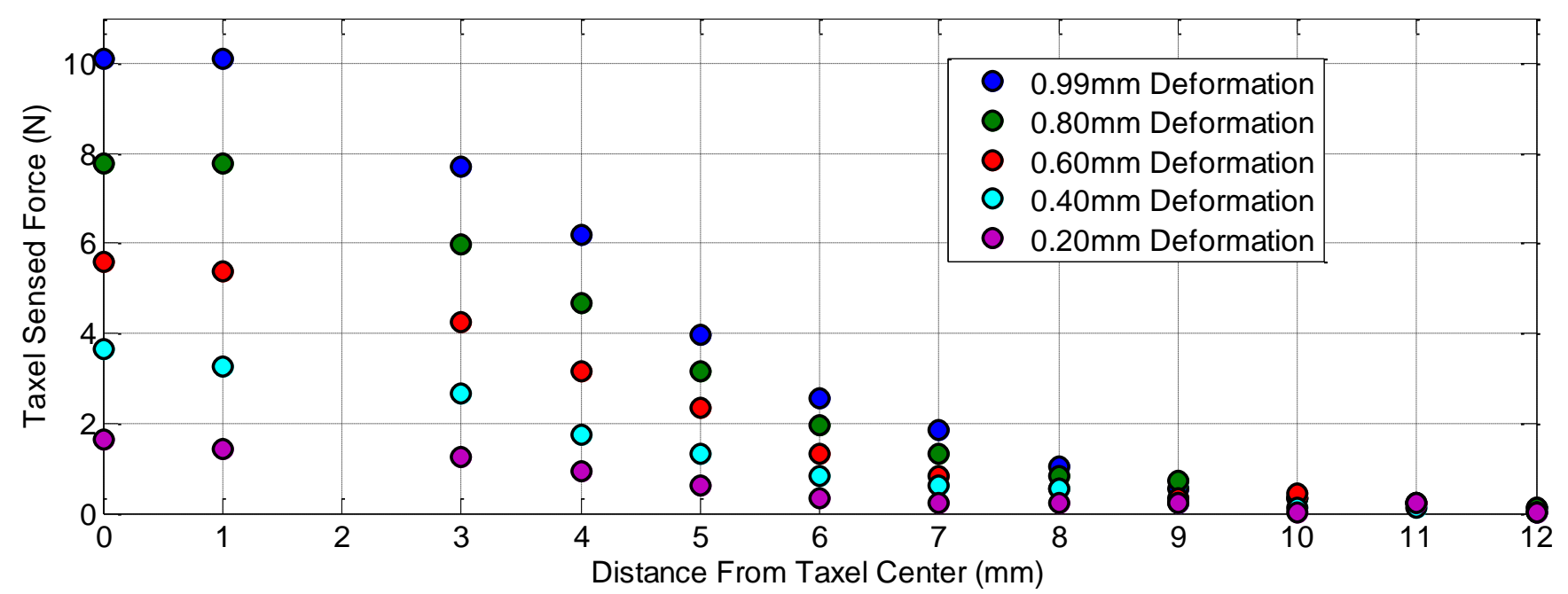

Figure 4-24 Response of taxel to deformation of skin at varying distance from taxel center with $4 \mathrm{~mm}$ silicone 
Table 4-11 Taxel sensed force $(\mathrm{N})$ for varying deformation at given distance from taxel center with 4mm Frubber ${ }^{\circledR}$ skin

\begin{tabular}{|c|c|c|c|c|c|c|c|c|c|c|c|c|}
\hline & Distance & From & Taxel & Center & & & & & & & & \\
\hline Deformation & $0 \mathrm{~mm}$ & $1 \mathrm{~mm}$ & $2 \mathrm{~mm}$ & $3 \mathrm{~mm}$ & $4 \mathrm{~mm}$ & $5 \mathrm{~mm}$ & $6 \mathrm{~mm}$ & $7 \mathrm{~mm}$ & $8 \mathrm{~mm}$ & $9 \mathrm{~mm}$ & $10 \mathrm{~mm}$ & $11 \mathrm{~mm}$ \\
\hline $1.0 \mathrm{~mm}$ & 0.00 & 0.06 & 0.01 & 0.00 & 0.01 & 0.01 & 0.00 & 0.06 & 0.00 & 0.01 & 0.01 & 0.124 \\
\hline $2.5 \mathrm{~mm}$ & 1.63 & 1.43 & 1.24 & 0.922 & 0.628 & 0.334 & 0.235 & 0.235 & 0.235 & 0.020 & 0.235 & 0.020 \\
\hline $2.7 \mathrm{~mm}$ & 2.45 & 2.34 & 2.14 & 1.53 & 0.922 & 0.432 & 0.235 & 0.235 & 0.333 & 0.118 & 0.118 & 0.235 \\
\hline $2.8 \mathrm{~mm}$ & 3.65 & 3.26 & 2.65 & 1.75 & 1.33 & 0.824 & 0.628 & 0.530 & 0.235 & 0.118 & 0.118 & 0.020 \\
\hline $2.9 \mathrm{~mm}$ & 4.36 & 4.36 & 3.45 & 2.55 & 1.75 & 1.04 & 0.726 & 0.333 & 0.333 & 0.235 & 0.118 & 0.118 \\
\hline
\end{tabular}

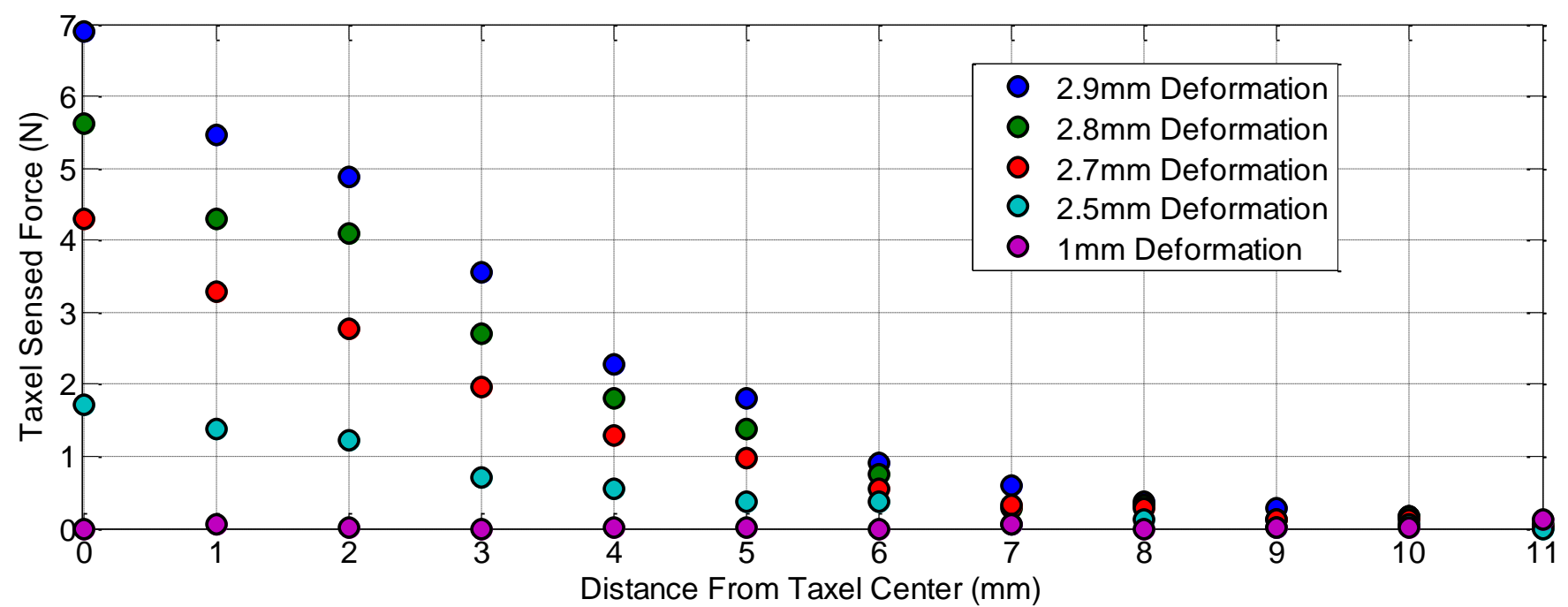

Figure 4-25 Response of taxel to deformation of skin at varying distance from taxel center with $4 \mathrm{~mm}$ Frubber $\circledast$ 
Chapter 5

\section{Conclusion and Future Work}

\subsection{Conclusion}

In this thesis we presented the motivation behind whole-body robot sensing, specifically the importance of tactile sensing for robots. Multiple types of robot skin described in research were presented in addition to the $\mathrm{pHRI}$ simulation environment SkinSim. SkinSim will be used to study density, placement and modality of sensors on social robots. SkinSim requires scalable, "ballpark" approximations relating applied force on robot skin to the sensed force detected by tactile sensors in the skin. Towards this end, a reduced-order model was developed relating the two forces and experiments were conducted to identify parameters for the model utilizing several thicknesses of skin. Validation cases for three skin thicknesses were presented showcasing the effectiveness of the reduced-order "dispersion" model.

To perform these and other robot skin characterization tests an experimental testbed was designed and built. The purpose of the testbed was to provide a platform to test many different types of robot skin. The testbed is a tabletop testing and characterizing apparatus with built in flexibility. The $\mathrm{NI}$ cRIO controller has a chassis that allows multiple modules to be used in addition to the servo drive module and analog input module. The apparatus exceeded all requirements we initially had for it.

The linear actuator has an encoder that provides micrometer resolution which works well with the precise movements performed in our experiments. The encoder can determine the position of the linear stage down to the thousandths of a micrometer. The only drawback is that while the encoder has very good resolution, down to the thousandths of a millimeter, the linear actuator is only able to move reliably down to the tenths of a millimeter. This presented difficulties when zeroing the load applicator on skin 
samples and taxels. For example, at the beginning of all sinusoidal deformation tests, the load applicator z-position was zeroed; however, bringing the linear actuator back to this precise location was extremely difficult. Usually the actuator would come to rest anywhere from $.001 \mathrm{~mm}$ to $.005 \mathrm{~mm}$ from the zeroed location. Because the actuator could only move in $.01 \mathrm{~mm}$ increments (approximately) it required extensive, tedious work to bring the actuator back to the actual zero position. For many experiments, the start position was changed to a position $.001-.002 \mathrm{~mm}$ away from the initial zero position.

Another concern at the beginning of testing was the resolution of the analog input module (NI 9201) which was 12-bit. However, as experiments and model identification have shown, the voltage sensing resolution was not an issue and 12-bit Al modules can successfully be used for this type of work. Additionally, gathering measurements at $100 \mathrm{~Hz}$ was successful. The cRIO can run at much faster sampling rates, however, model identification was successfully implemented using $100 \mathrm{~Hz}$.

An advantage of running the cRIO at $100 \mathrm{~Hz}$ is the guarantee of synchronous measurements. Although the deterministic architecture of the cRIO implies synchronous data measurements, it does not guarantee programs will run lag free. Large measurement sets with VI running multiple complicated loops with several modules in the cRIO could cause the entire program to slow down, possibly resulting in lost measurements or measurements gathered at a slower rate than defined. We believe our work presented in this thesis showcases the viability of running model identification tests at $100 \mathrm{~Hz}$.

Before a taxel type can be used in sensor experiments it must be thoroughly studied. The testbed was used in this thesis to study the FlexiForce A201 force sensor. The A201 is only used as a test case for taxels in soft skin; the testbed is designed to test 
many different types of taxels. The FlexiForce was used because of its cost-effectiveness in testing large-scale sensing applications.

With all taxels utilizing deformable materials we can expect variation between individual taxel responses. We tested the variation in four A201 taxels and confirmed unit-to-unit variation, both in single day tests and multiple day tests. Deformable material taxels must be expected to change throughout continuous testing. Calibration tests performed on the taxel from one day cannot be reliably used for calibrating the taxel on subsequent days. Even within tests performed on the same day the taxel response will change over the course of multiple experiments. To combat this in our testing, taxels were calibrated before each experiment and after, with the average of the two used for post-processing the data. Deformable material taxels will always experience drift and hysteresis, but as shown with the A201 it is possible to neglect these effects if they are minimal. The apparatus we present has the ability to test these non-linear factors for many types of taxels and develop models to correct for them if needed.

We present taxels as they are used embedded in soft elastomer skin. Like the studying of the taxels, the elastomer material used for the soft skin must also be studied before implementation. The apparatus we present can perform necessary experiments to characterize material and develop models to mimic its behavior. As presented, the type of material used in robot skin will determine the type of models that are available for implementation of mimicking its behavior.

We tested both silicone rubber skin and Frubber® skin. The silicone rubber skin is highly elastic and exhibits nearly linear behavior. If a material like silicone rubber is used in robot sensing applications, modeling will be considerably easier than if a material such as Frubber® is used. This is because more rigid and purely elastic materials like silicone rubber have minimal non-linear behavior. Although, as shown through 
experiment, silicone rubber displays hysteresis and drift, it is much less of a factor than with Frubber ${ }^{\circledR}$. A material like Frubber ${ }^{\circledR}$, a porous elastomer, experiences large drift and hysteresis. If the extreme behaviors of Frubber $\AA$ are present in the material selected for robot skin the nonlinear behaviors cannot be ignored. Models exist, however, that can properly mimic the behavior of such non-linear materials and the parameters for those models can be identified through experiments performed on our test apparatus.

Taxel placement in soft robot skin is something that designers will most likely wish to know more about when creating robot skin. Our experiments with taxel placement were limited to the use of the A201 taxels and as such sweeping conclusions are hard to draw. However, what was noticed is that for similar deformations of skin, much larger loads are applied and sensed when the taxel is placed on top of the skin. This is most likely due to the large surface area of the taxel compared to its sensing area. When the taxel is beneath the soft skin the load applicator is only pushing against an area of soft skin equal to that of the area of the load applicator. When the A201 taxel is placed on top of the skin the load applicator is pushing against the rigid taxel, which has an overall area much greater than that of the load applicator surface area (the rigid taxel is acting like a snow-shoe on top of the soft skin). We can conclude that this effect would not be so dramatic if the entire taxel area was the same size as the taxel sensing area (which is not the case for the A201). More tests are needed with different types of taxels to determine a better relationship between taxel placement along the z-direction and expected loads sensed.

Two reduced-order models comparing the applied force on robot skin to the sensed force by taxels embedded underneath soft skin were discussed. Nine different silicone skin samples were tested and "dispersion" model parameters were identified for each. Initially, when the mass of a spring-mass-damper model was considered unknown, 
model parameters did not follow a visible trend. When the "dispersion" model's mass was determined from actual skin properties and held constant model parameters were identified that followed a consistent linear trend.

The "dispersion" model accounts for a load distributed and lost to areas adjacent to the taxel center. As skin thickness increases, a higher percentage of the applied load is lost. It can be gathered from the estimated parameter graphs that at a certain thickness of skin (somewhere between $4 \mathrm{~mm}$ and $6 \mathrm{~mm}$ ) the load sensed becomes less than the load lost. This means more load is distributed to adjacent areas than is picked up by the taxel. This is reflected in the increase of the $k_{2}$ value, which represents the load lost to surroundings. The same trend also presents itself with the damping values $b_{1}$ and $b_{2} ; b_{1}$ decreases and $b_{2}$ increases with an increase of skin thickness.

One might expect the overall spring-constant of the soft skin $\left(k_{1}+k_{2}\right)$ to remain constant as the stiffness of the silicone rubber does not change with thickness, but that is not reflected in our findings; instead, the overall spring-constant continually decreases, driven primarily by the decrease in $k_{1}$. This could be because the adjusted "dispersion" model still does not accurately reflect physical values, despite consistent trends in the parameters being presented. Although the adjusted model most likely does not reflect real-world physical values, it still can be used for SkinSim simulation.

Because the trends in the estimated values for the adjusted "dispersion" model are approximately linear, users of SkinSim can implement model parameters for a range of skin thicknesses and are not limited to the three thicknesses tested here. In addition, because the model verifications tests were consistent in their relative accuracy, trust can be placed in the identified parameters for use in large-scale sensor experiments for simulation. 
These conclusions are only for robot skin implementing a linear elastomer such as silicone rubber with parameters identified through LS. It was shown that for a highly nonlinear material such as Frubber ${ }^{\circledR}$ using a linear LS algorithm to identify parameters for the "dispersion" model is inadequate. This is because the Frubber® stress vs. strain relationship is better modeled by a nonlinear spring and also that the stress is greatly affected by the time history of deformation.

To accurately model the force to force relationship in a material like Frubber® we focused on identifying a transfer function that strictly relates force applied to force sensed in the robot skin. This was done by using Laplace transform techniques to acquire a transfer function relating an input force applied to an output force sensed. Using MATLAB's System Identification Toolbox parameters were identified for the transfer function model.

The three experiments presented for System Identification used two different deformation velocities. This affected the identified parameters, resulting in smaller parameters when the experiment deformation ran at a slower speed. This illuminates the effect strain rate has on a material like Frubber ${ }^{\circledR}$ even when considering only a force to force relationship. It is possible that adding a nonlinear or memory term to the model could result in the parameters remaining the same despite a change in strain rate. This needs to be further studied because during real pHRI deformation to robot skin will be both unknown and changing.

When validating the parameters through a pseudo-random deformation input experiment we found the model was able to accurately mimic actual sensed force measurements. However, the relative MSE was higher than with the silicone skin (an average of $3.9 \%$ for silicone skin compared to $15.5 \%$ for Frubber $\AA$ ). 
A 3x1 taxel array was constructed to study the dispersion of applied load throughout a skin sample. A test such as this can help define the limits of taxel spatial acuity relative to taxel resolution. Because of overlapping sensing fields from adjacent taxels the position of a load applied at an intermediate point between two taxels can be narrowed down to a certain range better than only using the response form one taxel.

Overlapping sensing fields can be expected to be present in most taxel arrays embedded or underneath soft elastomer skin. This is because the elastomer skin distributes the applied load throughout the skin, and not just to the area directly underneath the contact point. Through the $3 \times 1$ taxel array tests it is confirmed this behavior can be studied with our testing apparatus.

The tests also demonstrate how load distribution for a given taxel will be similar to all taxels in the array (same thickness of skin, same load applied, etc.). Due to this, studying the response of one taxel and its response to loads applied at adjacent points to its center can be used to characterize all taxels in a given array. This was performed on an A201 in 4mm thickness silicone and 4mm Frubber ${ }^{\circledR}$ skin by applying a consistent deformation at $1 \mathrm{~mm}$ increments in a straight line away from the taxel center. The response of the taxel was compared at equivalent deformations for each increment away from the taxel center.

The load profile for each thickness of skin is easily identifiable as a Gaussian distribution and using model identification techniques in MATLAB the parameters for each general Gaussian model can be found. Through these tests it was determined that the load profile for each level of deformation keeps a similar Gaussian "shape" which we might expect from the nearly linear silicone rubber material. We can assume that this consistency in shape will present itself for all thicknesses of silicone skin. Because of this, it is possible only one level of deformation need be tested for each thickness of skin - 
probably the maximum deformation expected. This can be done because with one set of deformation tests (only one level of deformation done in increments along a straight line from the taxel center) the "shape" of the Gaussian distribution can be determined. From there, the only additional change needed in the Gaussian model is the load sensed for a particular desired deformation centered at the taxel. The sensed loads at distances in a straight line from the taxel center can then be estimated because all parameters in the model are known (the "shape" of the distribution, the maximum magnitude of the sensed load at the taxel center, and the distance from the center of the taxel for the desired sensed load). Also, the Gaussian load profile distribution coupled with the adjusted "dispersion" model will be used in SkinSim to simulate real world interactions.

With knowledge of the tests described in this thesis as well as the apparatus to perform all needed experiments, many different types of taxel and skin combinations can be characterized, modeled and used in simulation (SkinSim). While not all cases and types of robot skin are addressed in this thesis, a groundwork of robot skin modeling has been established along with a platform to perform many of the tests that will be required in the future.

\subsection{Future Work}

Future research with the experimental testbed presented in this thesis should include testing and characterizing additional types of skin sensors, including PPS sensors housed at UTARI. For this thesis, only a flat, cylindrical load applicator was used, but in the future it will be useful to determine how taxels react to more human like contacts, such as a solid member covered in soft skin (like a human finger). Additionally, linear servos should replace the manual $x-y$ stages currently used as a base for skin samples. This will improve the speed, accuracy and repeatability for testing taxel arrays as well as defining sensing-fields centered on a taxel. 
An incremental force-controller was developed but no experiments were conducted on robot skin samples with this controller. Instead, a position-based explicit force controller should be programmed in LV to use when testing skin samples. Programs utilizing a position-based explicit force controller would be able to apply desired loading profiles based on force, instead of position.

Modeling non-linear behavior of elastomer skin needs to be further studied. Once the reduced-order model is implemented and tested in SkinSim, the users will have a better indication of what types of models can be used in future iterations. More computationally expensive nonlinear models could be more necessary than currently thought. However, this will not be known until SkinSim is functional and can be extensively tested. 
References

[1] C. C. Kemp, A. Edsinger and E. Torres-Jara, "Challenges for Robot Manipulation in Human Environments," IEEE Robotics and Automation, vol. 14, no. 1, pp. 20-29, 2007.

[2] D. O. Popa, "Multi-Modal Skin and Garments for Healthcare and Home Robots," National Robotics Initiative, USA, 2012.

[3] R. S. Dahiya, G. Metta, M. Valle and G. Sandini, "Tactile Sensing - From Humans to Humanoids," IEEE Transactions on Robotics, vol. 26, no. 1, 2010.

[4] A. Habib, I. Ranatunga, K. Shook and D. O. Popa, "SkinSim: A Simulation Environment for Multimodal Robot Skin," in under review for IEEE International Conference on Automation Science and Engineering, Taipei, Taiwan, August, 2014.

[5] F. Ikhouane and J. Rodellar, Systems with Hysterisis - Analysis, Identification and Control using the Bouc-Wen Model, West Sussex, England: John Wiley \& Sons Ltd, 2007.

[6] N. Instruments, "NI LabVIEW for CompactRIO Developer's Guide," 2013.

[7] D. Hanson, "Expanding the Aesthetics Possibilitiesfor Humanlike Robots," in IEEE Humanoid Robotics Conference, special session on the Uncanny Valley, Tskuba, Japan, 2005.

[8] H. Yamada, Strength of Biological Materials, Huntington, New York: Robert E. Krieger Publishing Company, 1973.

[9] R. S. Johansson and A. B. Vallbo, "Tactile Sensibility in the Human Hand: Relative and Absolute Densities of Four Types of Mechanoreceptive Units in Glabrous Skin," J. Physio., vol. 286, pp. 283-300, 1979. 
[10] K. Myles and M. S. Binseel, "The Tactile Modality: A Review of Tactile Sensitivity and Human Tactile Interfaces," Army Research Laboratory, May, 2007.

[11] M. S. and S. H., "Thermotactile Perception Thresholds Measurement Conditions," Ind Health, Oct. 2002.

[12] R. L. Klatzky and S. J. Lederman, "Touch," Experimental Psyhology (Handbook of Psychology Series), vol. 4, pp. 147-176, 2003.

[13] J. M. Wolfe, K. R. Kluender, D. M. Levi, L. M. Bartoshuk, R. S. Herz, R. L. Klatzky and S. J. Lederman, Sensation and Perception, Sunderland, MA: Sinauer, 2006.

[14] E. R. Kandel, J. J. Schwartz and T. M. Jessel, Principles of Neural Science, 4th ed., New York: McGraw-Hill Medical, 2000.

[15] M. Cutkosky, R. Howe and W. Provancher, "Ch. 9: Force and Tactile Sensors," in Springer Handbook of Robotics, Springer Handbooks, 2008.

[16] R. D. Howe, "Tactile Sensing and Control of Robotic Manipulation," Journal of Advanced Robotics, vol. 8, pp. 245-261, 1994.

[17] V. J. Lumelsky, M. S. Shur and S. Wagner, "Sensitive Skin," in IEEE Sensors, vol. 1, pp. 41-51, 2001.

[18] R. S. Dahiya, "Towards Ultra-Thin High-Performance Bendable Electronic Skin," in ICRA 2013: Research Frontiers in Electronic Skin Technology, Karlsruhe, Germany, 2013.

[19] R. I. B. K. Bock, "MultiFunctional System Integration in Flexible Substrates," in ICRA 2013 - Frontiers in , 2013.

[20] P. Mittendorfer and G. Cheng, "From a Multi-Modal Intelligent Cell to a SelfOrganizing Robotic-Skin," in ICRA 2013, 2013.

[21] P. Mittendorfer and G. Cheng, "Humanoid Multimodal Tactile-Sensing Modules," IEEE Transactions on Robotics, vol. 27, no. 3, pp. 401-410, June 2011. 
[22] A. Schmitz, P. Maiolino, M. Maggiali, L. Natale, G. Cannata and G. Metta, "Methods and Technologies for the Implementation of Large-Scale Robot Tactile Sensors," IEEE Transactions on Robotics, vol. 27, no. 3, pp. 389-400, June 2011.

[23] A. Schmitz, P. Maiolino, M. Maggiali, L. Natale, G. Cannata and G. Metta, "Methods and Technologies for the Implementation of Large-Scale Robot Tactile Sensors," IEEE Transactions on Robotics, vol. 27, no. 3, pp. 389-400, June 2011.

[24] M. W. Strohmayr, H. Worn and G. Hirzinger, "The DLR Artificial Skin, Step I: Uniting Sensitivity and Collision Tolerance," in ICRA 2013, Karlsruhe, Germany, May 2013.

[25] M. W. Strohmayr and D. Schneider, "The DLR Artificial Skin, Step II: Scalability as a Prerequisite for Whole-Body Covers," in IROS IEEE/RSJ IROS, Tokyo, Japan, November, 2013.

[26] Y. Tenzer, L. P. Jentoft and R. D. Howe, "Inexpensive and Easily Customized Tactile Array Sensors using MEMS Barometers Chips," Harvard School of Engineering and Applied Sciences, 2012.

[27] N. F. Lepora, U. Martinez-Hernandez, M. Evans, L. Natale, G. Metta and T. J. Prescott, "Embodied Hyperacuity for Robot Touch," in ICRA 2013: Research Frontiers in Electronic Skin Technology, Karlsruhe, Germany, 2013.

[28] M. Ohka, A. Tsunoai, T. Kayaba and H. Yussof, "Skin Function of Optical Three-Axis Tactile Sensor," in ICRA 2013: Research Frontiers in Electronic Skin Technology, Karlsruhe, Germany, 2013.

[29] K. Bock, R. I. Bose, G. Klink, C. Landesberger, A. Ohlander and E. Yacoub-George, "Multifunctional System Integration in Flexible Substrates," in ICRA 2013: Research Frontiers in Electronic Skin Technology, Karlsruhe, Germany, 2013. 
[30] V. Vinciguerra, S. Smerzi, E. Spoto, A. Scuderi, M. Renna and L. G. Occhipinti, "Flexible and Conformable Smart Sensor Systems From Concept and Integration to Multiple Sector Applications," in ICRA 2013: Research Frontiers in Electronic Skin Technology, Karlsruhe, Germany, 2013.

[31] L. Maiolo, A. Pecora, F. Maita, A. Minotti, G. Fortunato, D. Ricci and G. Metta, "Enhanced Piezoelectric Hybrid Tactile Sensors Fully Integrated on Ultra-Thin Polyimide Substrates for Robotic Applications," in ICRA 2013: Research Frontiers in Electronic Skin Technology, Karlsruhe, Germany, 2013.

[32] D. Hanson and V. White, "Converging the Capabilities of ElectroActive Polymer Artificial Muscles and the Requirements of Bio-inspired Robotics," in Proc. SPIE, Electroactive Polymer Actuators and Devices Conf., 10th Smart Structures and Materials Symposium, San Diego, USA, 2004.

[33] R. Lakes, Viscoelastic Materials, New York, NY: Cambridge University Press, 2009.

[34] W. N. Findley, J. S. Lai and K. Onaran, Creep and Relaxation of Nonlinear Viscoelastic Materials, Amsterdam, New York: North-Holland Publishing Company, 1976.

[35] A. D. Drozdov, Viscoelastic Structures, Mechanics of Growth and Aging, San Diego, CA: Academic Press, 1998.

[36] J. Meyer, "Textile Pressure Sensor: Design, Error Modeling and Evaluation," ETH Zurich University, Zurich, Switzerland, 2008.

[37] J. L. Crassidis and J. L. Junkins, Optimal Estimation of Dynamic Systems, 2nd Ed., Boca Raton, FL: CRC Press, 2012.

[38] National Instruments, "Real-Time CompactRIO," 1903 2014. [Online]. Available: http://sine.ni.com/nips/cds/view/p/lang/en/nid/203964. 
[39] National Instruments, "I/O Modules, NI 9201," [Online]. Available:

http://sine.ni.com/nips/cds/view/p/lang/en/nid/14588. [Accessed 1903 2014].

[40] National Instruments, "I/O Modules, NI 9516," [Online]. Available:

http://sine.ni.com/nips/cds/view/p/lang/en/nid/206354. [Accessed 1903 2014].

[41] M. Motor, "Maxon Motor Control, Operating Instructions," [Online]. Available:

ftp://apollo.ssl.berkeley.edu/pub/Pointing_Studies/Ballon\%20Pointing/Maxon\%20

Servo\%20Amp\%20Manual.pdf. [Accessed 1903 2014].

[42] B. Komati, M. R. Pac, I. Ranatunga, C. Clevy, D. O. Popa and P. Lutz, "Explicit Force Control vs. Impedance Control for Micromanipulation," in ASME 2013 International Design Engineering Technical Conference \& Computers and Information in Engineering Conference, Portland, OR, August 2013.

[43] Tekscan, "FlexiForce Sensors," [Online]. Available: http://www.tekscan.com/flexibleforce-sensors. [Accessed 1903 2014].

[44] Phidgets, "1120 User Guide," [Online]. Available: http://www.phidgets.com/docs/1120_User_Guide. [Accessed 1903 2014].

[45] Phidgets, "1120_0 - FlexiForce Adapter," [Online]. Available: http://www.phidgets.com/products.php?product_id=1120. [Accessed 1903 2014].

[46] T. Techniques, "Mini Low Porfile Load Cell Universal / Tension or Compression," [Online]. Available: http://www.transducertechniques.com/mlp-load-cell.aspx. [Accessed 1903 2014].

[47] DSM, "Product Data Sheet: Somos(R) WaterShed XC11122," 2012. [Online]. Available: http://www.dsm.com/products/somos/en_US/offerings/offeringssomos-water-shed.html\#. [Accessed 2003 2014]. 
[48] P. M. Sain, M. K. Sain and B. F. Spencer, "Models for Hysteresis and Application to Structural Control," in Proceedings of the American Control Conference, Albuquerque, NM, June 1997. 


\section{Biographical Information}

Kyle R. Shook grew up in Carrollton, Texas and has resided in North Texas for the majority of his life. He earned both his B.S. and M.S. in Mechanical Engineering from The University of Texas at Arlington in 2011 and 2014, respectively. From May 2011 to May 2013 Kyle worked at the UTA Research Institute (UTARI) on multiple design projects including the biomedical device project Biomask. Kyle has always had a desire to help those who have difficulty helping themselves and hopes to improve the lives of people with disabilities. Kyle's main research interests are in biomedical device technology and robot automation and control. One day he will dunk on a regulation size basketball hoop. 